\title{
Prevention of long-term sickness absence and major depression through early intervention
}

Citation for published version (APA):

Lexis, M. A. S. (2011). Prevention of long-term sickness absence and major depression through early intervention. [Doctoral Thesis, Maastricht University]. Maastricht University. https://doi.org/10.26481/dis.20110520ml

Document status and date:

Published: 01/01/2011

DOI:

$10.26481 /$ dis.20110520ml

Document Version:

Publisher's PDF, also known as Version of record

\section{Please check the document version of this publication:}

- A submitted manuscript is the version of the article upon submission and before peer-review. There can be important differences between the submitted version and the official published version of record. People interested in the research are advised to contact the author for the final version of the publication, or visit the DOI to the publisher's website.

- The final author version and the galley proof are versions of the publication after peer review.

- The final published version features the final layout of the paper including the volume, issue and page numbers.

Link to publication

\footnotetext{
General rights rights.

- You may freely distribute the URL identifying the publication in the public portal. please follow below link for the End User Agreement:

www.umlib.nl/taverne-license

Take down policy

If you believe that this document breaches copyright please contact us at:

repository@maastrichtuniversity.nl

providing details and we will investigate your claim.
}

Copyright and moral rights for the publications made accessible in the public portal are retained by the authors and/or other copyright owners and it is a condition of accessing publications that users recognise and abide by the legal requirements associated with these

- Users may download and print one copy of any publication from the public portal for the purpose of private study or research.

- You may not further distribute the material or use it for any profit-making activity or commercial gain

If the publication is distributed under the terms of Article $25 \mathrm{fa}$ of the Dutch Copyright Act, indicated by the "Taverne" license above, 
Prevention of long-term sickness absence and major depression through early intervention 
Prevention of long-term sickness absence and major depression through early intervention

Monique Agnes Servaas Lexis

ISBN 978-90-9026115-7

Lay-out : Jos Bruystens, Maastricht

Cover : Jos Bruystens, Maastricht

Printed by : Wilco, Amersfoort

(C) 2011, M.A.S. Lexis

All rights reserved. No part of this thesis may be reproduced or transmitted in any form or by any means, electronic or mechanical, including photocopying, recording or any information storage or retrieval system, without permission in writing from the author, or, when appropriate, from the publishers of the publications. 


\title{
Prevention of long-term sickness absence and major depression through early intervention
}

\author{
PROEFSCHRIFT
}

ter verkrijging van de graad van doctor

aan de Universiteit Maastricht,

op gezag van de Rector Magnificus,

Prof. mr. G.P.M.F. Mols,

volgens het besluit van het College van Decanen,

in het openbaar te verdedigen

op vrijdag 20 mei 2011 om 14.00 uur

door

Monique Agnes Servaas Lexis 


\section{Promotores}

Prof. dr. IJ. Kant

Prof. dr. ir. P.A. van den Brandt

\section{Copromotor}

Dr. N.W.H. Jansen

\section{Beoordelingscommissie}

Prof. dr. R.A. de Bie (voorzitter)

Prof. dr. A. Arntz

Prof. dr. A.J. van der Beek (EMGO instituut, VU Medisch Centrum, Amsterdam)

Prof. dr. F.J.N. Nijhuis

Dr. C. van Vliet (Directeur Kwaliteitsbureau NVAB, Utrecht)

This project was funded by the Netherlands Organization of Health Research and Development (Zorg Onderzoek Nederland, grant no. 62200024), and by the Occupational Health Services Beter, Amsterdam, the Netherlands.

The studies presented in this thesis were conducted at the Department of Epidemiology (Maastricht University), and were embedded in CAPHRI School for Public Health and Primary Care. CAPHRI is part of the Netherlands School of Primary Care Research (CaRe), which has been acknowledged since 1995 by the Royal Netherlands Academy of Arts and Sciences (KNAW). 
Nor pap, mam en Chris 



\section{Contents}

1 General introduction 9

2 Depressive complaints as a predictor of sickness absence among the working population

3 Prediction of long-term sickness absence among employees with depressive complaints

$4 \quad$ Experience of health complaints and help seeking behavior in employees screened for depressive complaints and risk of future sickness absence

5 Prevention of long-term sickness absence and major depression in high risk employees:

a randomized controlled trial

6 The relationship between protocol adherence and the efficacy of a preventive intervention aimed at the prevention of long-term sickness absence and major depression

7 General discussion

Summary

Samenvatting

Dankwoord

About the author 



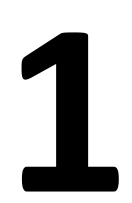

General introduction 



\section{Long-term sickness absence}

Sickness absence, and in particular long-term sickness absence, constitutes a major public health and economical problem in Western societies. Long-term sickness absence has many consequences, for employees, their families, for work-places and for society. ${ }^{1}$ These consequences encompass healthrelated, social and financial issues. ${ }^{2-4}$ For the employee, sickness absence can be the beginning of job dismissal and it may cause social impairment, such as isolation and inactivity. This in turn, can result in for example depressed mood, lower self confidence and may lead to an increase in health care use. ${ }^{4,5}$ For employers and society, long-term sickness absence mainly has financial consequences, resulting in costs due to loss of man power, payments for temporary workers, reduced productivity, increased job turnover, disability benefits and rehabilitation programs..$^{5,6}$

The magnitude of the problem of long-term sickness absence has been acknowledged by many Western countries and has led to development of legislation on sickness absence and has made sickness absence one of the top priorities of the governments of European Union countries. ${ }^{7}$ From 1994 until 2009, sickness absence prevalence in the Netherlands has decreased from approximately $6 \%$ to 4.3\%, due to introduction of the Sickness Absence Reduction Act in $1994 .^{8}$ However, compared with other European countries, sickness absence in the Netherlands is relatively high. ${ }^{9}$ Moreover, despite this reduction, sickness absence still remains a major problem, in terms of costs, labor participation and social consequences. Although long-term sickness absence constitutes only a small fraction of absence episodes, longer absences contribute considerably to the total days lost and it is the rather small population of employees with long-term sickness absences who account for the majority of costs. In the Dutch economy there are about 7.5 million workers. ${ }^{10}$ In 2009, Dutch employers paid a total of approximately 7.5 billion Euros of direct costs to employees due to sickness absence, indirect costs for example due to reduced labor participation are not included yet. ${ }^{11}$ Furthermore, longterm sickness absence is known to be a preliminary stage of work disability. ${ }^{2,12,13}$ The proportion of persons of the Dutch working-age population on incapacity benefits was approximately $10 \%$ in 2009. ${ }^{11}$ As a result, the total annual costs of sickness absence and work disability in the Netherlands were still more than 20 billion Euros in 2009. ${ }^{11}$ Similar figures can be quoted for other European high-income countries. ${ }^{14,15}$

The two most common causes of long-term sickness absence are muskulo-skeletal complaints and mental health complaints. Together, muskulo-skeletal complaints, such as chronic aspecific low back pain and complaints of arm, neck and/or shoulder (CANS), and mental health complaints, such as depression and burnout, account for over $70 \%$ of the certified sick leave in the Netherlands. ${ }^{16}$ In most high-income countries mental health complaints now are the leading cause of sickness absence, since mental health complaints are associated with prolonged sickness absence spells. ${ }^{17}$ According to a survey conducted in the Netherlands, the average sickness absence duration per sickness absence spell for mental health complaints such as burnout, depression and stress-related complaints constitutes approximately nine weeks. ${ }^{16}$ With respect to muskulo-skeletal and mental 
health complaints, longer duration of sickness absence is associated with a reduced probability of returning to work. The longer the duration of sickness absence, the less likely it becomes that the employee will return to work. ${ }^{18-21}$ Common mental disorders, such as depression and anxiety were found to be significant predictors of longer time until occupational rehabilitation. ${ }^{22}$ Possible explanations for the difficulty in returning to work are the illness severity in this stage, and the fact that the health complaints may be intertwined with the work situation, since work characteristics may have caused the mental health problems. ${ }^{23}$ Furthermore, sickness absence is known to have a multi-factorial etiology, which may include physical and/or mental health complaints, personal factors, lifestyle factors, work-related factors and factors related to private situation. ${ }^{24,25}$ The strength and combination of these factors differs between employees and determines if employees go on sick leave or not and may also complicate return to work. Most interventions for employees with mental health problems still have a rather curative character, which are aimed at rehabilitation of employees already on sick leave. ${ }^{26-28}$ The effects of treatment and rehabilitation of employees on sick leave, in particular due to mental health complaints, are still limited and even a reduction of health complaints does not necessarily lead to work resumption. ${ }^{28-30}$

Therefore, a preventive approach was expected to be more effective. A prerequisite for such a preventive strategy is the ability to identify employees at high risk of long-term sickness absence. In previous research, Kant et al. developed a screening questionnaire to predict future sickness absence among employees. Kant et al. demonstrated that it is possible to identify employees at high risk of future sickness absence by means of a screening questionnaire, called the Balansmeter in Dutch. ${ }^{31}$ Furthermore, in an additional study, it was demonstrated that the timing of preventive intervention among these employees, that is before sickness absence occurs, proved successful in the prevention of long-term sickness absence. ${ }^{32}$ Nevertheless, these studies were conducted among a general working population, in which the risk of future sickness absence could be due to many different causes, and the interventions applied in the studies included a wide range of treatments commonly used in occupational health care.

\section{Focus on mental health and depressive complaints}

The aforementioned studies by Kant et al. proved that early intervention among high-risk employees was effective in the prevention of long-term sickness absence. A study by Taimela et al. also found that an occupational health care intervention was effective in the reduction of total number of sickness absence days in employees identified at high or intermediate risk of sickness absence based on the presence of self-reported physical or psychological complaints. ${ }^{33}$ These findings raised the question of how this preventive strategy could be further improved. A preventive strategy aimed at a more specific target population, including a preventive intervention specifically targeted to this target population, was assumed to be even more effective in the prevention of long-term sickness 
absence. The choice for a suitable target population was based on the two main causes of longterm sickness absence, as mentioned before these include muskulo-skeletal complaints and mental health complaints. Since mental health complaints now are the leading cause of sickness absence in the Netherlands and among most high-income countries, it was decided to focus on mental health complaints, and in particular on depressive complaints. Recent studies have shown that depressive complaints constitute a substantial part of the mental health complaints in the working population. ${ }^{19,}$ ${ }^{34,35}$ In a study conducted by Andrea et al, in which the Hospital Anxiety and Depression Scale was used to assess depressive complaints, a prevalence of mild depressive complaints of $15.8 \%$ was found in women and of $18.7 \%$ in men. ${ }^{36}$ Depressive complaints are not only highly prevalent among employees they also have many consequences. When depressive complaints become more severe and persistent they can lead to (major) depression, which can be a condition with extreme suffering and severe disabilities. According to the World Health Organisation (WHO), depression will be the leading cause of work disability by $2020 .{ }^{37}$ People with depression are known to make more frequent use of health services. ${ }^{6,38,39}$ Besides an increase in health care use, depressive complaints are known to be associated with sickness absence. ${ }^{22,35,40-42}$ Even mild levels of depressive complaints have been found to be associated with future (long-term) sickness absence. ${ }^{6,43}$ Furthermore, more severe depressive complaints have been found to be associated with a higher risk of long-term sickness absence and longer sickness absence duration. ${ }^{40,44}$ Pronounced gender differences are reported in the literature concerning sickness absence due to depression. In most studies, the risk of sickness absence due to depression appeared higher among women than among men. Regarding duration of sickness absence, longer sickness absence spells are often higher among women, but not all studies are consistent on this. ${ }^{45}$ Besides sickness absence, depressive complaints may cause reduced job performance, such as loss of productivity, due to for example loss of concentration and initiative. ${ }^{41,46}$ In the Netherlands, the annual costs of depressive disorders are estimated at 5000 Euros per capita, most of which are due to production losses. ${ }^{47}$

In 2005, the first evidence based guideline for diagnostics and treatment of adults with depression was published. ${ }^{48}$ This guideline describes instruments for the screening, classification and determination of severity of depression, different kinds of treatments and interventions. However, prevention of depression was not mentioned in this guideline. Treatment of depression by occupational physicians is established in the guideline published by the Netherlands Society of Occupational Medicine (NVAB) in 2000 and updated in 2007. ${ }^{49}, 50$ This guideline aims at prevention of long-term sickness absence in already sick-listed employees. The Partnership Depression Prevention (PDP), initiated by the Ministry of Health, Welfare and Sports, and in which the NVAB participates, aims to consolidate prevention of depression nationally and locally. The document on prevention of depression, published by the PDP in 2010, describes the need for prevention of depression by occupational physicians, and it provides concrete interventions and tools for prevention of depression. ${ }^{51}$ However, although the Dutch Health Care Insurance Board included depression prevention in the basic health care package of the health insurance, occupational physicians are not able to receive compensation by the health insurance company yet, since occupational physicians are not mentioned as deliverers of primary health care. 
Despite the presence of a guideline for prevention of depression among employees, the practical organization is still not optimal.

Interventions and treatments for depressive complaints among workers mostly consist of curative interventions mainly consisting of antidepressant medication or psychological treatment, aiming at reduction of depressive symptoms. ${ }^{52,53}$ Studies conducted by Cuijpers et al., in which the effect of a preventive intervention for depression was examined, showed that the risk of depression could be decreased with $20-30 \% .{ }^{54,55}$ However, as mentioned before, the effects of treatment and rehabilitation of employees on sick leave due to mental health complaints are still limited. ${ }^{28,30}$

\section{Preventive strategy}

Based on the limited effects of treatment and rehabilitation of employees on sick leave due to mental health complaints, the high prevalence and high burden of disease of depressive complaints and (major) depression, and the strong relationship between depressive complaints and (future) longterm sickness absence, it was hypothesized that a preventive strategy may be more effective. Since major depression has a strong association with sickness absence, employees with major depression may temporarily have left the labor force or may not be at work at all anymore. Besides depressive complaints causing sickness absence, the opposite relationship has also been established. Sickness absence can cause and predict mental health complaints, such as depressive complaints and (major) depression. ${ }^{4,56}$ For these reasons, the preventive intervention should aim at prevention of sickness absence, but also on prevention of major depression. Therefore, the focus is on employees with a mild level of depressive complaints who are still at work.

The main hypothesis of this thesis is that a preventive strategy aimed at a more specific target population, consisting of employees who are at high risk of future sickness absence and who have a mild level of depressive complaints, will be effective in the prevention of both long-term sickness absence and major depression. The focus on employees at high risk of future sickness absence and with mild depressive complaints, who are still work, requires the development of a new specific intervention targeted to this population.

Prevention can be distinguished in primary, secondary and tertiary prevention. Primary prevention aims to avoid the development of a disease/condition. Most population-based health promotion activities are primary preventive measures. Secondary prevention aims at early recognition and early intervention in people who have some preliminary symptoms of the condition to prevent progression of the disease and emergence of symptoms, while tertiary prevention aims to reduce the negative impact of an already established disease by restoring function and reducing diseaserelated complications.

Both primary and secondary prevention can also be described by the classification introduced by Gordon in $1987 .{ }^{57}$ According to this classification primary prevention is defined as universal 
prevention, which addresses an entire population, regardless of their risk status to prevent the onset of a disease/condition. All individuals are provided with information and skills necessary to prevent the problem. In the classification system of Gordon, secondary prevention is divided into selective prevention and indicated prevention. Selective prevention focuses on groups with an increased risk of developing the disease/condition. Indicated prevention is directed at people who have some symptoms already, but who do not meet the criteria for the full-blown disease/condition. This group may be in an early stage of the disease/condition. In our study, identification of employees with a mild level of depressive complaints belongs to indicated prevention.

Prevention of long-term sickness absence can be considered primary prevention, because the aim is to avoid employees going on sick leave. However, since we aim to identify employees with 'an early stage of the condition', which in our study means identification of those employees at high risk of sickness absence, the type of intervention can better be defined as secondary prevention. In particular, this type of prevention concerns selective prevention, since we also aim to offer a specific treatment to this specific population of high-risk employees. Prevention of major depression in our study can be considered as secondary prevention, and in particular as indicated prevention, since the focus is on identification and treatment of employees with mild depressive symptoms in order to prevent major depression.

\section{General aim}

The general aim of this thesis is to examine if a preventive strategy aimed at employees who are at high risk of future sickness absence and who have a mild level of depressive complaints is effective in the prevention of long-term sickness absence and major depression.

\section{Research questions}

The general aim includes two main research questions.

1 Is it possible to identify employees at high risk of future sickness absence and with mild to severe depressive complaints?

2 Is a preventive intervention, specifically developed for employees who are at high risk of future sickness absence and who have mild to severe depressive complaints, effective in the prevention of long-term sickness absence and major depression?

In the remainder of this chapter, background information on the general aim and research questions will be given, and subsequently the chapters will be introduced in which the research questions will be addressed. 


\section{Identification of the target population through screening}

To select employees for participation in a preventive intervention, several selection methods can be considered. Since the aim was to select employees with relatively mild (health) complaints and an increased risk for a future event, personal enlistment of employees or recognition of these mild level of complaints by supervisors or counselors from the occupational health services, was not very likely to occur. Moreover, the use of diagnostic interviews to detect employees with depressive complaints was not suitable in such a large-scale company. A more feasible and more efficient selection method consists of a large-scale method of screening. In previous research by Kant et al. a screening questionnaire, called Balansmeter, was developed to identify employees at high risk of future sickness absence. The Balansmeter proved to be effective in predicting future sickness absence.

From an ethical perspective, several criteria must be met before screening is allowed. Based on criteria from Wilson and Jungner, ${ }^{58}$ screening for a (pre-phase, marker of a) disease is amongst others allowed when the condition screened for concerns a major health problem, when an effective screening instrument is available as well as an effective treatment.

To ensure that the intervention will be directed at those employees who benefit the most, to restrict the number of false-positively classified healthy workers and to spend the available money in the most optimal way, a screening instrument with high specificity was preferred.

For the present study, a screening instrument was used for the selection of employees. Besides the Balansmeter, another screening questionnaire was included to identify employees with mild to severe depressive complaints. The Hospital Depression and Anxiety Scale (HAD-D) has been found to be a valid questionnaire to assess the presence and severity of depressive complaints among employees and was therefore included in the screening instrument. ${ }^{36}$ The selection procedure of employees by means of a screening questionnaire is an objective and relatively easy way to address many employees at the same time, in which the privacy of employees can be guaranteed. However, selection of employees with a screening questionnaire incorporates several problems, such as nonresponse, employees who are not willing or motivated to participate in a (non-requested) preventive intervention.

Identification of those employees at high risk of future sickness absence and with mild to severe depressive complaints by means of an objective screening questionnaire involves the problem, characteristic for research on prevention, of the willingness to be screened and the need for motivation to participate in a preventive intervention. ${ }^{32,59}$ The preventive strategy is directed at employees with relatively mild (health) complaints and who are not on sick leave yet. The preventive intervention can be considered as a rather healthy intervention. This is in line with developments in occupational health care, in which the focus of treatment of sick employees has shifted from reintegration to improvement of personal wellbeing and health of relatively healthy and still working employees. When using an objective selection process to identify employees with relatively mild 
(health) complaints and an increased risk for a future event, it may be possible that employees do not experience complaints or their status of being at high risk, at the moment of completing the screening questionnaire. However, the advantage of an objective screening method is that those employees who are likely to benefit most from the intervention will be selected, in stead of treatment based on subjective selection of employees who ask help for complaints themselves, but who may not be at risk for sickness absence. A previous study by Kant et al. ${ }^{32}$ studied if the timing of early intervention was effective, so early intervention before sickness absence occurred. In that study, employees were selected by the Balansmeter at high risk of future sickness absence out of a general working population. The selected employees all received a consultation with an occupational physician. The consultation aimed to identify what kind of problems caused the increased risk of future sickness absence and to guide employees to the appropriate kind of treatment.

In the present study, a more specific population of employees was selected by the screening instrument, and a specific intervention was developed for this population. To improve actual participation in the preventive intervention, employees were contacted by a company counselor and invited for a consultation at their own work place. Employees of the banking company under study are rather familiar with their occupational health services, since they have contacts with their employees on a regular basis, and the occupational health services are easy accessible for the employees.

\section{Preventive intervention}

Besides the availability of an objective screening instrument to identify employees at high risk of future sickness absence and with depressive complaints, and sufficient motivation to participate in the preventive intervention, an effective preventive intervention must be available to prevent longterm sickness absence and major depression. In the previous study by Kant et al. ${ }^{32}$ which examined the effect of timing of early intervention on the prevention of sickness absence, a wide range of common interventions used in occupational health care was applied since selected employees were at high risk of sickness absence due to any cause. The target population of the present study includes employees at high risk of sickness absence and with mild to severe depressive complaints. In this study, the preventive intervention is directed at employees who are still at work and who have relatively mild health complaints. As described earlier, depressive complaints are known to be associated with sickness absence themselves. Besides depressive complaints causing sickness absence, the opposite relationship has also been established. Sickness absence can cause and predict mental health complaints, such as depressive complaints and (major) depression. ${ }^{4,56}$ Since mental health and sickness absence have been demonstrated to be intertwined and to influence each other, the preventive intervention should aim at prevention of sickness absence, but also on prevention of major depression.

Numerous studies have investigated the effectiveness of different treatments for depression. ${ }^{60,61}$ Strong evidence exists that cognitive behavioral therapy (CBT) provided by trained experts is an 
effective treatment for depression, ${ }^{62-65}$ as well as computerized-CBT. ${ }^{66,67}$ CBT has also been proven effective in reducing sickness absence. Computerized-CBT in employees with depression or anxiety proved also effective in reducing the number of sickness absence days over 8-months of follow-up. ${ }^{68}$ Another treatment often used and which has been proved effective in the treatment of depression is problem solving therapy/treatment (PST). ${ }^{69-74}$ The rationale underpinning Problem Solving Therapy is that patients' psychological symptoms are caused by everyday problems they are experiencing. ${ }^{69}$,

${ }^{72}$ Hence, if patients' problems can be resolved, their symptoms will improve. ${ }^{72}$ Problem solving therapy has been proven effective with respect to the experience of disabilities and reduction of sickness absence in employees with non-specific low back pain. ${ }^{75}$ Regarding these results and the rationale, PST was assumed to be beneficial in the prevention of future sickness absence as well as (major) depression. PST was expected to act upon the determinants causing the high risk of future sickness absence, such as health complaints, work-related factors, and private situation-related factors. And because mild depressive complaints may be a reaction to stresses and strains of life, coping interventions, such as problem solving therapy, would seem an ideal treatment. ${ }^{76}$ Problem solving treatment including cognitive behavioral elements was expected to be most effective in the prevention of both long-term sickness absence and depressive complaints. Besides the two main outcomes, depressive complaints and sickness absence, the intervention was expected to have a positive effect on more general outcomes as well. General well-being, (mental) health perception and perception of work characteristics, such as psychological job demands and decision latitude are associated with depressive complaints and/or sickness absence and may improve due to the intervention as well. In conclusion, CBT and PST are effective in the treatment of depression and some evidence showed positive effects on the reduction of sickness absence.

A preventive intervention aimed at prevention of both sickness absence and major depression among employees at high risk of sickness absence and with mild to severe depressive complaints, but who are still at work, was not yet available. Therefore, a new protocol based on PST and CBT was specifically developed for our target population. The focus of the intervention was mainly on workrelated (health) complaints, and the intensity of the intervention was adapted to the mild level of complaints. The hypothesis was that the new intervention would first reduce the level of depressive complaints, and consequently would prevent long-term sickness absence.

\section{Study design and setting}

Both research themes are part of a randomized controlled trial (RCT), in which employees of a large banking company, the ABN AMRO Banking Company, in the Netherlands participated. The study was conducted in corporation with the Occupational Health Services Beter. The occupational health services have many companies as their clients, the banking company the present study was conducted at is one of these clients. The study was conducted in 2007 and 2008. In January 2007, 25,404 people were employed at the banking company, and in January 2008 the banking company 
included 24,313 employees. The total sickness absence rate was $3.83 \%$ in 2007 and $3.77 \%$ in 2008 . These rates are already lower than the Dutch sickness absence rates, however, reduction of sickness absence remains one of their major spearheads.

In the RCT, the preventive intervention was compared with the care as usual provided by the occupational health services. According to Dutch legislation, employees on sick leave receive socio-medical counseling and treatment according to the nationwide guidelines. Occupational health care originally focused on improvement of working conditions, such as elimination and reduction of chemical, ergonomical and physical exposures, indicating a preventive approach. Since the introduction of the Sickness Absence Reduction Act in 1994, the focus shifted to reduction of sickness absence mainly through rehabilitation of employees who were on sick leave already. In the last decade, the focus changes back to prevention and health management/promotion in which sustainable employability is the central theme. New trends in public health have developed, such as ageing of the population, the increase in prevalence of chronic conditions, such as obesity, metabolic syndrome and mental health complaints. However, occupational health care is still largely based on a reactive approach. This means that intervention is initiated when the employee or supervisor recognize (health) difficulties. Preventive measures are also still, to a large extent, aimed at the total working population instead of specific high risk populations. The focus on a preventive strategy aimed at a population of employees at high risk of a disease or future event may be a new opportunity for occupational health services. However, such a preventive strategy requires adaptations in the organization of the occupational health care. In the present study, the preventive intervention was conducted by psychologists from a company consisting of a nationwide network of registered psychologists, called Cenzo B.V. This company regularly provides psychological health care for the occupational health services Beter.

\section{Outline of the thesis}

First the basic assumption of this thesis was studied, that is, the relationship between different levels of depressive complaints and sickness absence. In chapter 2 , the results of the cross-sectional and longitudinal relationship between depressive complaints and sickness absence, in particular sickness absence duration and time to onset of the first sickness absence spell are described. This chapter was based on data from the Maastricht Cohort Study, a prospective cohort study which was set up in 1998 as part of the Netherlands concerted research action on Fatigue at Work, including over 12,000 employees from various sectors and trades at baseline measurement. ${ }^{77}$ The remaining chapters are based on results from the RCT. In chapter 3 , the predictive properties of the screening questionnaire in predicting future long-term sickness absence in employees identified both at high risk of sickness absence and with mild to severe depressive complaints were examined. Chapter 4 presents the results of a study examining the relationship between the experience of health complaints and help seeking behavior in employees screened for the risk of future sickness absence and/or screened for mild to 
severe depressive complaints. Chapter 5 presents the results of the randomized controlled trial on the efficacy of the preventive intervention, specifically developed for employees indentified both at high risk of sickness absence and with mild to severe depressive complaints, on the prevention of long-term sickness absence and major depression. Chapter 6 further describes the effect of protocol adherence on long-term sickness absence and depressive complaints. The influence of variations between individual interventions, such as the number of sessions, on the outcomes was examined. Chapter 7, the general discussion, presents a more general overview of the results and conclusions of the studies described in the present thesis, their strengths and weaknesses, practical implications and implications for future research. 


\section{References}

1. Marmot M, Feeney A, Shipley M, North F, Syme SL. Sickness absence as a measure of health status and functioning: from the UK Whitehall II study. J Epidemiol Community Health 1995;49:124-30.

2. Kivimaki M, Forma P, Wikstrom J, Halmeenmaki T, Pentti J, Elovainio M, et al. Sickness absence as a risk marker of future disability pension: the 10-town study. J Epidemiol Community Health 2004;58:710-1.

3. Kivimaki M, Head J, Ferrie JE, Shipley MJ, Vahtera J, Marmot MG. Sickness absence as a global measure of health: evidence from mortality in the Whitehall II prospective cohort study. BMJ 2003;327(7411):364.

4. Kivimaki M, Head J, Ferrie JE, Singh-Manoux A, Westerlund H, Vahtera J, et al. Sickness absence as a prognostic marker for common chronic conditions: analysis of mortality in the GAZEL study. Occup Environ Med 2008;65:820-6.

5. Borritz M, Rugulies R, Christensen KB, Villadsen E, Kristensen TS. Burnout as a predictor of self-reported sickness absence among human service workers: prospective findings from three year follow-up of the PUMA study. Occup Environ Med 2006;63:98-106.

6. Birnbaum HG, Kessler RC, Kelley D, Ben-Hamadi R, Joish VN, Greenberg PE. Employer burden of mild, moderate, and severe major depressive disorder: mental health services utilization and costs, and work performance. Depress Anxiety;27:78-89.

7. Grundemann RWM, van Vuuren CV. Securing health together. London: HSE, 2000.

8. Statistics Netherlands. Statistisch jaarboek 2005 (Statistical Yearbook 2005). Voorburg/Heerlen: Centraal Bureau voor de Statistiek; 2005.

9. Jehoel-Gijsbers G. Rapportage ziekteverzuim, arbeidsongeschiktheid en arbeidsparticipatie. Den Haag: Sociaal en Cultureel Planbureau; 2010.

10. Statistics Netherlands. National statistics on sick leave, frequency, period of absence. Heerlen/Voorburg, the Netherlands. Available at http://www.statline.cbs.nl. Accessed at 2 September 2010.

11. Hartman H, Kartopawiro J, Jansen F. De Nederlandse economie. [The Dutch Economy; statistics on sick leave and work disability]. Heerlen/Voorburg: Statistics Netherlands; 2009.

12. Gjesdal S, Ringdal PR, Haug K, Maeland JG. Long-term sickness absence and disability pension with psychiatric diagnoses: a population-based cohort study. Nord J Psychiatry 2008;62:294-301.

13. Lund T, Kivimaki M, Labriola M, Villadsen E, Christensen KB. Using administrative sickness absence data as a marker of future disability pension: the prospective DREAM study of Danish private sector employees. Occup Environ Med. 2008;65:28-31.

14. Confederation of British Industry. Attending to Absence CBI/AXA Absence and Labour Turnover Survey 2006. CBI; 2007.

15. Lund T, Labriola M, Villadsen E. Who is at risk for long-term sickness absence? A prospective cohort study of Danish employees. Work 2007;28:225-30.

16. Nederlands Centrum voor Beroepsziekten. Beroepsziekten in cijfers 2009; 2009.

17. Shiels C, Gabbay MB, Ford FM. Patient factors associated with duration of certified sickness absence and transition to long-term incapacity. Br J Gen Pract 2004;54:86-91.

18. Frank JW, Kerr MS, Brooker AS, DeMaio SE, Maetzel A, Shannon HS, et al. Disability resulting from occupational 
low back pain. Part I: What do we know about primary prevention? A review of the scientific evidence on prevention before disability begins. Spine 1996;21:2908-17.

19. Henderson M, Glozier N, Holland Elliott K. Long-term sickness absence. BMJ 2005;330:802-3.

20. Watson PJ, Main CJ, Waddell G, Gales TF, Purcell-Jones G. Medically certified work loss, recurrence and costs of wage compensation for back pain: a follow-up study of the working population of Jersey. Br J Rheumatol 1998;37:82-6.

21. Williams DA, Feuerstein M, Durbin D, Pezzullo J. Health care and indemnity costs across the natural history of disability in occupational low back pain. Spine 1998;23:2329-36.

22. Nieuwenhuijsen K, Verbeek JH, de Boer AG, Blonk RW, van Dijk FJ. Predicting the duration of sickness absence for patients with common mental disorders in occupational health care. Scand J Work Environ Health 2006;32:67-74.

23. Janssen N, van den Heuvel WP, Beurskens AJ, Nijhuis FJ, Schroer CA, van Eijk JT. The Demand-Control-Support model as a predictor of return to work. Int J Rehabil Res 2003;26:1-9.

24. Bultmann U, Huibers MJ, van Amelsvoort LP, Kant I, Kasl SV, Swaen GM. Psychological distress, fatigue and long-term sickness absence: prospective results from the Maastricht Cohort Study. J Occup Environ Med 2005;47:941-7.

25. Hensing G, Alexanderson K. The association between sex segregation, working conditions, and sickness absence among employed women. Occup Environ Med 2004;61:e7.

26. Hlobil H, Staal JB, Twisk J, Koke A, Ariens G, Smid T, et al. The effects of a graded activity intervention for low back pain in occupational health on sick leave, functional status and pain: 12-month results of a randomized controlled trial. J Occup Rehabil 2005;15:569-80.

27. Rost $\mathrm{K}$, Smith JL, Dickinson M. The effect of improving primary care depression management on employee absenteeism and productivity. A randomized trial. Med Care 2004;42:1202-10.

28. van Oostrom SH, van Mechelen W, Terluin B, de Vet HC, Knol DL, Anema JR. A workplace intervention for sicklisted employees with distress: results of a randomised controlled trial. Occup Environ Med 2010;67:596602.

29. Brouwers EP, Tiemens BG, Terluin B, Verhaak PF. Effectiveness of an intervention to reduce sickness absence in patients with emotional distress or minor mental disorders: a randomized controlled effectiveness trial. Gen Hosp Psychiatry 2006;28:223-9.

30. Huibers MJ, Beurskens AJ, Van Schayck CP, Bazelmans E, Metsemakers JF, Knottnerus JA, et al. Efficacy of cognitive-behavioural therapy by general practitioners for unexplained fatigue among employees: Randomised controlled trial. Br J Psychiatry 2004;184:240-6.

31. Kant IJ, Jansen NW, van Amelsvoort LG, Swaen GM, van Leusden R, Berkouwer A. Screening questionnaire Balansmeter proved successful in predicting future long-term sickness absence in office workers. J Clin Epidemiol 2009;62:408-14.

32. Kant I, Jansen NW, van Amelsvoort LG, van Leusden R, Berkouwer A. Structured early consultation with the occupational physician reduces sickness absence among office workers at high risk for long-term sickness absence: a randomized controlled trial. J Occup Rehabil 2008;18:79-86.

33. Taimela S, Malmivaara A, Justen S, Laara E, Sintonen $\mathrm{H}$, Tiekso J, et al. The effectiveness of two occupational 
health intervention programmes in reducing sickness absence among employees at risk. Two randomised controlled trials. Occup Environ Med 2008;65:236-41.

34. Koopmans PC, Roelen CA, Groothoff JW. Sickness absence due to depressive symptoms. Int Arch Occup Environ Health 2008;81:711-9.

35. Laitinen-Krispijn S, Bijl RV. Mental disorders and employee sickness absence: the NEMESIS study. Netherlands Mental Health Survey and Incidence Study. Soc Psychiatry Psychiatr Epidemiol 2000;35:71-7.

36. Andrea H, Bultmann U, Beurskens AJ, Swaen GM, van Schayck CP, Kant IJ. Anxiety and depression in the working population using the HAD Scale--psychometrics, prevalence and relationships with psychosocial work characteristics. Soc Psychiatry Psychiatr Epidemiol 2004;39:637-46.

37. World Health Organization. World Health Report 2000. Geneva, Switzerland: World Health Organization; 2000.

38. Cuijpers $\mathrm{P}$, de Graaf R, van Dorsselaer $\mathrm{S}$. Minor depression: risk profiles, functional disability, health care use and risk of developing major depression. J Affect Disord 2004;79:71-9.

39. Lerner D, Henke RM. What does research tell us about depression, job performance, and work productivity? J Occup Environ Med 2008;50:401-10.

40. Bultmann $U$, Rugulies $R$, Lund $T$, Christensen KB, Labriola $M$, Burr $H$. Depressive symptoms and the risk of long-term sickness absence: a prospective study among 4747 employees in Denmark. Soc Psychiatry Psychiatr Epidemiol 2006;41:875-80.

41. Lerner D, Adler DA, Chang H, Lapitsky L, Hood MY, Perissinotto C, et al. Unemployment, job retention, and productivity loss among employees with depression. Psychiatr Serv 2004;55:1371-8.

42. Stansfeld S, Feeney A, Head J, Canner R, North F, Marmot M. Sickness absence for psychiatric illness: the Whitehall II Study. Soc Sci Med 1995;40:189-97.

43. Hjarsbech PU, Andersen RV, Christensen KB, Aust B, Borg V, Rugulies R. Clinical and non-clinical depressive symptoms and risk of long-term sickness absence among female employees in the Danish eldercare sector. J Affect Disord, Published Online First: 24 August 2010. doi:10.1016/j.jad.2010.07.033

44. Dewa CS, Goering P, Lin E, Paterson M. Depression-related short-term disability in an employed population. J Occup Environ Med 2002;44:628-33.

45. Hensing G, Alexanderson K, Allebeck P, Bjurulf P. Sick-leave due to psychiatric disorder: higher incidence among women and longer duration for men. Br J Psychiatry 1996;169:740-6.

46. Kessler RC, Akiskal HS, Ames M, Birnbaum H, Greenberg P, Hirschfeld RM, et al. Prevalence and effects of mood disorders on work performance in a nationally representative sample of U.S. workers. Am J Psychiatry 2006;163:1561-8.

47. Smit F, Cuijpers P, Oostenbrink J, Batelaan N, de Graaf R, Beekman A. Costs of nine common mental disorders: implications for curative and preventive psychiatry. J Ment Health Policy Econ 2006;9:193-200.

48. Multidisciplinaire richtlijn Depressie. Richtlijn voor de diagnostiek en behandeling van volwassen cliënten met een depressie. Utrecht: Trimbos Instituut in opdracht van Landelijke Stuurgroep Multidisciplinaire Richtlijnontwikkeling in de GGZ; 2005.

49. van der Klink JJ. ed. Guidelines for mental health problems. Eindhoven: NVAB (Dutch Association of Occupational Physicians); 2000. 
50. van der Klink, JJ. ed. Richtlijn: handelen van de bedrijfsarts bij werkenden met psychische problemen. Utrecht: Nederlandse Vereniging voor Arbeids- en Bedrijfsgeneeskunde (NVAB) (Dutch Association of Occupational Physicians); 2007.

51. Loo M, Nauta N. Depressiepreventie. Handreiking voor bedrijfsartsen versie 1.0. Utrecht: Partnership Depressiepreventie; 2010.

52. Birnbaum HG, Ben-Hamadi R, Kelley D, Hsieh M, Seal B, Kantor E, et al. Assessing the relationship between compliance with antidepressant therapy and employer costs among employees in the United States. J Occup Environ Med 2010;52:115-24.

53. Martin A, Sanderson K, Cocker F. Meta-analysis of the effects of health promotion intervention in the workplace on depression and anxiety symptoms. Scand J Work Environ Health 2009;35:7-18.

54. Cuijpers P, Van Straten A, Smit F. Preventing the incidence of new cases of mental disorders: a meta-analytic review. J Nerv Ment Dis 2005;193:119-25.

55. Cuijpers P, van Straten A, Smit F, Mihalopoulos C, Beekman A. Preventing the onset of depressive disorders: a meta-analytic review of psychological interventions. Am J Psychiatry 2008;165:1272-80.

56. Melchior M, Ferrie JE, Alexanderson K, Goldberg M, Kivimaki M, Singh-Manoux A, et al. Using sickness absence records to predict future depression in a working population: prospective findings from the GAZEL cohort. Am J Public Health 2009;99:1417-22.

57. Gordon R. Een operationele classificatie van ziektepreventie. In Steinberg, J. A. en Silverman, M. M. (eds.), Verhinderend Geestelijke Wanorde, Rockville, MD: De V.S. Ministerie van Gezondheid en de Menselijke Diensten, 1987.

58. Wilson JMG. Principles and Practice of Screening for Disease. WHO Chron 1968;22:473.

59. Duijts SF, Kant I, Swaen GM. Advantages and disadvantages of an objective selection process for early intervention in employees at risk for sickness absence. BMC Public Health 2007;7:67.

60. Gloaguen V, Cottraux J, Cucherat M, Blackburn IM. A meta-analysis of the effects of cognitive therapy in depressed patients. J Affect Disord 1998;49:59-72.

61. Wampold BE, Minami T, Baskin TW, Callen Tierney S. A meta-(re)analysis of the effects of cognitive therapy versus 'other therapies' for depression. J Affect Disord 2002;68:159-65.

62. Beck AT. The current state of cognitive therapy: a 40-year retrospective. Arch Gen Psychiatry 2005;62:9539.

63. Butler AC, Chapman JE, Forman EM, Beck AT. The empirical status of cognitive-behavioral therapy: a review of meta-analyses. Clin Psychol Rev 2006;26:17-31.

64. DeRubeis RJ, Hollon SD, Amsterdam JD, Shelton RC, Young PR, Salomon RM, et al. Cognitive therapy vs medications in the treatment of moderate to severe depression. Arch Gen Psychiatry 2005;62:409-16.

65. Hollon SD, Stewart MO, Strunk D. Enduring effects for cognitive behavior therapy in the treatment of depression and anxiety. Annu Rev Psychol 2006;57:285-315.

66. Proudfoot J, Ryden C, Everitt B, Shapiro DA, Goldberg D, Mann A, et al. Clinical efficacy of computerised cognitive-behavioural therapy for anxiety and depression in primary care: randomised controlled trial. $\mathrm{Br} J$ Psychiatry 2004;185:46-54.

67. Wright JH, Wright AS, Albano AM, Basco MR, Goldsmith LJ, Raffield T, et al. Computer-assisted cognitive 
therapy for depression: maintaining efficacy while reducing therapist time. Am J Psychiatry 2005;162:115864.

68. McCrone P, Knapp M, Proudfoot J, Ryden C, Cavanagh K, Shapiro DA, et al. Cost-effectiveness of computerised cognitive-behavioural therapy for anxiety and depression in primary care: randomised controlled trial. $\mathrm{Br} J$ Psychiatry 2004;185:55-62.

69. Bell AC, D'Zurilla TJ. Problem-solving therapy for depression: a meta-analysis. Clin Psychol Rev 2009;29:34853.

70. Cuijpers $P$, van Straten A, Warmerdam L. Problem solving therapies for depression: a meta-analysis. Eur Psychiatry 2007;22:9-15.

71. Dowrick C, Dunn G, Ayuso-Mateos JL, Dalgard OS, Page H, Lehtinen V, et al. Problem solving treatment and group psychoeducation for depression: multicentre randomised controlled trial. Outcomes of Depression International Network (ODIN) Group. BMJ 2000;321:1450-4.

72. Mynors-Wallis L. Does problem-solving treatment work through resolving problems? Psychol Med 2002;32:1315-9.

73. Mynors-Wallis LM, Gath DH, Day A, Baker F. Randomised controlled trial of problem solving treatment, antidepressant medication, and combined treatment for major depression in primary care. BMJ 2000;320:2630.

74. Mynors-Wallis LM, Gath DH, Lloyd-Thomas AR, Tomlinson D. Randomised controlled trial comparing problem solving treatment with amitriptyline and placebo for major depression in primary care. BMJ 1995;310:4415.

75. van den Hout JH, Vlaeyen JW, Heuts PH, Zijlema JH, Wijnen JA. Secondary prevention of work-related disability in nonspecific low back pain: does problem-solving therapy help? A randomized clinical trial. Clin J Pain 2003;19:87-96.

76. Oxman TE, Hegel MT, Hull JG, Dietrich AJ. Problem-solving treatment and coping styles in primary care for minor depression. J Consult Clin Psychol 2008;76:933-43.

77. Mohren D, Jansen N, Amelsvoort L, Kant I. An Epidemiological Approach of Fatigue and Work: Experiences from the Maastricht Cohort Study. Amersfoort: Wilco; 2007. 



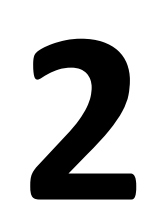

\title{
Depressive complaints as a predictor of sickness absence among the working population
}

\author{
Monique A. S. Lexis $^{1}$ \\ Nicole W.H. Jansen ${ }^{1}$ \\ Ludovic G.P.M. van Amelsvoort ${ }^{1}$ \\ Piet A. van den Brandt ${ }^{1}$ \\ IJmert Kant ${ }^{1}$
}

${ }^{1}$ CAPHRI School for Public Health and Primary Care, Department of Epidemiology, Maastricht University, Maastricht, The Netherlands 


\section{Abstract}

\section{Objective}

To study the relationship between depressive complaints and sickness absence in the working population.

\section{Methods}

Data from a prospective epidemiological cohort $(n=3,339)$ were used. Depressive complaints were measured with the Hospital Anxiety and Depression (HAD-D) Scale. Sickness absence was assessed objectively through individual record linkage with the company registers.

\section{Results}

Higher levels of depressive complaints were associated with a shorter time to first sickness absence spell and a longer duration of sickness absence. In women with mild depressive complaints, the average number of sickness absence days over 10 months follow-up was 27.37 (SD 64.73) days versus 11.01 (SD 30.03) days $(p<0.001)$ in employees scoring within the normal range. In men this was 14.48 (SD 38.73) days versus 7.67 (SD 25.80) days ( $p<0.001)$.

\section{Conclusions}

Prevention of mild depressive complaints might be beneficial in preventing future sickness absence. 


\section{Introduction}

Depression is a common mental disorder affecting about 121 million people worldwide. According to the World Health Organization, depression will be the second leading cause of morbidity by the year $2020 .{ }^{1}$ Annually, $5.8 \%$ of the Dutch population meet the DSM-IV criteria of depressive disorder. ${ }^{2}$ Depending on the definition and measurement of depression reported prevalences of depression and depressive complaints greatly vary in the literature. In the current study, the focus is on a relatively mild level of depressive complaints, which may be viewed as a possible precursor for depressive disorder. ${ }^{3}$ Depressive complaints are characterized by relatively mild to moderate symptoms of depression which have not been classified as a depression according to the commonly used gold standard, the diagnostic interview based on the DSM-IV criteria. In this perspective depressive complaints may be viewed as a continuum of complaints, ranging from no depressive complaints at one end of the spectrum to severe complaints at the other end. The continuum encompasses the total range of complaints that precedes the diagnosis of clinical depression. An annual Dutch report on self-reported depressive complaints showed a prevalence of approximately $10 \%$ percent in the general Dutch population over the period 2002-2005. A prevalence of $8.4 \%$ was found for men and $13.2 \%$ for women. ${ }^{4}$ In the working population depressive complaints are also highly prevalent. Studying the continuum of depressive complaints is particularly relevant in the labor force. Andrea et al. found that depressive complaints, measured by the screening questionnaire Hospital Anxiety and Depression Scale, were even more prevalent in the working population than the general Dutch population. In their study, based on the same cohort as our study, a classification was made based on different levels of depressive complaints. For men, $18.7 \%$ of employees were classified with mild depressive complaints and $7.1 \%$ with moderate depressive complaints. For women, the prevalences were $15.8 \%$ and $6.2 \%$ respectively. ${ }^{5}$

Depressive complaints among employees are a very important topic in the labor force, because they contribute considerably to health care use, loss of productivity and disability days. ${ }^{3,6}$ Since employees with a clinical depression are disabled from work in many cases, a strategy aimed at early identification and treatment of employees with especially mild depressive symptoms might reduce the risk of developing severe depressive complaints and future long-term sickness absence. Consequently, this strategy may also reduce the costs of mental health problems in the workforce. ${ }^{3,}$

7-9 However, the majority of studies on depression among the workforce have focused on major depression as a risk factor for sickness absence. ${ }^{5,10-13}$ Depression is associated with an increased risk of sickness absence for both men and women. ${ }^{8,14,15}$ Pronounced gender differences are also reported in the literature concerning sickness absence due to depression. In most studies, the risk of sickness absence because of depression appeared higher among women than among men, ${ }^{13,16-19}$ but not all studies are consistent on this. Discrepancies between men and women regarding the duration of sickness absence also exist. Most studies show longer sickness absence spells in women than in men. ${ }^{20-22}$ However, other studies show opposite results. ${ }^{14,23}$ 
While many studies have been conducted on the relation between depression and sickness absence, less is known about the impact of different levels of depressive complaints on sickness absence. In a longitudinal study Stansfeld et al. ${ }^{13}$ showed that minor psychiatric disorders, including minor depression, were one of the most common reasons for sickness absence among civil servants. In a prospective study conducted in the Danish general working population, a more than two times higher risk of long-term sickness absence was found in both men and women with severe depressive symptoms, compared with subjects without depressive symptoms. ${ }^{10}$ Many studies showed that a higher level of depressive symptoms was also related to longer sickness absence. ${ }^{12,24-26}$ Workers with major depression were found to have between 1.5 and 3.2 times more short-term disability days over a thirty-day period than other workers. ${ }^{11}$

Information is also scarce on duration and time to onset of sickness absence. This is of particular interest, because duration and time to onset of sickness absence encompass different information. Especially short-term sickness absence is regarded as a coping strategy to reduce health complaints and thus to prevent more serious ill health. ${ }^{27}$ Low or mild levels of health complaints, such as a cold, generally correspond with more frequent but short-term sickness absence. There is a strong relationship between absence frequency and time to onset of a sick leave episode. Short-term sickness absence is predictive of a quick onset of the first sickness absence spell. More severe health complaints, on the other hand, correspond with less frequent but long-term sickness absence. ${ }^{28}$ Individuals with more severe depressive symptoms are more likely to report days of work loss due to depression..$^{29-31}$

The aim of the present paper is to study both cross-sectionally and prospectively the relationships between depressive complaints and sickness absence from work. We hypothesize that relatively mild depressive complaints will result in a higher frequency and a shorter time to onset of the first sickness absence spell and that there will be a dose-response-like relationship between depressive complaints and sickness absence.

Whereas cross-sectional analyses using self-reported sickness absence data give insight into the effects that are already present at baseline measurement, the present study will additionally focus on the prospective relationship between depressive complaints and objective sickness absence using data through company record linkage. We are particularly interested whether different levels of depressive complaints are predictive of increased sickness absence, in terms of time to onset of sickness absence and sickness absence duration.

When conducting a study on levels of depressive complaints in relation to sickness absence, one should carefully consider the multi-factorial etiology of sickness absence. ${ }^{27}$ Hence, one should take into account differences in demographic characteristics, work related factors and health status. 


\section{Methods}

\section{Maastricht Cohort Study}

Data were used from an ongoing large-scale prospective epidemiological cohort study in the Netherlands, addressing a broad range of work related and non-work related factors, individual factors and (mental) health status. At baseline in May 1998, this Maastricht Cohort Study on Fatigue at Work surveyed a population of 12,140 employees from 45 different companies and organizations, representing a baseline response rate of $45 \%$. Employees were followed by means of ten consecutive self-administered questionnaires. Baseline characteristics of the study population and a non-response analysis have been described elsewhere. ${ }^{32}$

\section{Study population}

The scale used to measure depressive complaints was first included in the ninth questionnaire (T8) of the study, in January 2001. Therefore, T8 will constitute the baseline measurement for the current study. At T8, 8,033 employees received the questionnaire and, of these, 7,482 employees completed and returned the questionnaire (response rate 93.1\%). First, 77 records with incomplete and/or incorrect data on self-reported sickness absence variables were removed from the database. Second, female employees who were pregnant at the time of completing the questionnaire $(n=28)$ were excluded to avoid counting spells specifically related to pregnancy leave, resulting in a study population of 7,377 employees, 1,992 women and 5,385 men for the cross-sectional analyses.

Longitudinal data derived from register-based information on sickness absence from the participating companies and organizations in the Maastricht Cohort Study were collected between 1998 and 2001. For the original Maastricht Cohort Study, 45 companies agreed to provide us with sickness absence data until December 2000. Thirteen out of the 45 companies were able to provide us with sickness absence data for one additional year, the year 2001, which were needed for the current study. Objective sick leave data were available for 3,580 participants at T8. In this case, selective drop-out of participants is rather unlikely to occur since objective sickness absence data concern data on company level instead of an individual level. For the prospective analyses, the same exclusion criteria were applied as for the cross-sectional analyses and we additionally excluded those employees who reported themselves fully or partially absent from work at T8 to study incident sickness absence exclusively. Women were also excluded if they were on sick leave because of pregnancy or maternity leave during follow-up. After application of the exclusion criteria the study population for the prospective analyses included 3,339 employees, of which 2,666 were men and 673 were women. 


\section{Measurements}

\section{Depressive complaints}

The Hospital Anxiety and Depression (HAD) scale was used to measure the presence and severity of depressive complaints. The HAD is a 14 item self-report questionnaire that was originally developed to indicate the presence and severity of both anxiety (HAD-A) and depression (HAD-D) separately. ${ }^{33}$ Both the HAD-A and the HAD-D consist of 7 items which are scored on a four point Likert scale (0 to 3 ), resulting in a range of 0 to 21 . In this study, only the HAD-D was used. A score on the Likert scale represents a combination of the number and severity of depressive complaints. A higher score on the HAD-D is indicative for having more severe depressive complaints. Three categories of depressive complaints were originally defined by Zigmond and Snaith in 1983. A score of less than 8 points was defined as non case of depression; a score of 8 points and more was defined as a possible case of depression and 11 points or more as a subclinical case of depression. ${ }^{33,34}$ Although the HAD scale was originally developed to identify (possible) caseness of anxiety disorders and depression among patients in non-psychiatric hospital clinics, the questionnaire was recently found to perform well in assessing the symptom severity and caseness of anxiety disorders and depression in somatic, psychiatric and primary care patients and in the general population. Cronbach's alphas for HAD-D were .86 and .85 for females and males respectively. ${ }^{5}$ In subsequent research on the HAD scale, Snaith did not present the categories as a probability of the presence of depression but presented a different definition of the categories. These categories, which are mutually exclusive, were presented as a division of depressive complaints into three ranges: 1 ) normal (less than 8 points), 2) mild (8-10 points), and 3) moderate and severe (11 points or more). ${ }^{35}$ The latter description is in line with our approach of depressive complaints, as defined earlier, as a continuum.

\section{Sickness absence}

Data about sickness absence were gathered from objective organizational absence records and also from the self-report questionnaire. In the present study, data on self-reported sickness absence were only used to perform the cross-sectional analyses and for exclusion of prevalent cases for the prospective analyses. As outcome measure for the longitudinal analyses, we used objective companyregistered sickness absence data, which were obtained by record linkages with the company sickness absence registry systems. For the present study sickness absence data from the year 2001 were used. After sending out the questionnaires in January 2001, a follow-up period of ten months (March-December 2001) was maintained. A time lag of two months was used between the time of employees receiving the questionnaire and the measurement of sickness absence, as the time of the returning of the questionnaires and the processing of the data at most could take two months. All information regarding time to onset of first sickness absence spell and total number of days absent from work over the ten months of follow-up was measured through record linkage on an individual level with the company registers on sickness absence. 


\section{Potential confounding factors}

Several potential confounding factors should be controlled for when studying the relationship between depressive complaints and sickness absence. Because of earlier reported gender differences both with regard to depressive complaints and sickness absence, all analyses were conducted for men and women separately. In this study several domains of potential confounding factors were taken into account, that is demographic factors, health, work related factors and lifestyle factors. Educational level (low, medium, high), smoking (yes/no), living alone (yes/no), the presence of a long-term illness (psychological illnesses excluded) (yes/no), and working in shifts (yes/no) were taken into account as potential confounding factors. $6,27,36,37$ Information on these factors was gathered through the questionnaires. Age and educational level were assessed at baseline T0 (May 1998), while the presence of a long-term illness and living situation were assessed in the T6 cohort questionnaire of May 2000. Information on smoking and working in shifts was present in the questionnaire T8.

\section{Statistical analysis}

Statistical analyses were performed with SPSS 13.0 and SAS. All analyses were stratified for gender. Logistic regression analyses were performed to study the cross-sectional relation between depressive complaints and sickness absence at the time of completing the questionnaire $t 8$. Because the distribution of total number of days absent from work over ten months was skewed like a Poisson distribution, Poisson regression analyses were used to test differences in number of sickness absence days between employees with different levels of depressive complaints. Multivariate survival analyses using Cox regression were conducted to examine prospective effects of depressive complaints, in which we modeled the time to first sickness absence spell from work over the ten month period following the questionnaire in January 2001. In all analyses adjustments were applied in three steps. In the first step, odds ratios or hazard ratios (ORs and HRs respectively) and $95 \%$ confidence intervals $(95 \% \mathrm{Cl}$ ) were calculated for depressive complaints adjusted for age. In the second step additional adjustments were made for the presence of a long-term illness and for smoking. In the third step, additional adjustments were made for educational level, living alone and working in shifts. Other statistical procedures included Chi-square tests and ANOVA.

\section{Results}

We first studied the relationship between the level of depressive complaints (HAD-D continuous) and sickness absence (yes/no). The average score on the HAD-D for men who were on sick leave at T8 was 6.73 (SD 4.53) and 5.84 (SD 4.45) for women. The average HAD-D score for employees not on sick leave at T8 was 3.84 (SD 3.65) for men and 3.37 (SD 3.49) for women.

Table 1 presents descriptive characteristics of the study population, categorized by the level of depressive complaints. 
Table 1 Descriptive characteristics of the study population, stratified for gender and level of depressive complaints

\begin{tabular}{|c|c|c|c|c|}
\hline & \multicolumn{4}{|l|}{$\operatorname{Men}(n=5,385)$} \\
\hline & $\begin{array}{l}\text { Normal range } \\
\text { (<8 points) }\end{array}$ & $\begin{array}{l}\text { Mild complaints } \\
\text { (8-10 points) }\end{array}$ & $\begin{array}{l}\text { Moderate } \\
\text { and severe } \\
\text { complaints } \\
(\geq 11)\end{array}$ & $\mathrm{p}$-value \\
\hline $\mathrm{N} ;(\%)^{*}$ & $4,288(81.4)$ & $612(11.6)$ & $365(6.9)$ & \\
\hline Age; Mean (SD) & 45.37 (8.34) & $47.32(7.30)$ & $46.20(7.21)$ & $<0.001$ \\
\hline Highest level of education; \% & & & & $<0.001$ \\
\hline Low & 18.9 & 26.4 & 26.6 & \\
\hline Medium & 40.4 & 43.6 & 42.3 & \\
\hline High & 40.7 & 30.1 & 31.1 & \\
\hline Long-term illness; \% & 16.3 & 28.1 & 27.1 & $<0.001$ \\
\hline Smoking; \% & 20.9 & 26.5 & 26.3 & 0.001 \\
\hline Living alone; \% & 8.1 & 8.4 & 9.9 & 0.464 \\
\hline Shift work; \% & 23.7 & 27.7 & 31.3 & 0.001 \\
\hline $\begin{array}{l}\text { Absent from work at time of } \\
\text { completing t8; \% }\end{array}$ & 4.1 & 9.7 & 17.2 & $<0.001$ \\
\hline Complete absence & 2.4 & 6.4 & 12.2 & \\
\hline \multirow[t]{3}{*}{ Partial absence } & 1.7 & 3.4 & 5.0 & \\
\hline & \multicolumn{4}{|c|}{ Women $(n=1,992)$} \\
\hline & $\begin{array}{l}\text { Normal range } \\
\text { (<8 points) }\end{array}$ & $\begin{array}{l}\text { Mild complaints } \\
\text { (8-10 points) }\end{array}$ & $\begin{array}{l}\text { Moderate } \\
\text { and severe } \\
\text { complaints } \\
(\geq 11)\end{array}$ & $\mathrm{p}$-value \\
\hline $\mathrm{N} ;(\%) \dagger$ & $1,645(84.4)$ & $184(9.4)$ & $120(6.2)$ & \\
\hline Age; Mean (SD) & $41.10(8.36)$ & $42.88(8.69)$ & $42.63(8.67)$ & 0.006 \\
\hline Highest level of education; \% & & & & 0.487 \\
\hline Low & 10.9 & 12.8 & 14.3 & \\
\hline Medium & 54.0 & 52.8 & 58.0 & \\
\hline High & 35.0 & 34.4 & 27.7 & \\
\hline Long-term illness; \% & 20.8 & 26.8 & 30.9 & 0.018 \\
\hline Smoking; \% & 23.6 & 23.9 & 29.2 & 0.382 \\
\hline Living alone; \% & 10.2 & 16.9 & 14.2 & 0.012 \\
\hline Shift work; \% & 21.0 & 17.5 & 17.4 & 0.390 \\
\hline $\begin{array}{l}\text { Absent from work at time of } \\
\text { completing t8; } \%\end{array}$ & 7.4 & 16.0 & 28.0 & $<0.001$ \\
\hline Complete absence & 4.9 & 11.0 & 21.2 & \\
\hline Partial absence & 2.5 & 5.0 & 6.8 & \\
\hline
\end{tabular}

* Row totals of cases are not equal to 5,385 due to missing values on the other variables; $†$ Row totals of cases are not equal to 1,992 due to missing values on the other variables. 
As shown in table 1, in both men and women, statistically significant differences were observed between the three categories of depressive complaints with respect to demographic, health, and work related factors. In men, all factors, except living alone differed significantly between the categories of depressive complaints. Employees reporting mild or moderate-severe complaints had a higher mean age, a higher percentage of lower educational level, and a higher proportion of long-term illness, smokers, and shift workers. In women, those reporting mild or moderate-severe complaints had a statistically significant higher mean age, a higher percentage of long-term illness and a higher proportion of employees living alone compared with employees scoring within the normal range. The proportion of employees absent from work at the time of completing the baseline was also statistically significant associated with depressive complaints in both men and women, again with those reporting mild or moderate-severe complaints having a significantly higher proportion of sickness absence at the time of completing the questionnaire T8 compared with employees scoring in the normal range.

To explore the cross-sectional associations between depressive complaints and sickness absence at the time of completing the questionnaire, we first studied the effects of one point increase on the HAD-D scale to use the full continuum of the scale. In both men and women statistically significant associations were found. In men the OR was 1.15 (95\% Cl 1.11-1.19) and in women 1.16 (95\% Cl 1.11-1.21) after adjusting for age, long-term illness, smoking, educational level, living alone and shift work.

Table 2 shows the cross-sectional association between the categories of depressive complaints and sickness absence at the time of completing the baseline questionnaire. In both men and women ORs were statistically significant and relevant for social-medical counseling. ORs increased remarkably as the level of depressive complaints increased. In women, the highest odds of sickness absence was observed among employees with moderate-severe complaints compared with those scoring within the normal range. In men, the highest odds of sickness absence was observed among employees with mild complaints compared with those scoring within the normal range. For the prospective analyses, over 10 months of follow-up, we examined the effect of depressive complaints on time to onset of first sickness absence spell, irrespective of sickness duration, based on company registered sick leave data. Again we first studied the effects of one point increase on the HAD-D scale to use the full continuum of the scale. In both men and women, a significant association between depressive complaints and the time to onset of the first sickness absence spell was found. The HR was 1.03 (1.01-1.05) in men and 1.04 (1.01-1.07) in women after adjusting for age, long-term illness, smoking, educational level, living alone and shift work. The mean time to onset of first sickness absence spell in women was 212 days for employees scoring within the normal range (SD 117.61), 190 days (SD 119.03) for those with mild complaints and 183 days (SD 118.96) for those with moderate-severe complaints. For men this was 241 days for employees scoring within the normal range (SD 105.88), 226 days for those with mild complaints (SD 114.84) and 220 days for those with moderate-severe complaints (SD 114.77). 
Table 2 Cross-sectional association between depressive complaints and sickness absence in men and women

\begin{tabular}{|c|c|c|c|c|}
\hline & \multicolumn{4}{|l|}{ Men } \\
\hline & $\mathrm{N}$ & $\mathrm{OR}^{*}(95 \% \mathrm{Cl})$ & ORt $(95 \% \mathrm{Cl})$ & OR $(95 \% \mathrm{Cl})$ \\
\hline Normal range & 3,895 & 1 & 1 & 1 \\
\hline Mild complaints & 512 & $2.58(1.83-3.65)$ & $2.35(1.66-3.33)$ & $2.21(1.56-3.15)$ \\
\hline \multirow[t]{3}{*}{ Moderate and severe complaints } & 290 & $4.59(3.18-6.62)$ & $4.24(2.93-6.14)$ & $4.00(2.76-5.81)$ \\
\hline & \multicolumn{4}{|c|}{ Women } \\
\hline & $\mathrm{N}$ & $\mathrm{OR}^{*}(95 \% \mathrm{CI})$ & ORt $(95 \% \mathrm{CI})$ & OR $\ddagger(95 \% \mathrm{Cl})$ \\
\hline Normal range & 1,395 & 1 & 1 & 1 \\
\hline Mild complaints & 153 & $1.87(1.10-3.20)$ & $1.79(1.04-3.07)$ & $1.73(1.00-2.98)$ \\
\hline Moderate and severe complaints & 89 & $5.70(3.45-9.43)$ & $5.41(3.25-9.00)$ & $5.33(3.20-8.88)$ \\
\hline
\end{tabular}

Column totals for men are not equal to 5,385 due to missing values.

Column totals for women are not equal to 1,992 due to missing values.

$O \mathrm{R}^{*}$ : adjusted for age.

ORt: additionally adjusted for long-term illness, smoking.

ORł: additionally adjusted for educational level, living alone, shift work.

Table 3 presents prospective associations between the three categories of depressive complaints and time to onset of first sickness absence spell in the ten months (March-December) following the questionnaire in January 2001. For both men and women, the HRs for the association between depressive complaints and time to onset of the first sickness absence spell were all in the expected direction, in which those with mild or moderate-severe complaints had a higher risk of going on sick leave earlier than employees scoring within the normal range. For women, only one significant and relevant association was found for those with moderate-severe complaints when adjusted for age. This is probably due to the small number of employees having mild or moderate-severe complaints among women. For men, the HRs for moderate-severe depressive complaints, adjusted for all the confounding factors, were relevant and reached statistical significance. 
Table 3 Depressive complaints as a risk factor for a shorter time to onset of first sickness absence spell

\begin{tabular}{|c|c|c|c|c|}
\hline & \multicolumn{4}{|l|}{ Men } \\
\hline & $\mathrm{n}$ & $\mathrm{HR}^{*}(95 \% \mathrm{Cl})$ & $\mathrm{HRT}(95 \% \mathrm{Cl})$ & HR $\ddagger(95 \% \mathrm{CI})$ \\
\hline Normal range & 2,098 & 1 & 1 & 1 \\
\hline Mild complaints & 332 & $1.18(0.97-1.44)$ & $1.16(0.95-1.42)$ & $1.20(0.98-1.46)$ \\
\hline \multirow[t]{3}{*}{ Moderate-severe complaints } & 188 & $1.45(1.13-1.86)$ & $1.45(1.13-1.86)$ & $1.57(1.22-2.02)$ \\
\hline & \multicolumn{4}{|c|}{ Women } \\
\hline & $\mathrm{n}$ & $\mathrm{HR}^{*}(95 \% \mathrm{Cl})$ & $\mathrm{HRT}(95 \% \mathrm{Cl})$ & HR $(95 \%$ Cl) \\
\hline Normal range & 556 & 1 & 1 & 1 \\
\hline Mild complaints & 64 & $1.38(0.95-2.02)$ & $1.33(0.91-1.94)$ & $1.36(0.93-1.99)$ \\
\hline Moderate-severe complaints & 35 & $1.63(1.04-2.55)$ & $1.53(0.98-2.41)$ & $1.56(0.99-2.45)$ \\
\hline
\end{tabular}

Column totals for men are not equal to 2,666 due to missing values.

Column totals for women are not equal to 673 due to missing values.

$H R^{*}$ : adjusted for age.

HRT: additionally adjusted for long-term illness, smoking.

HR : additionally adjusted for educational level, living alone, shift work.

To analyze duration of sickness absence, poisson regression analyses were used. Again, first the effects of one point increase on the HAD-D scale were studied. Table 4 shows statistically significant $\beta^{\prime}$ s in both men and women after adjusting for age, long-term illness, smoking, educational level, living alone and shift work. These results show a statistically significant association between depressive complaints and sickness absence duration. Similar results were found after performing the analyses with the classification into the three categories of depressive complaints (results not displayed). In both men and women, the risk of a longer duration of sickness absence increased as the level of depressive complaints increased.

Table 4 Depressive complaints (continuous) as risk factor for sickness absence duration

\begin{tabular}{|c|c|c|c|}
\hline & Model* & Modelt & Model \\
\hline Depressive complaints in: & B $(95 \% \mathrm{CI})$ & ß $(95 \% \mathrm{CI})$ & $\beta(95 \% \mathrm{CI})$ \\
\hline Men & $0.0794(0.0623-0.0965)$ & $0.0777(0.0596-0.0959)$ & $0.0735(0.0549-0.0921)$ \\
\hline Women & $0.0813(0.0479-0.1148)$ & $0.0762(0.0416-0.1108)$ & $0.0730(0.0366-0.1095)$ \\
\hline
\end{tabular}

Model $^{*}$ : adjusted for age.

Modelt: additionally adjusted for long-term illness and smoking.

Modelf: additionally adjusted for educational level, living alone and shift work. 
Table 5 Average number of days absent from work over ten months of follow-up among the three categories of depressive complaints

\begin{tabular}{|c|c|c|c|c|}
\hline & \multicolumn{4}{|l|}{ Men } \\
\hline & $\mathrm{n}$ & Mean & $95 \% \mathrm{Cl}$ & $\mathrm{p}$-value \\
\hline Normal range & 2,098 & 7.67 & $7.02-8.38$ & - \\
\hline Mild complaints & 332 & 14.48 & $12.31-17.03$ & $<0.001$ \\
\hline \multirow[t]{3}{*}{ Moderate-severe complaints } & 188 & 17.79 & 14.67-21.57 & $<0.001$ \\
\hline & \multicolumn{4}{|c|}{ Women } \\
\hline & $\mathrm{n}$ & Mean & $95 \% \mathrm{Cl}$ & $\mathrm{p}$-value \\
\hline Normal range & 556 & 11.01 & $9.39-12.92$ & - \\
\hline Mild complaints & 64 & 27.37 & $20.32-36.88$ & $<0.001$ \\
\hline Moderate-severe complaints & 35 & 19.51 & $12.34-30.86$ & 0.078 \\
\hline
\end{tabular}

Column totals for men are not equal to 2,666 due to missing values.

Column totals for women are not equal to 673 due to missing values.

Translated to daily practice the higher risk for a longer duration of sickness absence revealed itself in the mean number of absent days in the three categories of depressive complaints, as is shown in table 5 . This table shows substantial and relevant differences in the average number of absent days from work over ten months of follow-up among employees with different levels of depressive complaints. The average number of absent days between the different levels of depressive complaints was statistically significant, except in women with moderate-severe complaints. This may be due to the low power in this subgroup. The results were most pronounced for women with mild depressive complaints. The average number of sickness absence days over ten months of follow-up was almost 2.5 times as high as the average number of sickness absence days for employees scoring in the normal range.

\section{Discussion}

Although several studies investigated and found an association between major depression and sickness absence, this study has shown clear cross-sectional and prospective relations between levels of depressive complaints and future sickness absence, even after adjusting for several important confounding factors. Hence, this study suggests that also less severe levels of depressive complaints constitute risk factors for sickness absence, which is an important finding for the development of preventive measures with respect to sickness absence.

Because cross-sectional associations do not allow assertions on the specific causality of associations between depressive complaints and sickness absence, the prospective analyses suggest that depressive complaints can be considered as a predictor of sickness absence. In particular, the cross-sectional associations were very pronounced. This may indicate that a large part of the effect of depressive complaints on sickness absence may already have been present at the baseline measurement (time 
of completing questionnaire T8). Because of these high cross-sectional associations, the results from the longitudinal analyses may have been an underestimation.

In 2002, the annual total sickness absence rate in the general Dutch population was $4.8 \%$ in men and $6.0 \%$ in women. ${ }^{38}$ The percentages of employees scoring in the normal range of depressive complaints and who were absent from work at the time of completing T8 are fairly consistent with these data, $4.1 \%$ for men and $7.4 \%$ for women. In men, $17.2 \%$ of the employees with moderatesevere complaints and $9.7 \%$ of the employees with mild depressive complaints were absent from work at the time of completing the questionnaire T8. In women, this was $28.0 \%$ of the employees with moderate-severe complaints and $16.0 \%$ of the employees with mild depressive complaints. Firm conclusions about gender differences in the relationship between depressive complaints and sickness absence cannot be drawn though, partly because of the small number of women especially in the mild and moderate-severe complaints groups.

Although a reasonable association was found with respect to level of depressive complaints and time to onset of first sickness absence spell, pronounced differences between employees with a higher level versus a lower level of depressive complaints were observed with respect to the average number of days absent over the ten months follow-up period. It appears that only employees with moderate-severe depressive complaints report themselves ill at an earlier point in time. The duration of sickness absence however, increases the most when employees with mild complaints and employees scoring within the normal range were compared, with twice as many absent days in men and two and a halve times more in women. This provides an indication that sickness absence as a consequence of depressive complaints occurs earlier when a person already experiences more severe complaints. For instance, when a person with mild depressive complaints does decide to go on sick leave, the duration of sickness absence increases significantly compared with the sickness absence duration of employees scoring within the normal range. Employees with mild complaints may be able to continue their work much longer without experiencing considerable restraints or they might not be aware of their psychological complaints when they first appear compared with employees with moderate-severe complaints.

In this study, depressive complaints were measured with a questionnaire instead of using a diagnostic interview. The Hospital Anxiety and Depression (HAD-D) scale was used to measure depressive complaints in the working population and to identify employees with different severity levels of depressive complaints. Bjelland et al. ${ }^{34}$ found that the HAD-D performed well in assessing the severity and caseness of anxiety disorders and depression in the general population. Less is known about application of the HAD-D in the working population. Although based on self-report, Andrea et al. showed that the HAD-D and HAD-A can be used as separate constructs to measure depressive complaints and anxiety in the working population ${ }^{5}$ and it is therefore sufficient to use the HAD-D in this study only. The surplus value of using the HAD-scale in our study is that it enabled us to investigate both the whole continuum and the distinguished categories of depressive complaints.

The prevalence of employees with moderate-severe depressive complaints in our study was $6.9 \%$ for men and $6.2 \%$ for women. The prevalence for men in our study population seems comparable with 
other studies based on DSM-III-R classifications which present annual depression prevalences for men and women separately. ${ }^{5}$ However, the prevalence of moderate-severe depressive complaints for women in our study seems to be lower. This lower prevalence may be caused by the fact that more women than men work part-time and less women work in shifts. Thus, they may have already adapted their work to their health status and/or private situation. Another explanation may be that women with mild or moderate-severe depressive complaints have already left the labor force at the time the questionnaire was distributed.

In the analyses, several adjustments were made to investigate whether the effects of depressive complaints on sickness absence could be ascribed to depressive complaints or should be attributed to other confounding factors, known to be associated with sickness absence. Therefore, we adjusted the analyses in three steps for several factors. Although the ORs in the cross-sectional analyses were reduced after controlling for these factors, similar trends generally remained. In the prospective analyses, small changes in observed effects also occurred after controlling for these confounding factors. The observed effects on sickness absence after controlling for confounding factors could then more likely be ascribed to depressive complaints. Although the strength of the associations was hardly reduced after controlling for the selected group of confounding factors, it could be argued that there may be other important risk factors which contribute to the effects found, given the multi-factorial etiology of sickness absence. ${ }^{27}$ Due to practical limitations data on all potential confounding factors were not available in the questionnaire. For this reason, it is important to control for educational level, as it is often viewed as a proxy for other factors related to occupation or lifestyle. ${ }^{39}$

The results of this study are based on data from a large scale prospective cohort study, enabling us to study the cross-sectional and prospective relationship between levels of depressive complaints and long-term sickness absence among employees over a ten month follow-up period. Sickness absence was measured through linkage on an individual level with the company records on sickness absence, providing us with objective sickness absence data. A time lag of two months was used between the time employees received the questionnaire and the measurement of sickness absence, as the time of the returning of the questionnaires and the processing of the data would take a maximum of two months. For the prospective analyses, it may be possible however, that respondents were already on sick leave because of depressive complaints before the measuring of sickness absence began. This means there might be a shorter time between depressive complaints and occurrence of sickness absence as a consequence than our predefined two months lag-time. These persons were excluded from the analyses, because prevalent cases at the start of the measurement were excluded. Therefore, the effect of depressive complaints on sickness absence could have been an underestimation.

The baseline population of the present study consists of participants of the Maastricht Cohort Study after two years of follow-up. This might raise questions about selective drop-out of participants during follow-up. Analyses on the effect of non-response during follow-up with respect to sickness absence indicated that employees reporting themselves sick four or more times showed a lower response rate on the questionnaires as compared with those reporting no sickness absence or calling 
in sick once or twice. This results in an underestimation of the incidence of sick leave.$^{40}$ For our study, this might also indicate an underestimation of sickness absence.

Sickness absence data that were derived from the questionnaire were used only for the crosssectional analyses. Although measured by self-report, we argue that no recall bias has occurred since workers were asked for sickness absence at the time of completing the questionnaire.

From the present study, we conclude that a clear relationship exists between depressive complaints and sickness absence. The high cross-sectional association at baseline measurement and the prospective association between depressive complaints and sickness absence indicate that the effects of mild or moderate-severe depressive complaints on sickness absence may occur rather quickly over time, resulting in a shorter time to onset of sickness absence and much longer periods of sickness absence. Because of this and the high prevalence of mild and moderate-severe depressive complaints in the labor force, prevention of depressive complaints might be beneficial in preventing future sickness absence.

\section{Acknowledgements}

The Maastricht Cohort Study is part of the Netherlands concerted research action on 'Fatigue at Work' granted by the Netherlands Organization for Scientific Research (NWO). This study was supported by the Netherlands Organization for Health Research and Development (Zorg Onderzoek Nederland, grant no. 62200024), and by CAPHRI School for Public Health and Primary Care, Maastricht, the Netherlands. 


\section{References}

1. World Health Organization. World Health Report, Geneva. 2000.

2. Bijl RV, Ravelli A, van Zessen G. Prevalence of psychiatric disorder in the general population: results of The Netherlands Mental Health Survey and Incidence Study (NEMESIS). Soc Psychiatry Psychiatr Epidemiol. 1998;33:587-95.

3. Cuijpers $P$, de Graaf R, van Dorsselaer S. Minor depression: risk profiles, functional disability, health care use and risk of developing major depression. J Affect Disord. 2004;79:71-9.

4. Statistics Netherlands. Zelfgerapporteerde medische consumptie, gezondheid en leefstijl. [Self-reported medical consumption, health and lifestyle] Heerlen/Voorburg, the Netherlands. Available at http://www. statline.cbs.nl Accessed 20 June 2008.

5. Andrea H, Bultmann U, Beurskens AJ, Swaen GM, van Schayck CP, Kant IJ. Anxiety and depression in the working population using the HAD Scale--psychometrics, prevalence and relationships with psychosocial work characteristics. Soc Psychiatry Psychiatr Epidemiol. 2004;39:637-46.

6. Lerner D, Henke RM. What does research tell us about depression, job performance, and work productivity? J Occup Environ Med. 2008;50:401-10.

7. Jenkins R. Mental illness and work. In: Floyd M, Povall M, Watson G, eds. Mental health at work. London: Jessica Kingsley; 1994:77-9.

8. Simon GE, Chisholm D, Treglia M, Bushnell D. Course of depression, health services costs, and work productivity in an international primary care study. Gen Hosp Psychiatry. 2002;24:328-35.

9. Smit F, Beekman A, Cuijpers P, de Graaf R, Vollebergh W. Selecting key variables for depression prevention: results from a population-based prospective epidemiological study. J Affect Disord. 2004;81:241-9.

10. Bultmann U, Rugulies R, Lund T, Christensen KB, Labriola M, Burr H. Depressive symptoms and the risk of long-term sickness absence: a prospective study among 4747 employees in Denmark. Soc Psychiatry Psychiatr Epidemiol. 2006;41:875-80.

11. Kessler RC, Barber C, Birnbaum HG, Frank RG, Greenberg PE, Rose RM, et al. Depression in the workplace: effects on short-term disability. Health Aff (Millwood). 1999;18:163-71.

12. Nieuwenhuijsen K, Verbeek JH, de Boer AG, Blonk RW, van Dijk FJ. Predicting the duration of sickness absence for patients with common mental disorders in occupational health care. Scand J Work Environ Health. 2006;32:67-74.

13. Stansfeld S, Feeney A, Head J, Canner R, North F, Marmot M. Sickness absence for psychiatric illness: the Whitehall II Study. Soc Sci Med. 1995;40:189-97.

14. Laitinen-Krispijn S, Bijl RV. Mental disorders and employee sickness absence: the NEMESIS study. Netherlands Mental Health Survey and Incidence Study. Soc Psychiatry Psychiatr Epidemiol. 2000;35:71-7.

15. Lerner D, Adler DA, Chang H, Lapitsky L, Hood MY, Perissinotto C, et al. Unemployment, job retention, and productivity loss among employees with depression. Psychiatr Serv. 2004;55:1371-8.

16. Hensing G, Alexanderson K, Akerlind I, Bjurulf P. Sick-leave due to minor psychiatric morbidity: role of sex integration. Soc Psychiatry Psychiatr Epidemiol. 1995;30:39-43.

17. Kivimaki M, Vahtera J, Thomson L, Griffiths A, Cox T, Pentti J. Psychosocial factors predicting employee 
sickness absence during economic decline. J Appl Psychol. 1997;82:858-72.

18. Laaksonen $\mathrm{M}$, Martikainen $\mathrm{P}$, Rahkonen $\mathrm{O}$, Lahelma E. Explanations for gender differences in sickness absence: evidence from middle-aged municipal employees from Finland. Occup Environ Med. 2008;65:32530.

19. Kopp MS, Skrabski A, Szedmak S. Socioeconomic factors, severity of depressive symptomatology, and sickness absence rate in the Hungarian population. J Psychosom Res. 1995;39:1019-29.

20. Koopmans PC, Roelen CA, Groothoff JW. Sickness absence due to depressive symptoms. Int Arch Occup Environ Health. 2008;81:711-9.

21. North F, Syme SL, Feeney A, Head J, Shipley MJ, Marmot MG. Explaining socioeconomic differences in sickness absence: the Whitehall II Study. BMJ. 1993;306:361-6.

22. Feeney A, North F, Head J, Canner R, Marmot M. Socioeconomic and sex differentials in reason for sickness absence from the Whitehall II Study. Occup Environ Med. 1998;55:91-8.

23. Hensing G, Alexanderson K, Allebeck P, Bjurulf P. Sick-leave due to psychiatric disorder: higher incidence among women and longer duration for men. Br J Psychiatry. 1996;169:740-6.

24. Hardy GE, Woods D, Wall TD. The impact of psychological distress on absence from work. J Appl Psychol. 2003;88:306-14.

25. Spijker J, de Graaf R, Bijl RV, Beekman AT, Ormel J, Nolen WA. Duration of major depressive episodes in the general population: results from The Netherlands Mental Health Survey and Incidence Study (NEMESIS). Br J Psychiatry. 2002;181:208-13.

26. Vaananen A, Toppinen-Tanner S, Kalimo R, Mutanen P, Vahtera J, Peiro JM. Job characteristics, physical and psychological symptoms, and social support as antecedents of sickness absence among men and women in the private industrial sector. Soc Sci Med. 2003;57:807-24.

27. Alexanderson K. Sickness absence: a review of performed studies with focused on levels of exposures and theories utilized. Scand J Soc Med. 1998;26:241-9.

28. Janssen N, Kant IJ, Swaen GM, Janssen PP, Schroer CA. Fatigue as a predictor of sickness absence: results from the Maastricht cohort study on fatigue at work. Occup Environ Med. 2003;60:71-6.

29. Kessler RC, Akiskal HS, Ames M, Birnbaum H, Greenberg P, Hirschfeld RM, et al. Prevalence and effects of mood disorders on work performance in a nationally representative sample of U.S. workers. Am J Psychiatry. 2006;163:1561-8.

30. Kessler RC, Frank RG. The impact of psychiatric disorders on work loss days. Psychol Med. 1997;27:861-73.

31. Lerner D, Adler DA, Chang H, Berndt ER, Irish JT, Lapitsky L, et al. The clinical and occupational correlates of work productivity loss among employed patients with depression. J Occup Environ Med. 2004;46:46-55.

32. Kant IJ, Bultmann U, Schroer KA, Beurskens AJ, Van Amelsvoort LG, Swaen GM. An epidemiological approach to study fatigue in the working population: the Maastricht Cohort Study. Occup Environ Med. 2003;60:329.

33. Zigmond AS, Snaith RP. The hospital anxiety and depression scale. Acta Psychiatr Scand. 1983;67:361-70.

34. Bjelland I, Dahl AA, Haug TT, Neckelmann D. The validity of the Hospital Anxiety and Depression Scale. An updated literature review. J Psychosom Res. 2002;52:69-77.

35. Snaith RP. The Hospital Anxiety And Depression Scale. Health Qual Life Outcomes. 2003;1:29. 
36. Allebeck P, Mastekaasa A. Swedish Council on Technology Assessment in Health Care (SBU). Chapter 3. Causes of sickness absence: research approaches and explanatory models. Scand J Public Health Suppl. 2004;63:36-43.

37. Allebeck P, Mastekaasa A. Swedish Council on Technology Assessment in Health Care (SBU). Chapter 5. Risk factors for sick leave - general studies. Scand J Public Health Suppl. 2004;63:49-108.

38. Statistics Netherlands. National statistics on sick leave, frequency, period of absence. Heerlen/Voorburg, the Netherlands. Available at http://www.statline.cbs.nl Accessed at 11 June 2008.

39. Statistics Netherlands. Standaard Beroepenclassificatie 1992 [The Netherlands Standard Classification of Occupations 1992]. 's-Gravenhage: SDU/uitgeverij; 1993.

40. Mohren D, Jansen N, Amelsvoort L, Kant IJ. An Epidemiological Approach of Fatigue and Work: Experiences from the Maastricht Cohort Study. Maastricht: Programma Epidemiologie van Arbeid en Gezondheid; 2007:78-9. 


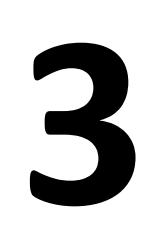

\title{
Prediction of long-term sickness absence among employees with depressive complaints
}

\author{
Monique A.S. Lexis $^{1}$ \\ Nicole W.H. Jansen ${ }^{1}$ \\ Ludovic G.P.M. van Amelsvoort ${ }^{1}$ \\ Marcus J.H. Huibers ${ }^{2}$ \\ Ate Berkouwer ${ }^{3}$ \\ Gladys Tjin A Ton ${ }^{3}$ \\ Piet A. van den Brandt ${ }^{1}$ \\ IJmert Kant ${ }^{1}$
}

${ }^{1}$ CAPHRI School for Public Health and Primary Care, Department of Epidemiology, Maastricht University, Maastricht, The Netherlands

${ }^{2}$ Department of Clinical Psychological Science, Maastricht University, Maastricht, The Netherlands ${ }^{3}$ Occupational Health Services Beter, Amsterdam, The Netherlands 


\section{Abstract}

\section{Objective}

To study the properties of a screening instrument in predicting long-term sickness absence among employees with depressive complaints.

\section{Methods}

Employees at high risk of future sickness absence were selected by the screening instrument Balansmeter (BM). Depressive complaints were assessed with the depression scale of the Hospital Anxiety and Depression Scale (HAD-D). The total study population consisted of 7,401 employees. Sickness absence was assessed objectively and analyzed at 12 and 18 months of follow-up using company registers on certified sick leave.

\section{Results}

The RR for long-term sickness absence, for employees at high risk versus not at high risk, was 3.26 (95\% Cl 2.54-4.22) in men and 2.55 (1.98-3.35) in women, when the BM was applied in the total study population. The RR of long-term sickness absence of employees with depressive complaints compared with employees without depressive complaints was 3.13 (2.41-4.09) in men and 2.45 (2.00-3.00) in women. The RR of long-term sickness absence for the BM applied in employees with depressive complaints was 5.23 in men and 3.87 in women. When the BM with a cut-off point with a higher sensitivity was applied in employees with depressive complaints, the RR was 4.88 in men and 3.80 in women.

\section{Conclusions}

The screening instrument Balansmeter is able to predict long-term sickness absence in employees with depressive complaints. The predictive properties of the BM proved better in employees with depressive complaints compared with employees of a general working population. 


\section{Introduction}

Sickness absence constitutes a considerable public health problem in Western societies, with important consequences for individuals, their families, workplaces and society. ${ }^{1}$ Within most highincome countries mental health complaints are highly prevalent, and one of the leading causes of certified sick leave. ${ }^{2,3}$ Mental health complaints are associated with prolonged sickness absence and permanent work disability. ${ }^{3-7}$ In the Netherlands, psychological health complaints accounted for the highest proportion (19\%) of total sickness absence days in 2009 , due to the high mean number of sickness absence days (approximately nine weeks) per sickness absence spell. ${ }^{8}$ Long-term sickness absence is associated with a reduced probability of returning to work. Especially rehabilitation in case of absenteeism due to psychological complaints remains difficult and does not necessarily lead to work resumption. ${ }^{9-12}$ Therefore, a preventive strategy aimed at early identification of employees at risk before sickness absence occurs may be more effective. A prerequisite for such a preventive strategy is the ability to identify employees at high risk of long-term sickness absence, so before sickness absence actually occurs. In previous research, Kant et al. ${ }^{13}$ demonstrated that it is possible to identify employees at high risk of future sickness absence using a new developed screening instrument, in Dutch called the Balansmeter. Furthermore, a randomized controlled trial demonstrated that early intervention among this population proved successful in preventing future sickness absence. ${ }^{14}$ However, both studies were conducted among a general working population with no specific complaints, and early interventions therefore included a wide range of treatments commonly used in occupational health care. Recent studies showed that depressive complaints constitute a substantial part of the mental health complaints in the working population. ${ }^{2,15}$ Additionally, Kant et al. ${ }^{14}$ found that the majority of mental health complaints consisted of depressive complaints. Besides major depression, milder levels of depressive complaints were found to be strongly associated with future long-term sickness absence as well. ${ }^{16,17}$ It was therefore hypothesized that a preventive strategy aimed at a more specific target population - that is employees identified both at high risk of future sickness absence and with depressive complaints - would be even more effective in preventing future long-term sickness absence.

For this specific population the prerequisite for a preventive strategy also is the ability to predict long-term sickness absence. However, so far little research has been done with respect to screening of future sickness absence in populations with specific complaints. The main aim of the present study therefore was to assess whether the screening instrument Balansmeter (BM) can be used to adequately identify employees at high risk of future sickness absence among employees with depressive complaints. For this assessment, first the sensitivity and specificity were calculated when the BM was applied in the total study population. Employees with depressive complaints were selected by means of the depression scale of the Hospital Anxiety and Depression Scale (HAD-D). Since depressive complaints are known to be associated with long-term sickness absence, the risk of long-term sickness absence of employees with depressive complaints versus employees without depressive complaints was calculated. Subsequently, the predictive properties of the BM 
within employees with depressive complaints were studied. Finally, the predictive properties were calculated when a cut-off point with a higher sensitivity on the BM was used, to examine the effect of having a lower risk of future sickness absence on the predictive properties of the BM.

\section{Methods}

\section{Study population and procedure}

The study was conducted among employees working at a large banking company in the Netherlands. The screening procedure examined in this study was the basis for a randomized controlled trial (RCT). The RCT aimed to examine the efficacy of a preventive intervention for future sickness absence and major depression. ${ }^{18}$ For the current study, data from the screening questionnaires were used. In 2007 , a total of 23,973 employees received the screening instrument at their home address. In the invitation letter, employees were asked to provide written informed consent, which covered the usage of the questionnaire data and the company data on sickness absence. Four separate batches were necessary to realize smooth processing of the large amount of questionnaires and to guarantee smooth enrolment of participants in the trial. Screening questionnaires were sent out in February $(n=7,000)$, March $(n=4,500)$, September $(n=6,196)$ and October $(n=6,277)$ 2007. In total, 9,157 employees (38.2\%) responded to the questionnaire. For the analyses, 8,892 questionnaires were suitable and 265 questionnaires were discarded due to technical reasons or because no informed consent was provided. Questionnaires with missing data on the Balansmeter or the HAD-D were excluded ( $n=747$ ). Further exclusion criteria for the study were being (fully or partially) absent from work, being pregnant or being on pregnancy/maternity leave, or receiving treatment by a psychologist at the time of completing the questionnaire $(n=744)$. After exclusion the total study population consisted of 7,401 employees.

\section{Measurements}

\section{Risk of future sickness absence}

The screening questionnaire, called Balansmeter (BM) in Dutch, originally developed for employees working in an office work environment, was used to identify employees at high risk of future longterm sickness absence due to any cause. The BM was developed and internally validated on data from the Maastricht Cohort Study on Fatigue at Work in 1998, and externally validated in 2003 on a large sample of employees of the same banking company the current study was conducted at. ${ }^{13}$ The BM consists of items representing risk factors for sickness absence, such as demographic factors, workrelated factors, and private-situation-related factors, that in many different combinations determine the risk of future sickness absence. Different cut-off points were used for men and women, since the prediction of sickness absence differed between men and women. In the cohort study in which 
the BM was developed, several cut-off points were determined. The original cut-off point (BM-O) resulted in a specificity of $94.7 \%$ and sensitivity of $30.3 \%$ in women. In men, specificity was $94.3 \%$ and sensitivity $49.4 \%$. The low cut-off point of the BM (BM-L) resulted in a specificity of $87.9 \%$ and sensitivity of $52.6 \%$ in women. In men, specificity was $87.8 \%$ and sensitivity was $65.1 \%$.

\section{Depressive complaints}

The Hospital Anxiety and Depression (HAD) scale was used to measure the presence and severity of depressive complaints. The HAD is a 14-item self-report questionnaire, originally developed to indicate the presence and severity of both anxiety (HAD-A) and depression (HAD-D) separately. ${ }^{19,20}$ Both HAD-A and HAD-D consist of 7 items and are scored on a four point Likert scale, resulting in a range of 0 to 21. In this study only the HAD-D was used. With respect to the cut-off values, we used the values suggested by Zigmond et al. ${ }^{20}$ and confirmed by a review from Bjelland et al. ${ }^{21} \mathrm{~A}$ score on the HAD-D of 8 points or higher indicates mild to severe depressive complaints. The cut-off point of 8 represents a sensitivity and specificity of approximately $80 \% .^{21}$

\section{Demographics and health-related factors}

Employees provided information on gender, age, educational level (low, medium or high) and presence of a long-term illness (defined as having the disease for more than six months) through self-report in the screening questionnaire.

\section{Outcome measure}

\section{Sickness absence}

Sickness absence was measured objectively through record linkage on an individual level with the company registers on certified sickness absence from two months after the employees received the screening instrument until 20 months of follow-up, resulting in an 18 month follow-up period. A time lag of two months was maintained, which is in line with the development of the Balansmeter. ${ }^{13}$ Longterm sickness absence was defined as a sickness absence spell lasting for more than 28 consecutive calendar days. Self-reported sickness absence data were used for exclusion of prevalent cases of sickness absence.

\section{Statistical analyses}

For each respondent sum scores on the BM were calculated. Based on these sum scores, employees at high risk of future sickness absence (cases, scoring above the cut-off point) and employees not at high risk of future sickness absence (non-cases, scoring below the cut-off point) were identified. Depressive complaints were identified using the questionnaire HAD-D. Employees who scored 8 points or more on HAD-D were defined as having mild to severe depressive complaints (cases). The association between cases and non-cases and the outcome long-term sickness absence (> 28 
consecutive calendar days) was examined by calculating relative risks. Analyses were performed for 12 and 18 months of follow-up and were performed for women and men separately, since the prediction of sickness absence by the BM differed for men and women. Gender differences in depressive complaints and sickness absence are often mentioned in the literature as well. ${ }^{22-25}$ The associations were calculated for four situations:

1 the BM (BM-O) applied in the total study population;

2 the HAD-D applied in the total study population;

3 the BM (BM-O) applied in employees with depressive complaints (HAD-D) within the total study population;

4 the BM with the adapted cut-off point (BM-L), in employees with depressive complaints (HAD-D) within the total study population.

To study these associations, data from a RCT were used. ${ }^{18}$ The RCT included an intervention that aimed at prevention of long-term sickness absence. The intervention group had to be taken into account when performing the analyses, since the intervention was expected to have resulted in decreased long-term sickness absence. Therefore, recalculations were performed for each of the four situations for participants who received the intervention, to minimize the influence of the results of the intervention group on the results of this study. For each situation, the number of employees who were part of the intervention group was calculated among the cases and non-cases of BM (situation 1, 3 and 4) and HAD-D (situation 2), and their proportion of long-term sickness absence was replaced by the proportion of sickness absence of the control group. The intervention and control group of the RCT originated from the same population, and since participants were randomly allocated to the intervention and control group, these groups could be considered comparable. In case the intervention group would not have received an intervention, the proportion of long-term sickness absence was expected to be equal in the intervention and control group. Therefore, the proportion of long-term sickness absence of the employees of the intervention group was not included in the study, but replaced by the proportion of sickness absence of the control group.

With regard to the non-cases, they were not part of the RCT and as such all non-cases were included in the present study. However, employees who scored above one of two cut-off points and who reported high scores on the BM or on the HAD-D were referred to an occupational physician. Information on treatment received by these employees was collected. Approximately $2 \%$ of these employees received some kind of treatment. Hence, the contrast in sickness absence between cases and non-cases was not likely to have been reduced by the treatment.

Stata statistical software package 8.0 was used for preparation of the objective sickness absence data. Analyses were performed using SPSS 15.0. 


\section{Results}

Table 1 presents the descriptive characteristics of the total study population $(N=7,401)$, for men and women separately. Table 1 shows that although the study was conducted in a single company consisting of white-collar workers, the study sample represents a rather heterogeneous population with respect to age, educational level, presence of long-term illness, depressive complaints, sickness absence and having an executive function.

Table 1 Descriptive characteristics of the total study population $(\mathrm{N}=7,401)$

\begin{tabular}{|c|c|c|}
\hline Variable & $\begin{array}{l}\text { Men } \\
(n=3,858)\end{array}$ & $\begin{array}{l}\text { Women } \\
(n=3,543)\end{array}$ \\
\hline Age (18-65); mean (SD) & $45.07(9.95)$ & $40.13(8.68)$ \\
\hline \multicolumn{3}{|l|}{ Highest level of education; $\mathrm{N}(\%)^{*}$} \\
\hline Low & $66(1.8)$ & $118(3.5)$ \\
\hline Medium & $1,804(49.1)$ & $2,174(64.4)$ \\
\hline High & $1,807(49.1)$ १ & $1,084(32.1)$ ๆ \\
\hline Presence of long-term illness; $\mathrm{N}(\%) \dagger$ & $886(23.5)$ & $860(24.8)$ \\
\hline \multicolumn{3}{|l|}{ Employees at high risk of future sickness absence } \\
\hline (\% scoring above the original cut-off point of the Balansmeter) & 10.1 & 3.9 \\
\hline \multicolumn{3}{|l|}{ Depressive complaints (HAD-D) (0-21) $\neq ;$} \\
\hline Continuous score; mean (SD) & $2.87(3.04)$ & $2.75(3.18)$ \\
\hline $\mathrm{HAD}-\mathrm{D} \geq 8 ; \mathrm{N}(\%)$ & $353(9.1)$ & $312(8.8)$ \\
\hline \multicolumn{3}{|l|}{ Number of absence days (calendar days); mean (SD) } \\
\hline $0-12 * *$ & $17.77(38.23)$ & $23.81(46.42)$ \\
\hline $0-18 \S$ & $23.00(48.77)$ & $32.62(62.91)$ \\
\hline Having an executive function; $\mathrm{N}(\%) \dagger$ & $1,061(27.5)$ & $477(13.5)$ \\
\hline
\end{tabular}

Table 2 presents the predictive properties of the Balansmeter in the total study population and among employees with depressive complaints, for men and women separately, for 18 months of follow-up. In general, relatively more men than women were at high risk of future sickness absence. Besides, a rather low sensitivity and high specificity was calculated in each of the four situations. The latter indicated that most of the employees were correctly defined as being not at high risk.

As presented in table 2, first, the predictive properties of the BM-O were examined in the total study population $(\mathrm{N}=7,401)$. Of the 3,858 men, 389 men were identified at high risk of future sickness absence. Of these 389 men, an estimated number of 68.6 male cases experienced long-term sickness 
Table 2 Predictive properties, sensitivity and specificity of the Balansmeter (BM-O, BM-L) applied in the total study population and in employees with depressive complaints for 18 months of follow-up

\begin{tabular}{|c|c|c|c|c|c|}
\hline & Men & & & & \\
\hline & $\begin{array}{l}\text { Cases } \\
(n, \%)\end{array}$ & $\begin{array}{l}\text { Proportion } \\
\text { long-term } \\
\text { sickness } \\
\text { absence in } \\
\text { cases } \\
(\mathrm{n}, \%)^{*}\end{array}$ & $\begin{array}{l}\text { Sensitivity } \\
(\%)\end{array}$ & $\begin{array}{l}\text { Specificity } \\
(\%)\end{array}$ & $\begin{array}{l}\text { Long-term } \\
\text { sickness } \\
\text { absence } \\
\text { (> } 28 \text { calendar } \\
\text { days); } \\
\text { Relative Risks } \\
(95 \% \text { Cl) }\end{array}$ \\
\hline $\begin{array}{l}1 \mathrm{BM}(\mathrm{BM}-0) \text { in total study population } \\
(\mathrm{n}=3,858)\end{array}$ & $389(10.1)$ & $68.6(17.6)$ & 26.8 & 91.1 & $3.26(2.54-4.22)$ \\
\hline 2 HAD-D in total population $(\mathrm{n}=3,858)$ & $353(9.1)$ & $61.9(17.5)$ & 24.0 & 91.9 & $3.13(2.41-4.09)$ \\
\hline $\begin{array}{l}3 \text { BM (BM-0) in employees with } \\
\text { depressive complaints } \\
(\text { HAD-D >8) (n=353) }\end{array}$ & $93(26.3)$ & $22.7(24.4)$ & 37.4 & 75.9 & $1.67(1.07-2.68)$ \\
\hline \multirow{3}{*}{$\begin{array}{l}4 \mathrm{BM}(\mathrm{BM}-\mathrm{L}) \text { in employees with } \\
\text { depressive complaints } \\
(\mathrm{HAD}-\mathrm{D}>8)(\mathrm{n}=353)\end{array}$} & $132(37.4)$ & $29.8(22.6)$ & 48.2 & 64.9 & $1.56(1.00-2.46)$ \\
\hline & Women & & & & \\
\hline & $\begin{array}{l}\text { Cases } \\
(n, \%)\end{array}$ & $\begin{array}{l}\text { Proportion } \\
\text { long-term } \\
\text { sickness } \\
\text { absence in } \\
\text { cases } \\
(\mathrm{n}, \%)^{*}\end{array}$ & $\begin{array}{l}\text { Sensitivity } \\
(\%)\end{array}$ & $\begin{array}{l}\text { Specificity } \\
(\%)\end{array}$ & $\begin{array}{l}\text { Long-term } \\
\text { sickness } \\
\text { absence } \\
\text { (> } 28 \text { calendar } \\
\text { days); } \\
\text { Relative Risks } \\
(95 \% \text { Cl) }\end{array}$ \\
\hline $\begin{array}{l}1 \text { BM (BM-0) in total study population } \\
(\mathrm{n}=3,543)\end{array}$ & $138(3.9)$ & $42.6(30.9)$ & 9.4 & 96.9 & $2.55(1.98-3.35)$ \\
\hline 2 HAD-D in total population $(n=3,543)$ & $312(8.8)$ & $87.1(27.9)$ & 19.1 & 92.7 & $2.45(2.00-3.00)$ \\
\hline $\begin{array}{l}3 \text { BM (BM-0) in employees with } \\
\text { depressive complaints } \\
(\text { HAD-D >8) }(n=312)\end{array}$ & $48(15.4)$ & $19.5(40.6)$ & 22.4 & 87.3 & $1.58(1.09-2.40)$ \\
\hline $\begin{array}{l}4 \mathrm{BM}(\mathrm{BM}-\mathrm{L}) \text { in employees with } \\
\text { depressive complaints } \\
(\mathrm{HAD}-\mathrm{D}>8)(\mathrm{n}=312)\end{array}$ & $79(25.3)$ & $30.0(38.0)$ & 34.5 & 78.2 & $1.55(1.08-2.23)$ \\
\hline
\end{tabular}

* Due to recalculations proportions with fractional numbers were found.

absence. A RR of 3.26 (95\% Cl 2.54-4.22) was found. For women, 138 of the 3,543 employees were identified at high risk, and of these, 42.6 estimated cases went on long-term sickness absence. A RR of 2.55 (1.98-3.35) was found. 
Second, the predictive properties of having depressive complaints compared with having no depressive complaints were studied (HAD-D $\geq 8$ versus HAD-D $<8$ ), since depressive complaints are known to be predictive of future sickness absence. A RR of 3.13 (2.41-4.09) was found for men, and a RR of 2.45 (2.00-3.00) for women.

Third, the RR of long-term sickness absence was examined when the BM-O was applied in employees with depressive complaints. Of the 353 men, 93 cases were identified. Of the 93 cases, 227 estimated cases had long-term sickness absence. Of the 312 women, 48 cases were identified and 19.5 estimated cases went on long-term sickness absence. An additional RR of 1.67 (1.07-2.68) was found in men. This resulted in a total combined RR of long-term sickness of 5.23 (3.13 x 1.67). In women, an additional RR of 1.58 was found, resulting in a total combined RR of 3.87 ( $2.45 \times 1.58)$. The combined RRs of the BM-O applied in employees with depressive complaints were higher than the RRs of the BM-O applied in the total study population.

Fourth, the RR of long-term sickness absence was examined when the BM-L (lower cut-off point) was applied in employees with depressive complaints. In the 353 men, 132 cases were identified and an estimated number of 29.8 of the cases experienced long-term sickness absence. Of the 312 female employees with depressive complaints, 79 cases were identified and 30 of these cases had long-term sickness absence. The additional RR in men was $1.56(1.00-2.46)$ and the total combined RR was 4.88 $(3.13 \times 1.56)$. For women, the additional RR was $1.55(1.08-2.23)$ and the total combined risk was 3.80 (2.45 $\times 1.55)$. The combined RRs of the BM-L applied in employees with depressive complaints were still higher as compared with the RRs of the BM-O applied in the total study population.

The use of the lower cut-off point hardly influenced the RR of the BM-L. However, a decrease in specificity of $11.0 \%$ in men and $9.1 \%$ in women was observed compared with the specificity when BM-O was applied within employees with depressive complaints.

Analyses were performed for 12 months of follow-up as well, and similar results were found. Although slightly higher RRs were found for 12 months of follow-up, the RRs for men when the BM-O and BM-L were applied within employees with depressive complaints, failed to reach statistical significance (data not shown).

\section{Discussion}

\section{Main findings and interpretation of outcomes}

Previous research by Kant et al. found that the screening questionnaire Balansmeter proved successful in identifying employees at high risk of future sickness absence. ${ }^{13}$ The aim of the present study was to examine if the Balansmeter is able to identify employees at high risk of future sickness absence within a more specific target population, that is, employees with depressive complaints. The results of this study proved that the BM is able to predict future long-term sickness absence among employees with depressive complaints. 
In the first part of the study, the predictive properties of the BM were examined in the total study population, allowing for a direct comparison of the predictive properties of the BM in the present study with the results found in the study of the external validation of the BM. The present study was conducted at the same company in which the external validation study of the BM was carried out in 2003. ${ }^{13}$ However, sickness absence prevalence rates decreased in the Netherlands, from $4.8 \%$ in 2003 to $4.3 \%$ in 2009 . This may have influenced the predictive properties of the screening instrument in a negative way. The results of the present study showed, for both 12 and 18 months of follow-up, slightly lower but nevertheless similar RRs as the study conducted by Kant et al. ${ }^{13}$ in which a RR of 3.90 (2.35-6.45) was found in men and of 2.62 (1.44-4.77) was found in women. The sensitivity of the BM was higher in the present study (26.8\% in men and $9.4 \%$ in women) compared with the study from Kant et al. (14.3\% in men and $6.1 \%$ in women) and the specificity was slightly lower, but remained > $90 \%$. Similar to the results of Kant et al, the predictive properties of the BM-O were higher among men than women and relatively more men than women were identified at high risk of sickness absence. This is in line with the development of the $\mathrm{BM}$, in which the predictive properties proved to be better for men than for women. Although the prevalence of sickness absence and the number of long-term sickness absence spells slightly differed between the present study and the study by Kant et al., it was concluded that the predictive properties and the sensitivity and specificity remained intact.

Depressive complaints have been found to be associated with long-term sickness absence. ${ }^{16,17}$ Therefore, in the second part of the study, the predictive properties of having depressive complaints compared with not having depressive complaints were examined. The RRs of long-term sickness absence for employees with depressive complaints (HAD-D $\geq 8$ versus $H A D<8$ ) were quite high, a RR of 3.13 (2.41-4.09) was found for men and 2.45 (2.00-3.00) for women. These results confirm the results of our earlier study that was performed in a different study population. ${ }^{17}$

In the third part of the study, it was examined if the prediction of long-term sickness absence would improve when the BM was applied within employees with depressive complaints. The RR for long-term sickness absence increased to $5.23(3.13 \times 1.67)$ in men and to $3.87(2.45 \times 1.58)$ in women. These findings demonstrated that when the BM-O is applied among employees with depressive complaints, the RR of future long-term sickness absence is higher than the RR of the BM-O when applied in the total (general) study population. However, the RRs of the BM-O when applied in employees with depressive complaints were lower than the RRs of the BM-O applied in the total population. This may be explained by the fact that depressive complaints are included in the prediction of the risk of future sickness absence by the BM. The HAD-D proved to have good predictive properties itself. Nevertheless, the BM had a substantial surplus value in the prediction of long-term sickness absence.

For 12 months of follow-up, the RRs were slightly higher than the RRs for 18 months of follow-up. However, in male employees with depressive complaints, RRs were slightly lower and not statistically significant. The BM was originally developed to predict long-term sickness absence for 12 months of follow-up, however, the results of this study suggest that a timeframe of 18 months may be more appropriate to study long-term sickness absence spells. 


\section{Methodological considerations}

The good predictive properties of the BM and HAD-D found in this study can be partly ascribed to the strengths of the study. The study was conducted within one company, but the study population represented a large, heterogeneous population of male and female office workers. The risk of future sickness absence was assessed with the validated screening instrument Balansmeter. ${ }^{13}$ Depressive complaints were assessed by HAD-D, which has been found to be an adequate instrument for identification of depressive complaints among employees. ${ }^{15}$ Furthermore, outcome assessment was of high quality, since objective sickness absence data were used with one hundred percent coverage. The findings indicated that a follow-up period of at least 18 months was required to identify statistically significant differences in the prediction of future sickness absence among men. In women, RRs were statistically significant for both 12 and 18 months of follow-up. Furthermore, the prediction of sickness absence was performed for sickness absence spells $>28$ days, which are rather long sickness absence spells. Most employees who went on sick leave had relatively short sickness absence durations ranging from 1 to 28 days. Therefore, the prediction of total sickness absence duration would probably have been even better.

In this study data from a RCT were used. The intervention group of the RCT had to be taken into account when performing the analyses, since the intervention was expected to have resulted in decreased long-term sickness absence. Therefore, recalculations were performed to minimize the influence of the intervention group on the results. The proportion of long-term sickness absence of employees who participated in the intervention group was replaced by the proportion of sickness absence of the control group, since the intervention and control group were assumed to be equal, due to the random allocation of employees to the groups. Despite the random allocation, the two groups may have been different. However, these differences would then also have been a random effect. This procedure was believed to be the most optimal way to minimize the influence of the intervention group on the results of the present study.

With respect to the employees who were selected for participation in the RCT by the BM-L and the HAD-D, a considerable number of employees who scored above one of the cut-off points only, demonstrated quite high scores on BM-L or on HAD-D. From an ethical perspective, it was not permitted to leave these employees alone, since effective treatments for these individual conditions are available. Therefore, these employees were offered consultation with an occupational physician. Only $2 \%$ of these employees received some kind of treatment, which was not likely to have reduced the contrast between cases and non-cases. However, if treatment would have influenced sickness absence, it would have reduced the contrast between the groups and would have led to an underestimation of the effects found.

The original cut-off point on the BM (BM-O) identified $6.66 \%$ employees of the total study population at high risk of future sickness absence. For BM-L, $13.33 \%$ employees of the population on which the BM was developed were identified at high risk. In the present study, 3.9\% of the female employees were selected as a case and $10.1 \%$ of the male employees. These findings indicate that in the present study population fewer female and more male employees were identified at high risk of future sickness absence. 
The BM was originally developed for employees working in an office work environment. It is believed that the screening instrument may be suitable for other companies with an office work environment, because of comparable risk factors and/or (health) complaints. The study may not be representative for companies without an office work environment, since the risk of sickness absence is highly dependent of the context. Recently, new modules of the BM have been developed for employees working in industry or in health care.

\section{Practical implications}

The aim of the preventive strategy, as described in the introduction, is to identify employees at high risk of sickness absence by a screening instrument and to offer these employees a preventive intervention. In the development of the preventive strategy a single screening step including a test with high specificity was preferred, to avoid wrongfully labeling of employees as being unhealthy and to avoid unnecessary costs when treating wrongly classified employees. The preventive strategy was directed at those employees who would benefit the most.

Screening always implies a certain amount of misclassification. Due to the choice of a high specificity, the sensitivity of the screening instrument was rather low. Therefore, the effect of using a cut-off point on the BM with higher sensitivity (BM-L) was examined on the prediction of long-term sickness absence. When the BM-L was applied in employees with depressive complaints, the number of cases increased, the RRs were similar with the RRs of the BM-O, but the specificity decreased from $75.9 \%$ to $64.9 \%$ in men and from $87.3 \%$ to $78.2 \%$ in women. The use of the lower cut-off point resulted in more employees selected at high risk of sickness absence by the BM, but who will eventually not go on sick leave. The consequences of misclassification should always be considered when using cut-off points on a screening instrument. In our study, treatment of employees who will not go on sick leave will not have harmful effects, since treatment will most likely exist of some kind of psychological treatment, aimed at employees with relatively mild (health) complaints. However, there is a risk of labeling, but again this concerns relatively mild (health) complaints. In contrast, not treating employees who actually are at high risk of sickness absence and who have depressive complaints is not expected to have adverse consequences as well, since as mentioned before, employees with relatively mild complaints were selected. Besides, adequate care from the occupational health services is available. In case of sickness absence employees receive socio-medical counseling according to the nationwide guidelines. In case employees ask for help they receive consultation with an occupational physician. For our study, no serious consequences are to be expected from using a lower cut-off point on the BM. For application of the BM in different companies and settings, the use of alternative cut-off points should be considered while taking into account the consequences of misclassification.

\section{Implication for future research}

The Balansmeter proved to be able to adequately identify employees at high risk of future sickness absence within employees with depressive complaints. The availability of an effective screening instrument fulfils an important prerequisite for the use of a preventive strategy. Future research 
should examine if specific interventions for this specific target population are effective in the prevention/reduction of long-term sickness absence.

\section{Acknowledgements}

This study was financially supported by the Netherlands Organization for Health Research and Development, grant no. 62200024, by CAPHRI School for Public Health and Primary Care, Maastricht, the Netherlands and by the Occupational Health Services Beter (trade name of ABN AMRO Arbo Services B.V.) Amsterdam, the Netherlands. 


\section{References}

1. Marmot M, Feeney A, Shipley M, North F, Syme SL. Sickness absence as a measure of health status and functioning: from the UK Whitehall II study. J Epidemiol Community Health. 1995; 49:124-30.

2. Harvey SB, Henderson M, Lelliott $P$, Hotopf $M$. Mental health and employment: much work still to be done. Br J Psychiatry. 2009; 194:201-3.

3. Shiels C, Gabbay MB, Ford FM. Patient factors associated with duration of certified sickness absence and transition to long-term incapacity. Br J Gen Pract. 2004; 54:86-91.

4. Bultmann $U$, Rugulies R, Lund T, Christensen KB, Labriola M, Burr H. Depressive symptoms and the risk of long-term sickness absence: a prospective study among 4747 employees in Denmark. Soc Psychiatry Psychiatr Epidemiol. 2006; 41:875-80.

5. Koopmans PC, Roelen CA, Groothoff JW. Sickness absence due to depressive symptoms. Int Arch Occup Environ Health. 2008; 81:711-9.

6. Roelen CA, Koopmans PC, Hoedeman R, Bultmann U, Groothoff JW, van der Klink JJ. Trends in the incidence of sickness absence due to common mental disorders between 2001 and 2007 in the Netherlands. Eur J Public Health. 2009; 19:625-30.

7. Sanderson K, Andrews G. Common mental disorders in the workforce: recent findings from descriptive and social epidemiology. Can J Psychiatry. 2006; 51:63-75.

8. Koppes L, Klein Hesselink J, Mol M, van den Bossche S. De Nationale enquête arbeidsomstandigheden 2008 [National Survey Working Conditions 2008]. Hoofddorp: TNO Kwaliteit van Leven [TNO Quality of Life]; 2009.

9. Brouwers EP, Tiemens BG, Terluin B, Verhaak PF. Effectiveness of an intervention to reduce sickness absence in patients with emotional distress or minor mental disorders: a randomized controlled effectiveness trial. Gen Hosp Psychiatry. 2006; 28:223-9.

10. Huibers MJ, Beurskens AJ, Van Schayck CP, Bazelmans E, Metsemakers JF, Knottnerus JA, et al. Efficacy of cognitive-behavioural therapy by general practitioners for unexplained fatigue among employees: Randomised controlled trial. Br J Psychiatry. 2004; 184:240-6.

11. Nystuen $P$, Hagen KB. Solution-focused intervention for sick listed employees with psychological problems or muscle skeletal pain: a randomised controlled trial. BMC Public Health. 2006; 6:69.

12. Sogaard HJ, Bech P. The effect on length of sickness absence by recognition of undetected psychiatric disorder in long-term sickness absence. A randomized controlled trial. Scand J Public Health. 2009; 37:864-71.

13. Kant IJ, Jansen NW, van Amelsvoort LG, Swaen GM, van Leusden R, Berkouwer A. Screening questionnaire Balansmeter proved successful in predicting future long-term sickness absence in office workers. J Clin Epidemiol. 2009; 62:408-414.

14. Kant I, Jansen NW, van Amelsvoort LG, van Leusden R, Berkouwer A. Structured early consultation with the occupational physician reduces sickness absence among office workers at high risk for long-term sickness absence: a randomized controlled trial. J Occup Rehabil. 2008; 18:79-86.

15. Andrea H, Bultmann U, Beurskens AJ, Swaen GM, van Schayck CP, Kant IJ. Anxiety and depression in the working population using the HAD Scale--psychometrics, prevalence and relationships with psychosocial 
work characteristics. Soc Psychiatry Psychiatr Epidemiol. 2004; 39:637-46.

16. Hjarsbech PU, Andersen RV, Christensen KB, Aust B, Borg V, Rugulies R. Clinical and non-clinical depressive symptoms and risk of long-term sickness absence among female employees in the Danish eldercare sector. J Affect Disord. Published Online First: 24 August 2010. doi:10.1016/j.jad.2010.07.033

17. Lexis MA, Jansen NW, van Amelsvoort LG, van den Brandt PA, Kant I. Depressive complaints as a predictor of sickness absence among the working population. J Occup Environ Med. 2009; 51:887-95.

18. Lexis MAS, Jansen NWH, Huibers MJH, Van Amelsvoort LGPM, Berkouwer A, Tjin A Ton G, et al. Prevention of long-term sickness absence and major depression in high-risk employees: a randomised controlled trial. Occup Environ Med. Published Online October 5, 2010. doi: 10.1136/oem.2010.057877.

19. Snaith RP. The Hospital Anxiety and Depression Scale. Health Qual Life Outcomes. 2003; 1:29.

20. Zigmond AS, Snaith RP. The Hospital Anxiety and Depression Scale. Acta psychiatr Scand. 1983; 67:361-70.

21. Bjelland I, Dahl AA, Haug TT, Neckelmann D. The validity of the Hospital Anxiety and Depression Scale. An updated literature review. J Psychosom Res. 2002; 52:69-77.

22. Hensing G, Alexanderson K, Allebeck P, Bjurulf P. Sick-leave due to psychiatric disorder: higher incidence among women and longer duration for men. Br J Psychiatry. 1996; 169:740-6.

23. Laaksonen M, Martikainen P, Rahkonen O, Lahelma E. Explanations for gender differences in sickness absence: evidence from middle-aged municipal employees from Finland. Occup Environ Med. 2008; 65:32530.

24. Smeby L, Bruusgaard D, Claussen B. Sickness absence: could gender divide be explained by occupation, income, mental distress and health? Scand J Public Health. 2009; 37:674-81.

25. Van de Velde S, Bracke P, Levecque K. Gender differences in depression in 23 European countries. Crossnational variation in the gender gap in depression. Soc Sci Med. 1982; 71:305-13. 



\section{4}

\section{Experience of health complaints and help seeking behavior in employees screened for depressive complaints and risk of future sickness absence}

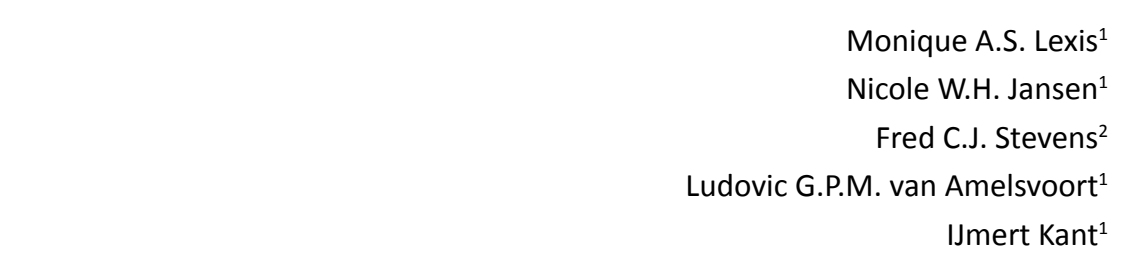

${ }^{1}$ CAPHRI School for Public Health and Primary Care, Department of Epidemiology, Maastricht University, Maastricht, The Netherlands ${ }^{2}$ Department of Educational Development and Research, Maastricht University, Maastricht, The Netherlands 


\section{Abstract}

\section{Introduction}

The aim of this study was to examine the associations between on the one hand depressive complaints and risk of future sickness absence and on the other hand experience of health complaints and help seeking behavior in the working population.

\section{Methods}

Cross-sectional data were used from employees working in the banking sector $(n=8,498)$. The screening instrument included measures to examine the risk of future sickness absence, depressive complaints and help seeking behavior.

\section{Results}

Of employees reporting health complaints, approximately $80 \%$ had already sought help for these complaints. Experience of health complaints and subsequent help seeking behavior differed between employees with mild to severe depressive complaints and employees at risk of future sickness absence. Experience of health complaints was highest in employees identified with both concepts (69\%) compared with employees identified at risk of future sickness absence only $(48 \%)$ and with mild to severe depressive complaints only (57\%). In those employees identified with one or both concepts and who had not sought help already, the intention to seek help was about $50 \%$.

\section{Conclusions}

From a screening perspective, employees who do not experience health complaints or who do not have the intention to seek help may refuse participation in early intervention. This might be a bottleneck in the implementation of preventive interventions in the occupational health setting. 


\section{Introduction}

In occupational health settings, interventions aimed at sickness absence of employees have increased during the past 20 years. ${ }^{1}$ Most of the interventions focus on reintegration to work when sickness absence has already occurred..$^{2-4}$ Many studies have shown that treatment and reintegration have limited effects and that work resumption remains difficult. ${ }^{5-8}$ Recently, a preventive strategy was developed in which employees at risk of future sickness absence receive early intervention to prevent future sickness absence. ${ }^{9}$ Previous research by Kant et al. demonstrated that it is possible to identify employees at high risk of future sickness absence. ${ }^{10}$ In an additional study it was demonstrated that preventive intervention among this group proved successful in reducing future sickness absence. ${ }^{9}$ However, the latter study also revealed that the willingness and motivation of employees to participate in the preventive intervention varied substantially between subjects. Thus, having disposal of an adequate screening instrument as well as having a successful intervention does not necessarily result in successful prevention of sickness absence. An important reason for not participating in the preventive intervention could have been that employees, identified by the screening instrument at high risk of future sickness absence, did not experience or recognize any physical or mental health complaints at the time of the screening. ${ }^{9}$ Consequently, these employees were not likely to display any help seeking behavior. This finding may imply a point of concern when conducting preventive interventions. Besides experience of health complaints, help seeking behavior may be influenced by many other factors as well, for example gender, age and lay referral. ${ }^{11-13}$ According to the Health Belief Model (HBM), ${ }^{14,15}$ experience of health complaints is the most important determinant of help seeking behavior. Especially subjective experience of health complaints, defined as 'illness', determines help seeking behavior. ${ }^{16-20}$ The HBM furthermore states that help seeking depends strongly on the individuals' perception that being ill may have serious consequences in terms of disease. Although the general principles of help seeking behavior apply to workers as well, the process may be different when compared with the general population. Workrelated factors, such as work conditions and work environment, have been mentioned as important factors that can contribute to development of health complaints and induce help seeking behavior. Interference of illness with work and disruption of the working situation may be an important reason to seek help in employees. ${ }^{20-22}$ Employees might be expected to be aware of health complaints more promptly and seek help in a relatively early stage compared with the general population, since illness may lead to sickness absence and disability and it may become a threat to a person's income.

Recently, a randomized controlled trial (RCT) has been conducted to examine the efficacy of a preventive intervention in employees identified at high risk of sickness absence and with mild depressive complaints on the prevention of long-term sickness absence and major depression. The preventive intervention was specifically targeted at employees with depressive complaints, since depressive complaints were found to be highly prevalent in the working population and strongly associated with sickness absence. ${ }^{23}$ Data of the RCT offered us the possibility to study the experience of health complaints and help seeking behavior among employees for two different concepts, that 
is, depressive complaints and the risk of future sickness absence. In case of screening for depressive complaints, the questionnaire assesses a person's experience of depressive symptoms. It may be expected that employees who experience depressive (=health) complaints at the time of completing the screening questionnaire will actually recognize these complaints and report them. In contrast with this, the concept of risk of sickness absence assesses a person's vulnerability for an event to happen in the future. This concept assesses a combination of factors that together determine the risk of future sickness absence. This multitude of factors does not necessarily have to be related to the experience of health complaints. It may occur that the increased risk can be mainly explained by work factors, such as having conflicts at work. In this situation it is likely that employees do not recognize health complaints at the moment of screening and do not recognize their 'being at risk' for a future event. The aim of this study is to examine the experience of health complaints and help seeking behavior among the working population in relation to: 1) depressive complaints, 2) risk of sickness absence and 3 ) the combination of both concepts. It was hypothesized that employees identified with mild to severe depressive complaints will report to experience more health complaints compared with employees identified at high risk of future sickness absence. Moreover, it was hypothesized that in employees who do experience health complaints and who have not sought help already, those employees meeting the criteria for both concepts will have the highest intention to seek help. Additionally, specific characteristics of the employees were studied to investigate whether sub groups can be distinguished which might need a different guidance towards preventive intervention.

\section{Methods}

\section{Design and study population}

This cross-sectional study examined the relationship between on the one hand depressive complaints and risk of future sickness absence and on the other hand experience of health complaints and help seeking behavior. The study was performed among employees working at a large banking company in the Netherlands and is part of a randomized controlled trial (RCT) that aims to investigate the efficacy of early intervention on the prevention of future sickness absence and major depression. For the current study, data from the screening questionnaires of the RCT were used. In 2007, 23,973 employees of the company received a screening questionnaire at their home address. The screening instrument included questionnaires to assess the risk of sickness absence, depressive complaints and included questions to examine the experience of health complaints and help seeking behavior. In the invitation letter, employees were asked to provide written informed consent. The informed consent covered the usage of the questionnaire data of the screening and the possibility to participate in the trial. After two weeks a reminder was sent to all non-respondents. Four separate batches were necessary to realize smooth processing of the large amount of returned questionnaires and to guarantee smooth enrolment of participants in the trial. Screening questionnaires were sent out in February $(n=7,000)$, $\operatorname{March}(n=4,500)$, September $(n=6,196)$ and October $(n=6,277) 2007$. In total, 9,157 employees (38.2\%) 
responded to the screening instrument. 8,892 questionnaires were suitable for the analyses, 265 were discarded due to technical reasons, such as unreadable questionnaires or missing informed consent.

\section{Measurements}

The screening questionnaire contained questions to assess self-reported information on the exposure variables 1) risk of future sickness absence, 2) depressive complaints and to assess the outcome variables 3 ) experience of health complaints and 4) help seeking behavior.

\section{Risk of future sickness absence}

The Balansmeter (BM), which has been specifically developed for employees working in an office environment, was used to identify employees at high risk of future sickness absence. The BM was developed and validated in the Maastricht Cohort Study ${ }^{10,24}$ and includes 19 individual predictors for men and 22 for women. Due to overlapping predictors for men and women, the BM includes 34 multiple choice questions regarding demographics, work environment, private situation, (mental) health status, help seeking behavior, and sickness absence history. Using an algorithm based on the weighted factors of the individual items of the model, a total score can be calculated, with higher scores indicating a higher risk of future long-term sickness absence. In this study, in women a cutoff point with $87.9 \%$ specificity and $52.6 \%$ sensitivity was applied (score on BM -1.069 ) and in men specificity was $87.8 \%$ and sensitivity was $65.1 \%$ (score on BM -1.944 ).

\section{Depressive complaints}

The Hospital Anxiety and Depression (HAD) scale was used to assess the presence and severity of depressive complaints by self-report. The HAD is a 14 item self-report questionnaire, that was originally developed to indicate the presence and severity of both anxiety (HAD-A) and depression (HAD-D) separately. ${ }^{25,}{ }^{26}$ Both the HAD-A and the HAD-D consist of 7 items and are scored on a four point Likert scale (0 to 3), resulting in a range of 0 to 21 . In this study only the HAD-D was used. Although the HAD scale was originally developed to identify caseness of anxiety disorders and depression among patients in non-psychiatric hospital clinics, the questionnaire was recently found to perform well in assessing the symptom severity and caseness of anxiety disorders and depression in somatic, psychiatric and primary care patients and in the general population ${ }^{27}$ and also in the working population. ${ }^{28}$ With respect to the cut-off values, we used the values suggested by Zigmond and Snaith. ${ }^{26} \mathrm{~A}$ higher score on the HAD-D indicates more severe depressive complaints. Employees were defined as having mild to severe depressive complaints when they scored 8 points or higher on the HAD-D. Less than 8 points was defined as scoring in the normal range of the scale.

\section{Experience of health complaints and help seeking behavior}

The screening instrument contained four questions on the outcomes experience of health complaints and help seeking behavior. The first question was: 'Do you currently experience any health complaints or health problems?' (yes/no). When the answer to this question was 'yes', the second question 
was: 'Have you already sought help for these complaints or problems?' (yes/no), and if the answer was yes, the third question was 'by whom?' (general practitioner and/or occupational physician and/ or someone else). The fourth question investigated whether employees, who did not visit someone for their health complaints, have the intention to seek help in the near future (yes/no).

\section{Demographic, health-related and work-related factors}

Employees provided information on gender, age, educational level, presence of a long-term illness (having the disease for more than six months), current sickness absence from work, experience of a life event during the past year, job insecurity and having an executive function through self-report in the questionnaire. Co-worker social support and skill competence were measured with a validated Dutch version of the self-administered Job Content Questionnaire (JCQ). ${ }^{29,} 30$

\section{In- and exclusion criteria of the total study population}

Employees who responded to the screening questionnaire and who gave informed consent were included in the study. Those employees who were completely or partially on sick leave at the time of completing the screening questionnaire and female employees who were pregnant or on pregnancy leave were excluded from the analyses to avoid measuring help seeking behavior due to pregnancyrelated sickness absence and pregnancy-related health complaints.

\section{Definition of three study populations}

Three populations were selected to study the concept of risk of sickness absence, the concept of depressive complaints and the concept of both risk of sickness absence and depressive complaints. For each population cases and non-cases were defined. The first study population was based on risk of future sickness absence (group A) and consisted of employees who scored above or below the predefined cut-off points (for women -1.069, for men -1.944) of the BM, indicating 1,001 cases and 6,509 non-cases. A score above the cut-off point indicates that the employee is at high risk of future sickness absence. The second study population was based on depressive complaints (group B). A score above the cut-off point of 8 points on HAD-D indicated mild to severe depressive complaints (cases, $n=653$ ), a score below 8 indicated no to mild depressive complaints (non-cases, $n=7,224$ ). The third population was based on scores on both concepts (group C) and consisted of those employees who scored above (cases, $n=204$ ) both cut-off points of the BM and HAD-D and, in contrast, of those employees who scored below (non-cases, $n=7,202$ ) one or both cut-off points.

\section{Statistical analysis}

First, independent samples t-tests and Chi-square tests were used to examine differences in experience of health complaints and intention to seek help in employees who responded to the screening instrument. These data were described for factors related to the experience of health complaints and help seeking behavior, such as demographic, health-related, private situation-related and work-related factors. 
Second, cross tabulations were calculated to describe differences in the experience of health complaints in employees identified 1) at risk versus not at risk of future sickness absence (group A), 2) with versus without mild to severe depressive complaints (group B) and 3) at risk of future sickness absence and with mild to severe depressive complaints versus employees not at risk of sickness absence and/or without mild to severe depressive complaints (group C).

Additionally, Chi-square tests were used to test differences in the experience of health complaints and help seeking behavior for several sub groups. Results were displayed for four potentially relevant sub groups, that is, severity of the complaints, gender, presence of long-term illness and educational level. Statistical analyses were performed using SPSS 15.0.

\section{Results}

Figure 1 Experience of health complaints and help seeking behavior

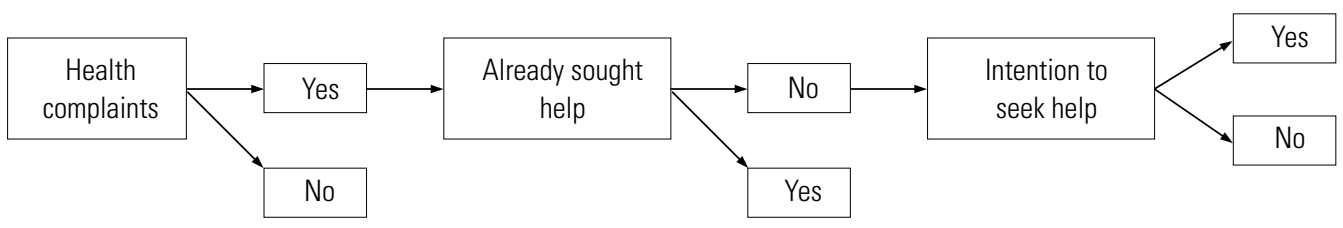

\section{Results for all respondents on the screening questionnaire}

Figure 1 displays the three main questions on help seeking behavior included in the screening instrument. Table 1 presents the experience of health complaints and help seeking behavior among employees responding to the screening instrument. Approximately $2,311(27 \%)$ respondents reported to experience health complaints. Of these, 1,848 (80\%) employees already sought help for their health complaints. 1,293 employees sought help at a general practitioner, 341 at an occupational physician and 951 employees received help from someone else. Of the 463 employees who have not sought help already, $35 \%$ had the intention to seek help for the health complaints, whereas $46 \%$ had no intention. Regarding the descriptive characteristics of the study population, marked and statistically significant differences were found between the groups 'experience of health complaints no' (Table 1) and 'experience of health complaints yes and already sought help yes' with respect to demographics and factors regarding the private and work situation, except for skill discretion (= skill competence). Table 1 also presents the intention to seek help in employees who reported to experience health complaints but who had not sought help already. Most of the characteristics were comparable between those who reported to have and those who reported not to have the intention to seek help. However, in employees who had no intention to seek help, absence from work was significantly higher and more employees were having an executive position compared with those employees who did have the intention to seek help. 
Table 1 Experience of health complaints and help seeking behavior in respondents on the screening instrument

\begin{tabular}{|c|c|c|c|c|c|c|}
\hline & $\begin{array}{l}\text { Health } \\
\text { complaints } \\
\text { NO } \\
(n=6,187)^{*}\end{array}$ & $\begin{array}{l}\text { Health } \\
\text { complaints } \\
\text { YES ( } n=2,311) \\
\text { already } \\
\text { sought help } \\
\text { YES } \\
(n=1,848)^{*}\end{array}$ & $p$-value & $\begin{array}{l}\text { Health } \\
\text { complaints } \\
\text { YES ( } n=2,311) \\
\text { Already } \\
\text { sought help } \\
\text { NO ( } n=463) \\
\text { Intention to } \\
\text { seek help YES } \\
\text { (n=164) } †\end{array}$ & $\begin{array}{l}\text { Health } \\
\text { complaints } \\
\text { YES ( } n=2,311) \\
\text { Already } \\
\text { sought help } \\
\text { NO ( } n=463) \\
\text { Intention to } \\
\text { seek help NO } \\
(n=213) \dagger\end{array}$ & $\mathrm{p}$-value \\
\hline \multicolumn{7}{|l|}{ Demographics } \\
\hline Male; $(\%)$ & 52.1 & 44.9 & $<0.001$ & 50.0 & 49.8 & 0.964 \\
\hline Age; mean (SD) & 42.08 (9.75) & 45.00 (9.24) & $<0.001$ & $42.31(9.72)$ & $43.34(11.10)$ & 0.165 \\
\hline Educational level & & & $<0.001$ & & & 0.782 \\
\hline Low, Medium; (\%) & 56.4 & 70.0 & & 61.9 & 63.4 & \\
\hline High; (\%) & 43.6 & 30.0 & & 38.1 & 36.6 & \\
\hline \multicolumn{7}{|l|}{ Health } \\
\hline $\begin{array}{l}\text { Long-term illness; } \\
\text { (\% yes) }\end{array}$ & 15.0 & 62.6 & $<0.001$ & 36.2 & 37.7 & 0.760 \\
\hline $\begin{array}{l}\text { Absent from work; } \\
\text { (\% yes) }\end{array}$ & 1.2 & 19.5 & $<0.001$ & 2.5 & 7.5 & 0.030 \\
\hline \multicolumn{7}{|l|}{ Private situation } \\
\hline $\begin{array}{l}\text { Experience of life- } \\
\text { event; ( } \% \text { yes) }\end{array}$ & 32.3 & 44.2 & $<0.001$ & 40.5 & 40.6 & 0.988 \\
\hline \multicolumn{7}{|l|}{$\begin{array}{l}\text { Work } \\
\text { characteristics }\end{array}$} \\
\hline $\begin{array}{l}\text { Coworker social } \\
\text { support (4-16) ‡; } \\
\text { mean (SD) }\end{array}$ & $12.66(1.57)$ & $12.37(1.75)$ & $<0.001$ & $12.36(1.66)$ & $12.23(1.60)$ & 0.342 \\
\hline $\begin{array}{l}\text { Skill discretion } \\
\text { (12-48) ‡; mean (SD) }\end{array}$ & $37.16(5.13)$ & $36.03(5.51)$ & 0.152 & $36.65(5.56)$ & $36.05(5.41)$ & 0.669 \\
\hline $\begin{array}{l}\text { Job insecurity; } \\
\text { (\% yes) }\end{array}$ & 12.5 & 23.4 & $<0.001$ & 16.0 & 23.0 & 0.098 \\
\hline $\begin{array}{l}\text { Executive position; } \\
\text { (\% yes) }\end{array}$ & 21.6 & 15.8 & $<0.001$ & 23.2 & 14.6 & 0.032 \\
\hline
\end{tabular}

* Total of cases does not equal 8,892 due to missing values; † Total of cases does not equal 463 due to missing values; $\ddagger$ scale range. 
The association between risk of sickness absence, depressive complaints, both concepts and experience of health complaints

Of all employees responding to the screening instrument, $13.3 \%$ was identified at high risk of future sickness absence and $8.3 \%$ was identified with mild to severe depressive complaints. Of the employees identified at high risk of sickness absence, $48 \%$ reported to experience health complaints, compared with $20 \%$ of the employees identified not at risk (group A) (Table 2). In contrast, the experience of health complaints in employees identified with depressive complaints was higher (57\%), in comparison with $21 \%$ of the employees without depressive complaints (group B). When looking at employees meeting the criteria for both concepts (group C), 69\% reported to experience health complaints. We additionally studied the effect of cut-off points based on a higher specificity for both risk of sickness absence (94.4\% for men and $94.7 \%$ for women) and depressive complaints (HAD-D $\geq 11$ ) on the experience of health complaints, since the choice of a cut-off point is relatively arbitrary and therefore may have influenced the results (specific data not shown). When applying these cut-off points, experience of health complaints for risk of future sickness absence increased to $58 \%$, for depressive complaints to $68 \%$ and for both concepts together to $75 \%$.

Table 2 Association between the risk of future sickness absence (SA) (group A), depressive complaints (group B), both risk of future $S A \&$ depressive complaints (group C) and experience of health complaints

\begin{tabular}{|c|c|c|c|c|}
\hline & $\begin{array}{l}\text { Experience of } \\
\text { health complaints } \\
\text { yes }\end{array}$ & $\begin{array}{l}\text { Experience of } \\
\text { health complaints } \\
\text { no }\end{array}$ & Total & p-value \\
\hline At high risk of future $S A$ & $484(48 \%)$ & $517(52 \%)$ & 1,001 & $<0.001$ \\
\hline Not at risk of future SA & $1,293(20 \%)$ & $5,216(80 \%)$ & 6,509 & \\
\hline Total * & 1,777 & 5,733 & 7,510 & \\
\hline Mild to severe depressive complaints & $370(57 \%)$ & $283(43 \%)$ & 653 & $<0.001$ \\
\hline No to mild depressive complaints & $1,508(21 \%)$ & $5,716(79 \%)$ & 7,224 & \\
\hline Total * & 1,878 & 5,999 & 7,877 & \\
\hline $\begin{array}{l}\text { At risk of future SA and mild to severe } \\
\text { depressive complaints }\end{array}$ & $140(69 \%)$ & $64(31 \%)$ & 204 & $<0.001$ \\
\hline $\begin{array}{l}\text { (1 - At risk of future SA and / or mild to } \\
\text { severe depressive complaints) }\end{array}$ & $1,609(22 \%)$ & $5,593(78 \%)$ & 7,202 & \\
\hline Total * & 1,749 & 5,657 & 7,406 & \\
\hline
\end{tabular}

* Totals of cases do not equal 8,892 due to missing values. 
Table 3 Help seeking behavior in employees identified at risk of future sickness absence (SA), with mild to severe depressive complaints and both at risk of future SA \& with mild to severe depressive complaints

\begin{tabular}{|c|c|c|c|c|c|c|c|c|c|}
\hline & \multicolumn{3}{|c|}{$\begin{array}{l}\text { Experience of health } \\
\text { complaints }\end{array}$} & \multicolumn{3}{|c|}{ Already sought help } & \multicolumn{3}{|c|}{ Intention to seek help } \\
\hline & No & Yes & $\mathrm{p}$-value & Yes & No & $\mathrm{p}$-value & Yes & No & $\mathrm{p}$-value \\
\hline Risk of $S A(B M \geq 13,3)$ & 517 & 484 & $<0.001$ & 404 & 80 & $<0.001$ & 31 & 37 & 0.875 \\
\hline Severity $(\mathrm{BM} \geq 6,6) ;(\%$ yes) & 42.0 & 62.6 & $<0.001$ & 63.1 & 60.0 & 0.598 & 61.3 & 56.8 & 0.705 \\
\hline Male; (\% yes) & 73.9 & 66.1 & 0.007 & 67.1 & 61.3 & 0.314 & 58.1 & 62.2 & 0.731 \\
\hline Long-term illness; ( $\%$ yes) & 29.0 & 66.8 & $<0.001$ & 70.0 & 51.3 & 0.001 & 38.7 & 55.6 & 0.169 \\
\hline Educational level high; (\% yes) & 19.0 & 19.0 & 0.986 & 18.2 & 23.1 & 0.313 & 26.7 & 19.4 & 0.486 \\
\hline $\begin{array}{l}\text { Depressive complaints } \\
\text { (HAD-D } \geq 8)\end{array}$ & 283 & 370 & $<0.001$ & 259 & 111 & $<0.001$ & 41 & 53 & 0.782 \\
\hline Severity (HAD $\geq 11) ;(\%$ yes) & 21.2 & 34.3 & $<0.001$ & 35.9 & 30.6 & 0.327 & 29.3 & 28.3 & 0.918 \\
\hline Male; (\% yes) & 51.2 & 53.0 & 0.660 & 50.6 & 58.6 & 0.159 & 53.7 & 58.5 & 0.639 \\
\hline Long-term illness; (\% yes) & 19.4 & 54.9 & $<0.001$ & 64.7 & 44.3 & $<0.001$ & 46.3 & 38.0 & 0.422 \\
\hline Educational level high; (\% yes) & 35.4 & 26.9 & 0.024 & 25.7 & 29.5 & 0.461 & 40.0 & 24.5 & 0.117 \\
\hline $\begin{array}{l}\text { At risk of SA \& depressive } \\
\text { complaints } \\
(B M \geq 13,3 \text { and } H A D-D \geq 8)\end{array}$ & 64 & 140 & $<0.001$ & 105 & 35 & 0.484 & 16 & 14 & 0.321 \\
\hline $\begin{array}{l}\text { Severity (BM } \geq 6,6 \text { and } \\
\text { HAD-D } \geq 8) ;(\% \text { yes) }\end{array}$ & 53.1 & 72.9 & 0.006 & 75.2 & 65.7 & 0.273 & 62.5 & 64.3 & 0.919 \\
\hline Male; (\% yes) & 57.8 & 63.6 & 0.432 & 64.8 & 60.0 & 0.612 & 50.0 & 57.1 & 0.696 \\
\hline Long-term illness; ( $\%$ yes) & 25.4 & 63.8 & $<0.001$ & 67.0 & 54.5 & 0.198 & 56.3 & 46.2 & 0.588 \\
\hline Educational level high; (\% yes) & 21.0 & 16.2 & 0.414 & 16.5 & 15.2 & 0.856 & 26.7 & 7.7 & 0.191 \\
\hline
\end{tabular}

\section{Characteristics of the three study populations}

Table 3 presents help seeking behavior in employees selected by the screening instrument as eligible candidates for participation in preventive intervention. Eligible candidates were employees identified at risk of sickness absence (group A), with mild to severe depressive complaints (group B) or meeting the criteria of both concepts simultaneously (group C). In employees meeting the criteria of both concepts (group C), the experience of health complaints (140 / 204) and the intention to seek help in employees who have not sought help already for these health complaints (16/30) was found to be highest compared with groups $\mathrm{A}$ and $\mathrm{B}$.

Statistically significant differences were found in the experience of health complaints when looking at the severity of complaints and presence of a long-term illness in all three concepts. Significant gender differences were found only for risk of future sickness absence, with more men than women experiencing health complaints. Significant differences in educational level were found only for depressive complaints, with employees having a low or medium educational level experiencing more health complaints. When looking at 'already sought help', among employees at risk of future sickness absence and among employees with mild to severe depressive complaints, those employees having a long-term illness have sought significantly more help for their health complaints already 
compared with those without a long-term illness. Of the employees who reported to experience health complaints and who have not sought help already, the intention to seek help proved rather low, approximately $50 \%$ in all three concepts. No statistically significant differences were found for the intention to seek help with regard to the severity of complaints, gender, presence of a long-term illness and educational level.

\section{Discussion}

\section{Main findings}

To our knowledge, this the first study that aimed at quantifying help seeking behavior specifically in the working population, by studying the associations between employees identified with depressive complaints and/or at risk of future sickness absence and their experience of health complaints and subsequent help seeking behavior. From the general population it is known that only a relatively small part of the population experiencing symptoms/health complaints seeks help, called the clinical iceberg. ${ }^{31}$ In our study, approximately $80 \%$ of the employees who reported to experience health complaints had already sought help for their health complaints. This high percentage may be explained by the theory of illness behavior and the HBM, both stating that help seeking behavior is strongly affected by the possible negative consequences of health complaints. For employees, health complaints may have serious consequences for functioning at work or may even result in losing one's job, where loss of income then may become a serious threat. This may induce help seeking behavior in this population. Of the employees who already sought help, $18.5 \%$ received help from an occupational physician of the banking company. This may have been influenced by the nature of the occupational health services (OHS) of the banking company under study. The OHS have regular contacts with their employees during the year and can be characterized as being easily accessible for the employees.

Regarding the results of all respondents on the screening instrument, significant differences were found in the characteristics of employees who did and who did not experience health complaints. Experience of health complaints was significantly higher in female employees, in employees having a higher age, a lower educational level, a long-term illness, which is in line with the literature about illness behavior. ${ }^{12,32-34}$ Experience of health complaints and the intention to seek help was higher in employees having an executive position. Experience of health complaints was also higher in those employees who reported high job insecurity. This may be explained by the fact that health complaints may have more (serious) adverse consequences in case of having an executive function or having fear to lose one's job.

Of all employees responding to the screening instrument, approximately $30 \%$ indicated to experience health complaints. Regarding the results of the employees screened for risk of future sickness absence (group A), for depressive complaints (group B), and for both concepts (group C), it was expected that many employees screened 'positively' would actually report to experience health complaints. 
However, the results demonstrated that this did not turn out to be the case. When comparing the results of the three groups with the results of all respondents together, experience of health complaints increased among the employees identified at risk of future sickness absence (48\%) and also among those identified with mild to severe depressive complaints (57\%). Experience of health complaints was highest among those identified with both concepts (69\%). Especially for group B and group $\mathrm{C}$, more employees were expected to have reported health complaints. However, from those employees identified with mild to severe depressive complaints, still $43 \%$ of the respondents did not report to experience health complaints at that moment. This might be due to the relatively mild level of health complaints the screening instrument aimed at.

Employees identified with more severe complaints and employees with a long-term illness reported to experience significantly more health complaints in each of the three concepts. Employees with a long-term illness had also sought significantly more help already. Significantly more health complaints were experienced by those employees identified with depressive complaints with a low or medium educational level. These findings are in line with the literature, as mentioned earlier. However, in employees identified at risk of sickness absence men were found to experience more health complaints than women. This is in contrast with studies conducted in the general population and also in the working population, in which women usually tend to report more health complaints. ${ }^{33-36}$ As compared with the general population, differences may exist regarding experience of health complaints and help seeking behavior in the working population. First, people being in poor health are not likely to enter the labor force. Second, employees who develop (serious) bad health over time are likely to leave the labor force at a certain point in time. For example, women who have a long-term illness may not even start working or they may quit working when they get ill. This is different for men, who more often occupy the role of breadwinner and therefore need to start working and/or remain working. An earlier study observed that the vast majority of men with a small part-time job reported to have a long-term illness. ${ }^{37}$ These selection effects may also explain differences in prevalence of especially the more severe health complaints in the general and working population, since employees with more severe complaints may not enter at all or may have already left the labor force. This may result in an underestimation of severe health complaints in the working population. Consequently, a higher prevalence of people with severe health complaints is more likely to be observed in the general population.

Approximately $70 \%$ of all employees identified with both mild to severe depressive complaints and at risk of sickness absence indicated to recognize health complaints. This implies that still $30 \%$ of the employees screened positively on both concepts indicated not to experience health complaints. Regarding the intention to seek help, in all three concepts the intention to seek help was about $50 \%$. Of all employees who reported to experience health complaints and who have not already sought help for these complaints, $50 \%$ indicated to have no intention to seek help. These two findings are of special importance from the perspective of early intervention. The main assumption of this study was that experience of health complaints is a prerequisite for help seeking behavior. Additionally, experience of health complaints did not automatically imply an intention to seek help. When these employees are 
offered the possibility to participate in early intervention, no experience of health complaints or not having the intention to seek help may constitute a bottleneck in participation in early intervention.

\section{Methodological considerations}

The following issues should be kept in mind when interpreting the results of this study. We studied the association between mild to severe depressive complaints, risk of future sickness absence and the experience of health complaints. The screening questionnaire investigated help seeking behavior due to health complaints. However, we cannot state with certainty that the reported health complaints were caused by depressive complaints or by the factors included to measure risk of sickness absence, since data on the reasons for the health complaints was not available. These reasons may include factors such as conflicts at work, marital problems or financial problems. Because the design of the study is cross-sectional, conclusions about causes and effects can not be drawn from these results. Data on the intention to seek help for health complaints were assessed by the screening questionnaire. However, data on the actual participation of employees allocated to the intervention were not available. The relationship between intention to seek help and actual participation in preventive intervention could not be studied.

The cut-off point of the HAD-D was set at 8 points or higher indicating a range from mild to severe depressive complaints. Because this range is very broad, differences in the experience of health complaints within the group of depressive complaints may certainly appear. This may also apply for risk of sickness absence, since a relatively mild cut-off point was chosen. Since the choice for a cut-off point always remains arbitrary, we explored the impact of cut-off points based on a higher specificity for both concepts. Experience of health complaints increased indeed, but reached in neither case over $75 \%$. When screening for both being at risk of sickness absence and depressive complaints there is still $25 \%$ of the respondents who do not report health complaints.

When applying a screening instrument, misclassification of participants is likely to occur, resulting in false-positive or false-negative classification. In case of risk of sickness absence, a high specificity was preferred when the questionnaire was developed. For men specificity was $94.4 \%$ and in women 94.7\%. Thus, approximately $5.5 \%$ of all participants will be classified as false-positives, employees who are not at risk actually, but nevertheless classified at risk by the screening instrument. From research on the development of the HAD-scale by Zigmond and Snaith, it can be derived that the HAD-D has a very high specificity as well. ${ }^{26}$ Our results showed that $52 \%$ of the employees at risk of sickness absence and $43 \%$ of the employees with mild to severe depressive complaints do not experience health complaints. This indicates that these percentages could only be explained for a small part by misclassification by the screening instrument.

Although many important factors often mentioned in the literature to be associated with illness behavior were included in the analyses, some of them were or could not be included in our questionnaire, such as data on ethnicity and cultural background.

The majority of the employees screened 'positively' for depressive complaints, risk of future sickness absence and meeting the criteria for both concepts, did already seek help for their health complaints. 
These employees have already undertaken action themselves to solve their problems and therefore they do not belong to the target group the preventive strategy aims at.

\section{Implications for future research}

The present study gained insight in the background of help seeking behavior of employees identified as eligible candidates for participation in preventive intervention. The results found may contribute to the explanation of the relatively low participation of employees in preventive intervention. Our results demonstrated that most of the employees reporting health complaints already sought help for these complaints. Of the employees identified at risk of future sickness absence, with mild to severe depressive complaints or identified with both concepts, still 30 to $50 \%$ did report not to experience health complaints and of these approximately $50 \%$ did not have an intention to seek help. This group of employees may include those most likely to refuse participation in preventive intervention, while intervention could be beneficial in reducing the risk of future sickness absence and/or depressive complaints. Professionals working in occupational health care should take this into account in case of early intervention.

Besides the experience of health complaints, there are many other factors that may determine participation and successful implementation of preventive interventions, such as the ability to offer voluntary participation, the possibility of participation in and after working hours, a location within easy reach and for example the person's belief in the effectiveness of the intervention. Participation may also depend on many other factors as described in the Health Belief Model, such as personality, self-efficacy, cues to action and an individual's personal considerations, for example fear to lose one's job. Employees may refuse participation because they do not want their problems to become noticeable for the boss and/or colleagues. Since our study was carried out within one banking company, the influence of factors such as the corporate culture could not be investigated, but might have an important role as well. All these factors may contribute remarkably to the willingness to participate in preventive intervention; however, they could not all be included in the current study. To further improve implementation of preventive interventions, the aim for future research could be on studying factors that determine help seeking behavior, to get more insight in the concept of help seeking behavior among employees. Another possibility could be to focus on those employees who do experience health complaints and to study which factors have an influence on actual participation, in order to optimize participation in preventive intervention.

\section{Acknowledgements}

This study was financially supported by the Netherlands Organization for Health Research and Development (Zorg Onderzoek Nederland), grant no. 62200024, by CAPHRI School for Public Health and Primary Care, Maastricht, the Netherlands and by the Occupational Health Services Beter (trade name of ABN AMRO Arbo Services B.V.), Amsterdam, the Netherlands. 


\section{References}

1. Noordik E, van Dijk FJ, Nieuwenhuijsen K, van der Klink JJ. Effectiveness and cost-effectiveness of an exposurebased return-to-work programme for patients on sick leave due to common mental disorders: design of a cluster-randomized controlled trial. BMC Public Health. 2009;9:140.

2. Bultmann U, Sherson D, Olsen J, Hansen CL, Lund T, Kilsgaard J. Coordinated and tailored work rehabilitation: a randomized controlled trial with economic evaluation undertaken with workers on sick leave due to musculoskeletal disorders. J Occup Rehabil. 2009;19:81-93.

3. van der Klink JJ, Blonk RW, Schene AH, van Dijk FJ. Reducing long-term sickness absence by an activating intervention in adjustment disorders: a cluster randomised controlled design. Occup Environ Med. 2003;60:429-37.

4. van Oostrom SH, Anema JR, Terluin B, Venema A, de Vet HC, van Mechelen W. Development of a workplace intervention for sick-listed employees with stress-related mental disorders: Intervention Mapping as a useful tool. BMC Health Serv Res. 2007;7:127.

5. Brouwers EP, Tiemens BG, Terluin B, Verhaak PF. Effectiveness of an intervention to reduce sickness absence in patients with emotional distress or minor mental disorders: a randomized controlled effectiveness trial. Gen Hosp Psychiatry. 2006;28:223-9.

6. Huibers MJ, Beurskens AJ, Van Schayck CP, Bazelmans E, Metsemakers JF, Knottnerus JA, et al. Efficacy of cognitive-behavioural therapy by general practitioners for unexplained fatigue among employees: randomised controlled trial. Br J Psychiatry. 2004;184:240-6.

7. Janssen N, van den Heuvel WP, Beurskens AJ, Nijhuis FJ, Schroer CA, van Eijk JT. The demand-control-support model as a predictor of return to work. Int J Rehab Res. 2003;26:1-9.

8. Lander F, Friche $\mathrm{C}$, Tornemand $\mathrm{H}$, Andersen JH, Kirkeskov L. Can we enhance the ability to return to work among workers with stress-related disorders? BMC Public Health. 2009;9:372.

9. Kant I, Jansen NW, van Amelsvoort LG, van Leusden R, Berkouwer A. Structured early consultation with the occupational physician reduces sickness absence among office workers at high risk for long-term sickness absence: a randomized controlled trial. J Occup Rehabil. 2008;18:79-86.

10. Kant IJ, Jansen NW, van Amelsvoort LG, Swaen GM, van Leusden R, Berkouwer A. Screening questionnaire Balansmeter proved successful in predicting future long-term sickness absence in office workers. J Clin Epidemiol. 2009;62:408-14.

11. Judd F, Komiti A, Jackson $\mathrm{H}$. How does being female assist help-seeking for mental health problems? Aust $\mathrm{N}$ Z J Psychiatry. 2008;42:24-9.

12. Mackenzie CS, Scott T, Mather A, Sareen J. Older adults' help-seeking attitudes and treatment beliefs concerning mental health problems. Am J Geriatr Psychiatry. 2008;6:1010-9.

13. Rudell K, Bhui K, Priebe S. Do 'alternative' help-seeking strategies affect primary care service use? A survey of help-seeking for mental distress. BMC Public Health.2008;8:207.

14. Becker MH. Health belief model and personal health behavior. Health Educ Monogr. 1974;2:324-473.

15. Rosenstock IM. Why people use health services. Milbank Mem Fund Q. 1966;44:94-127.

16. KasI SV, Cobb S. Health behavior, illness behavior, and sick role behavior. Arch Environ Health. 1966;12:246-66. 
17. Mechanic D. The concept of illness behavior. J Chronic Dis. 1962;15:189-94.

18. Shaw, C. A framework for the study of coping, illness behaviour and outcomes. J Adv Nurs. 1999;29:124655.

19. Shaw C, Brittain K, Tansey R, Williams K. How people decide to seek health care: a qualitative study. Int J Nurs Stud. 2008;45:1516-24.

20. Wikman A, Marklund S, Alexanderson K. Illness, disease, and sickness absence: an empirical test of differences between concepts of ill health. J Epidemiol Community Health. 2005;59:450-4.

21. Mechanic D. Sociological dimensions of illness behavior. Soc Sci Med. 1995;41:1207-16.

22. Zola IK. Pathways to the doctor: from person to patient. Soc Sci Med. 1973;7:677-89.

23. Lexis MA, Jansen NW, van Amelsvoort LG, van den Brandt PA, Kant I. Depressive complaints as a predictor of sickness absence among the working population. J Occup Environ Med. 2009;51:887-95.

24. Kant IJ, Bultmann U, Schroer KA, Beurskens AJ, Van Amelsvoort LG, Swaen GM. An epidemiological approach to study fatigue in the working population: the Maastricht Cohort Study. Occup Environ Med. 2003;60:329.

25. Snaith RP. The Hospital Anxiety And Depression Scale. Health Qual Life Outcomes. 2003;1:29.

26. Zigmond AS, Snaith RP. The hospital anxiety and depression scale. Acta Psychiatr Scand. 1983;67:361-70.

27. Spinhoven P, Ormel J, Sloekers PP, Kempen GI, Speckens AE, Van Hemert AM. A validation study of the Hospital Anxiety and Depression Scale (HADS) in different groups of Dutch subjects. Psychol Med. 1997;27:363-70.

28. Andrea H, Bultmann U, Beurskens AJ, Swaen GM, van Schayck CP, Kant IJ. Anxiety and depression in the working population using the HAD Scale--psychometrics, prevalence and relationships with psychosocial work characteristics. Soc Psychiatry Psychiatr Epidemiol. 2004;39:637-46.

29. Karasek R, Brisson C, Kawakami N, Houtman I, Bongers P, Amick B. The Job Content Questionnaire (JCQ): an instrument for internationally comparative assessments of psychosocial job characteristics. J Occup Health Psychol. 1998;3:322-55.

30. Karasek RA. The Job Content Questionnaire and User's Guide (version 1.1). Los Angeles, CA: Department of Industrial and Systems Engineering, University of Southern California, 1985.

31. Hannay DR. The 'iceberg' of illness and 'trivial' consultations. J R Coll Gen Pract. 1980;30:551-4.

32. Mechanic, D. Health and illness behavior and patient-practitioner relationships. Soc Sci Med. 1992;34:134550.

33. Moller-Leimkuhler AM. Barriers to help-seeking by men: a review of sociocultural and clinical literature with particular reference to depression. J Affect Disord. 2002;71:1-9.

34. Raviv A, Sills R, Raviv A, Wilansky P. Adolescents' help-seeking behaviour: the difference between self- and other-referral. J Adolesc. 2000;23:721-40.

35. Hensing G, Alexanderson K, Allebeck P, Bjurulf P. Sick-leave due to psychiatric disorder: higher incidence among women and longer duration for men. Br J Psychiatry. 1996;169:740-6.

36. Mojtabai R. Social comparison of distress and mental health help-seeking in the US general population. Soc Sci Med. 2008;67:1944-50.

37. Jansen N, Kant I, van Amelsvoort L, Nijhuis F, van den Brandt P. Need for recovery from work: evaluating short-term effects of working hours, patterns and schedules. Ergonomics. 2003;46:664-80. 


\section{Prevention of long-term sickness absence and major depression in high risk employees: a randomized controlled trial}

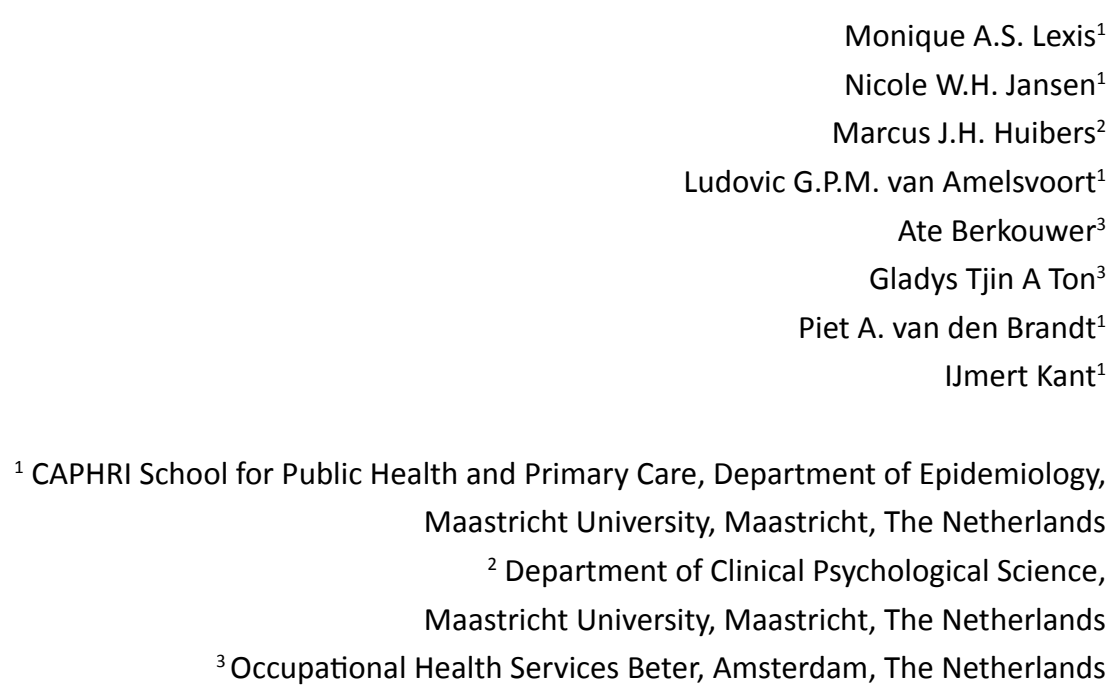

Occupational and Environmental Medicine, in press 


\section{Abstract}

\section{Objective}

To examine the efficacy of early intervention on the prevention of long-term sickness absence and major depression among employees at high risk of future sickness absence and with mild to severe depressive complaints.

\section{Methods}

Randomized controlled trial conducted among employees working in an office environment. 139 employees were identified both at high risk of future sickness absence and with mild to severe depressive complaints through screening. Subsequently, they were randomly assigned to the intervention group $(n=69)$ or the control group $(n=70)$. Objective sickness absence was analyzed at 12 and 18 months of follow-up. Depressive complaints were assessed by the Beck Depression Inventory (BDI-II) at baseline, 6 and 12 months of follow-up.

\section{Results}

Intention-to-treat analyses showed a significant difference in total sickness absence duration between the intervention (27.5 calendar days (SD 44.7)) and control group (50.8 days (SD 75.8)) over 12 months of follow-up, a reduction of $46 \%$ ( $p=0.017)$. The intervention group showed a nonsignificantly lower proportion of long-term sickness absence spells compared with the control group $(p=0.127)$. Statistically significant and clinically relevant differences in depressive complaints were found after both $6(p=0.001)$ and $12(p=0.005)$ months of follow-up, in favor of the intervention group. Relative risk reductions (RRR) were $19.2 \%$ and $19.8 \%$ respectively. Sickness absence data were available for all participants over 18 months of follow-up. Questionnaire data were available for 99 (at 6 months) and 90 participants (at 12 months). No adverse events or side effects occurred.

\section{Conclusions}

Early intervention in employees with mild to severe depressive complaints and high risk of future long-term sickness absence proved to be effective in preventing/reducing both sickness absence and depressive complaints. 


\section{Introduction}

Mental health problems are highly prevalent in the labor force and constitute a source of marked distress and social impairment for the employee. ${ }^{1,2}$ Major depression is considered to be one of the more severe disorders, making up a substantial part of the mental health problems. ${ }^{3}$ Recent studies showed that depressive complaints are also prevalent in the working population on a relatively mild level. ${ }^{4,5}$ Over time, these depressive complaints may exacerbate and result in major depression. ${ }^{6}$ Depressive complaints are found to be often associated with work disability and long-term sickness absence and increased healthcare use. ${ }^{7,8}$ Work disability and especially long-term sickness absence have also increasingly been recognized as considerable public health problems, with consequences for employers and society. ${ }^{9}$ So far, work rehabilitation in case of sickness absence due to mental health complaints remains very difficult and symptom reduction does not necessarily lead to work resumption. ${ }^{10,11}$ Conversely, sickness absence can cause and predict depressive complaints. ${ }^{12,13}$

From an occupational health care perspective, before treating employees with major depression or treatment of employees who are already on sick leave, a preventive strategy aimed at prevention of major depression and long-term sickness absence through early intervention might be more effective. A prerequisite for such a strategy is the ability to identify employees at high risk of future long-term sickness absence and with mild depressive complaints. To identify employees at high risk of sickness absence a screening questionnaire, called Balansmeter in Dutch, was developed in 1998. ${ }^{14}$ The Balansmeter proved to have good properties in predicting long-term sickness absence. In an additional study by Kant et al..$^{5}$ conducted in 2003 , it was demonstrated that early intervention among employees at risk of sickness absence proved effective in reducing future sickness absence. The current study, including a new RCT, focused on both high risk of sickness absence and depressive complaints. It was hypothesized that early intervention is effective in preventing depressive complaints and/or future sickness absence. Numerous studies have investigated the effectiveness of different treatments for depression. ${ }^{15,16}$ Strong evidence exists that cognitive behavioral therapy (CBT) and problem-solving therapy (PST) are effective treatments for depression. ${ }^{17-20}$ CBT and PST have also been proven effective in reducing sickness absence. ${ }^{21,22} \mathrm{~A}$ new protocol specifically targeted at employees, who are identified both at high risk of sickness absence and with mild to severe depressive complaints, was developed based on CBT and PST with the main focus on work-related problems. The aim of this RCT is to examine the efficacy of this new protocol among employees identified at high risk of long-term sickness absence and with mild to severe depressive complaints on the prevention of future long-term sickness absence and major depression. 


\section{Methods}

\section{Study design, procedure and participants}

A randomized controlled trial (RCT) was conducted among employees of a large banking company in the Netherlands. Figure 1 presents the selection process of the study. In 2007, 23,973 employees received a screening questionnaire at their home address. In the invitation letter, employees were asked to provide written informed consent, which covered the usage of questionnaire data, the company data on sickness absence and the possibility of participating in the trial. In total, 9,157 employees (38.2\%) responded to the questionnaire. 8,892 questionnaires were suitable for analyses, 265 were discarded due to technical reasons or because no informed consent was provided. Employees identified at risk of sickness absence and with mild to severe depressive complaints ( $n=211$ ) received an extensive baseline questionnaire (T0). Exclusion criteria were being (fully or partially) absent from work, pregnant or on pregnancy/maternity leave or receiving treatment by a psychologist/psychiatrist, at the time of completing the screening questionnaire. Employees who responded to the baseline questionnaire $(n=150)$ and still met the selection criteria $(n=139)$ were randomized into the intervention ( $n=69$ ) or the control group $(n=70)$. Randomization sequences were generated using computerized random number generators (block size 2 ) by the principal investigator $(\mathrm{ML})$, based on employee personnel numbers. Company counselors and psychologists were not blind to group allocation. However, they were only aware of participants of the intervention group. Followup questionnaires were sent at 6 and 12 months after randomization to assess depressive complaints and secondary outcomes. Objective sickness absence data were assessed at 12 and 18 months of follow-up. The study was covered by the medical guidelines of the occupational health services, thereby ensuring all participants' privacy and anonymity. The study was approved by the Medical and Ethical Committee of Maastricht University (MEC 06-3-018). The study was performed according to the Declaration of Helsinki.

\section{Preventive intervention}

The intervention consisted of a psychological treatment based on principles of PST and CBT. The main goal of the intervention, which had an individual orientated approach, was to offer guidelines to enhance problem solving skills in order to prevent long-term sickness absence and to reduce depressive complaints and to stimulate personal well-being. The intervention was specifically developed for our target population by four experts in the field of psychology. A treatment protocol and workbooks for practical assignments were developed for the trial, based on two main sources. ${ }^{23-26}$ The focus of the protocol was on employees with relatively mild complaints who are still at work. The intervention was preventive because of its timing, that is treatment before sickness absence occurs and because it focused on relatively mild complaints. Therefore, the intervention consisted largely of PST. CBT and PST often consist of 10 to 12 sessions. This new intervention consisted of a basic part and a specific part. The basic part contained 7 sessions of 45 minutes each, based on the major steps of PST. Through all sessions, principles of CBT were applied. The seventh session consisted of an 
Figure 1 Flow diagram of recruitment, allocation and outcome assessment

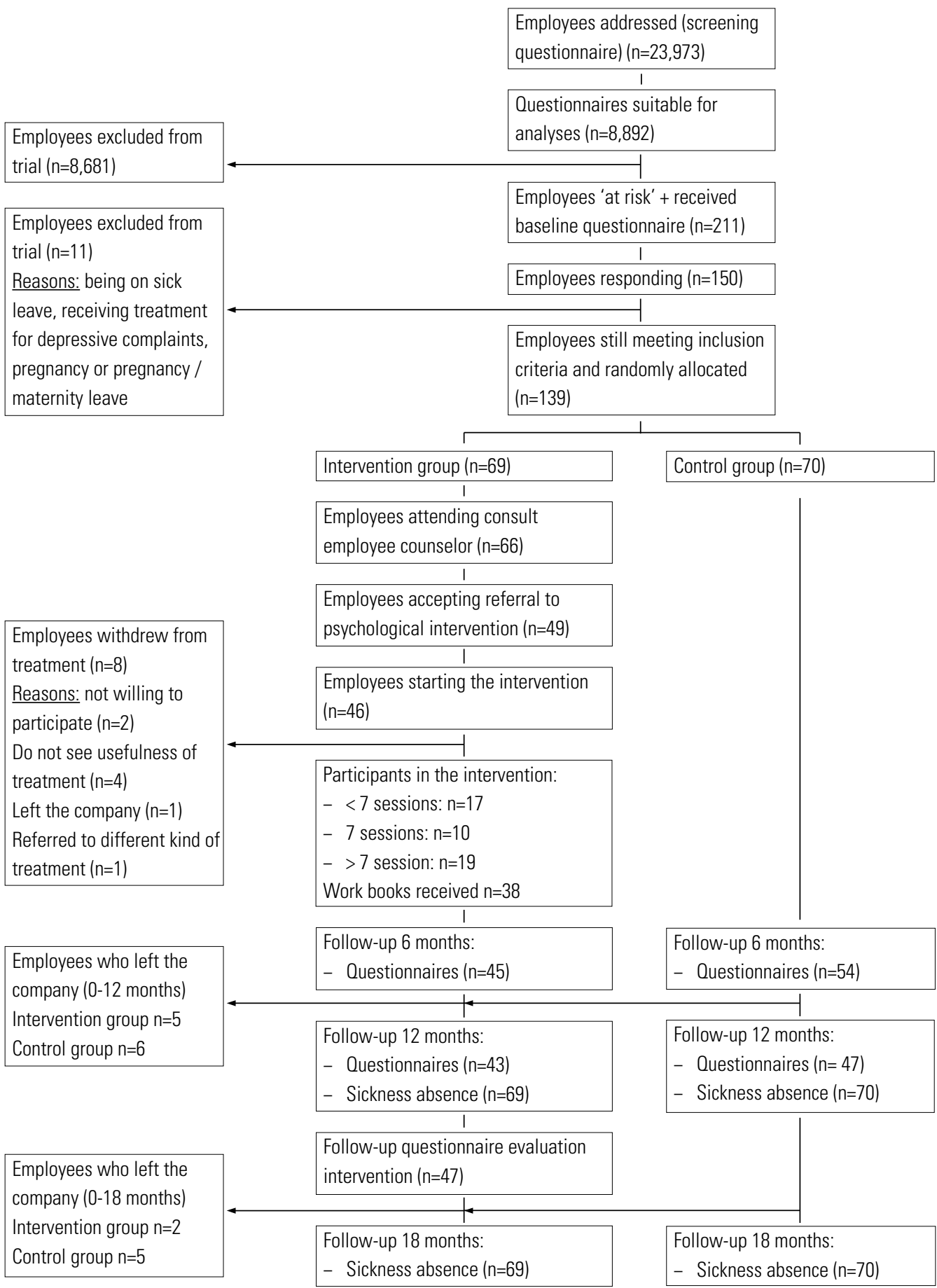


evaluation session. Since it was unknown at the start of the study if seven sessions would be sufficient for every participant, the intervention could be extended with a maximum of five sessions. In the seventh session, the psychologist and employee decided in consultation to end treatment in case the participant had recovered or to move on with the specific part of the protocol. For the specific part, the employee could indicate the subject to focus on during the sessions, such as training of social skills or cognitive restructuring. At the end of each session, homework assignments were given which were discussed in the next session. The intervention was conducted by 10 psychologists from a company consisting of a nationwide network of registered psychologists (Cenzo BV). This company regularly provides psychological healthcare for the banking company. The psychologists received 2 days of training before the study started and a 1-day booster session during the study. Participants in the control group received care as usual from the occupational health services. Care as usual when the employee asks for help included consultation with an occupational physician and, if necessary, referral to other disciplines. Care as usual in case of sickness absence included social medical counseling.

\section{Measurements}

\section{Screening measures}

\section{Risk of sickness absence}

The Balansmeter, developed in 1998 for employees working in an office work environment, was used to identify employees at high risk for future long-term sickness absence. The Balansmeter was developed and internally validated on data of the Maastricht Cohort Study and was externally validated in 2003 on a large sample of employees of the same company the current RCT was conducted at. ${ }^{14}$ For the present study a cut-off point with $87.9 \%$ specificity and $52.6 \%$ sensitivity in women and $87.8 \%$ and $65.1 \%$ in men was applied. The BM includes risk of sickness absence in general, not necessarily due to depressive complaints. A second measure was included to assess depressive complaints.

\section{Depressive complaints}

Presence and severity of depressive complaints were assessed using the depression scale of the Hospital Anxiety and Depression Scale (HAD Scale). The HAD Scale is a 14 item self-report questionnaire, that was originally developed to indicate the presence and severity of both anxiety (HAD-A) and depression (HAD-D) separately. ${ }^{27,}{ }^{28}$ With respect to the cut-off values, we used the values suggested by Zigmond et al. ${ }^{28} A$ higher score on HAD-D indicates more severe depressive complaints. Employees were defined as having mild to severe depressive complaints when they scored 8 points or higher on HAD-D. 


\section{Primary outcome measures}

\section{Sickness absence duration}

Sickness absence duration was measured objectively (in calendar days) through record linkage on an individual level with the company registers on certified sickness absence from 2 months after employees received the screening questionnaire until 18 months of follow-up. Long-term sickness absence was defined as a sickness absence spell lasting for more than 28 consecutive days. Sickness absence was assessed for 12 and 18 months of follow-up. Measurement of sickness absence started 2 months after completing the screening questionnaire. This is in line with the development of the Balansmeter. ${ }^{14}$ Returning and processing of the questionnaires could at most take 2 months.

\section{Depressive complaints}

Depressive complaints are viewed as a continuous spectrum of complaints, ranging from no to severe depressive complaints, with major depression at the end of the spectrum. While the screening instrument included the HAD Scale, to assess severity of depressive complaints more extensively the commonly used Beck Depression Inventory-II (BDI-II) was used. ${ }^{29}$ For our focus on relatively mild complaints, the BDI-II was too extensive and included too 'heavy' items, on for example suicidal thoughts, to include in the screening questionnaire. We therefore included the BDI-II in the baseline questionnaire and at 6 and 12 months of follow-up. Scores on the BDI-II can range from 0 to 63 , with higher scores indicating more severe depressive symptoms. ${ }^{30}$ Scores from 0 to 13 points were defined as not/minimally depressed, 14-19 points as possibly/lightly depressed, 20-28 as moderately depressed and 29-63 points as severely depressed. ${ }^{29,31}$

\section{Secondary outcome measures}

Data on secondary outcome measures were gathered by means of self-administered questionnaires, at baseline, 6 and 12 months of follow-up.

\section{Self-rated health}

Self-rated health was assessed with one item from the Short Form Health Survey (SF-36), widely used to measure general health status. ${ }^{32,33}$ 'In general, would you say your health is'. This item was scored on a five-point scale (1=excellent, $2=$ very good, $3=$ good, $4=$ moderate, $5=$ bad). The Brief Symptom Inventory (BSI), a short version of the Symptom Checklist 90 (SCL-90), was used to measure general psychological distress, with higher scores indicating a higher degree of symptoms (range 0-212). ${ }^{34,35}$ Scores on HAD-D at 6 and 12 months of follow-up were defined as a secondary outcome measure.

\section{Work characteristics}

A validated Dutch version of the Job Content Questionnaire (JCQ) was used to measure psychological job demands, decision latitude and social support at work. ${ }^{36}$ Additional data on job insecurity and 
commitment were gathered with questions adapted from the Questionnaire on the Experience and Evaluation of Work (VBBA). ${ }^{37}$

\section{Co-interventions}

Co-interventions in the past 6 months, such as treatment by a general practitioner, an occupational physician, a psychologist, a psychiatrist or a company counselor and the use of medication (including anti-depressants, sleeping medication, tranquillizers, painkillers or others) were assessed by selfreport.

\section{Treatment adherence}

For each participant in the intervention group, it was assessed whether the intervention was conducted according to the protocol. Adherence to the protocol was defined as being exposed to all essential predefined steps of the intervention. For the per-protocol analyses, those participants who received at least one treatment session were included, which is a rather conservative approach.

\section{Power calculation}

In calculating sample size, sickness absence was chosen as the main outcome measure of the study. Based on sickness absence data from the Maastricht Cohort Study on fatigue at work, it appeared that $60 \%$ of employees with psychological complaints were absent from work for at least 2 weeks over a period of 12 months of follow-up. ${ }^{2}$ The assumption was that the intervention would be effective by reducing this percentage to $35 \%$. Power calculation, using a two-sided significance level of $5 \%$ and a power of $80 \%$, indicated a minimum of 59 participants in each group. Taking into account a potential attrition of $15 \%$, a final sample size of 136 should be sufficient.

\section{Statistical analysis}

First, data were analyzed according to the intention-to-treat principle. Difference scores on continuous outcomes were used for both sickness absence (0-12 months and 0-18 months) and depressive complaints (TO-T1 and TO-T2). To estimate the efficacy of the intervention, Poisson regression analysis was used for the outcomes sickness absence duration and sickness absence frequency. Risk ratios were calculated for dichotomous outcomes. Linear regression analysis was used for continuous outcomes, adjusted for baseline differences. Multivariate Cox regression analysis was used to test differences in time to onset of the first sickness absence spell. Clinically meaningful changes on the BDI-II were determined by calculating the Reliable Change Index as developed by Jacobson and Truax. ${ }^{38}$ This approach is based on two components: reliable change, that is a decrease of at least 7 points; and clinically significant change, that is a post-treatment score below $14 . .^{29,31}$ Chi-square tests were used to test frequency differences in reliable and clinically significant change.

Second, for the per-protocol analyses, outcomes were compared between those employees who received at least one treatment session and the control group.

All analyses were performed using SPSS version 15.0, Stata statistical software package 8.0 and SAS. 


\section{Results}

Table 1 Baseline characteristics of the trial population

\begin{tabular}{|c|c|c|}
\hline Variable & $\begin{array}{l}\text { Intervention group } \\
(\mathrm{n}=69)\end{array}$ & $\begin{array}{l}\text { Control group } \\
(\mathrm{n}=70)\end{array}$ \\
\hline Gender, male; N (\%) & $42(60.9)$ & $43(61.4)$ \\
\hline Age (18-65); mean (SD) & $48.41(8.68)$ & $47.07(9.49)$ \\
\hline \multicolumn{3}{|l|}{ Highest level of education; $\mathrm{N}(\%)$ * } \\
\hline Low & $5(7.9)$ & $6(9.0)$ \\
\hline Medium & $49(77.8)$ & $45(67.2)$ \\
\hline High & $9(14.3)$ & $16(23.9)$ \\
\hline Presence of long-term illness; N (\%) † & $39(59.1)$ & $34(51.5)$ \\
\hline Living alone; $N(\%) \dagger$ & $12(17.4)$ & $13(18.6)$ \\
\hline Depressive complaints (HAD-D) (0-21) ‡; mean (SD) & $10.45(2.67)$ & $9.97(2.34)$ \\
\hline Depressive complaints (BDI-II) (0-63) ‡; mean (SD) & $17.03(9.56)$ & $14.84(8.11)$ \\
\hline Psychological distress (BSI) (0-212) ‡; mean (SD) & $40.79(27.85)$ & $35.34(25.47)$ \\
\hline $\begin{array}{l}\text { Score on Balansmeter (indicating risk of sickness absence); } \\
\text { mean (SD) }\end{array}$ & -0.5902 (0.9599) & $-0.5993(0.7562)$ \\
\hline Working hours / week; mean (SD) & $34.8(4.52)$ & $34.9(4.91)$ \\
\hline Having an executive position; $\mathrm{N}(\%) \dagger$ & $4(5.8)$ & $7(10.0)$ \\
\hline
\end{tabular}

* Categorical variable with $\mathrm{N}(\%)$ indicating 'yes'; † dichotomous variable (yes / no) with $\mathrm{N}(\%)$ indicating 'yes'; † scale range'.

\section{Participants}

Figure 1 presents a flow chart of the study. Recruitment started in February 2007 and was completed in October 2007. A total of 139 employees were enrolled in the trial and randomized into the intervention group ( $n=69$ ) or the control group $(n=70)$. At 6 and 12 months of follow-up, questionnaire data were available for 99 and 89 participants respectively. Objective sickness absence data were available for all participants for 18 months of follow-up. The baseline characteristics of the trial population are listed in table 1. Except for BDI-II and BSI, baseline characteristics were comparable between the intervention and control group.

\section{Effects of the intervention on the primary outcomes sickness absence duration and depressive complaints}

Table 2 presents the results of the intention-to-treat analysis for the primary outcome measures sickness absence duration at 12 and 18 months of follow-up and depressive complaints at 6 and 12 months of follow-up. A significant difference in total sickness absence duration was found between the intervention ( 27.5 days) and control group (50.8 days) at 12 months of follow-up, a reduction of $46 \%(p=0.017)$. The intervention group showed a non-significantly lower proportion of long-term sickness absence spells (nine of 69) compared with the control group (17 of 70) ( $p=0.127$ ) at 12 months of follow-up. At 18 months of follow-up, the total sickness absence duration was 45.0 days in 
Table 2 Outcomes of the intention-to-treat analysis, with objective sickness absence measured at 12 months and 18 months of follow-up and questionnaire data measured at baseline (TO), 6 months (T1) and 12 months (T2) of follow-up

\begin{tabular}{|c|c|c|c|}
\hline Variable & $\begin{array}{l}\text { Intervention group } \\
(\mathrm{n}=69)\end{array}$ & $\begin{array}{l}\text { Control group } \\
(n=70)\end{array}$ & $\begin{array}{l}\text { ß, RR, HR § } \\
(95 \% \mathrm{Cl}) ; p \text {-value }\end{array}$ \\
\hline \multicolumn{4}{|l|}{ Objective sickness absence } \\
\hline \multicolumn{4}{|l|}{$0-12 *$} \\
\hline At least one time on sick leave; $\mathrm{N}(\%) \ddagger$ & $56(81.2)$ & $48(68.6)$ & $1.18(0.97-1.44) ; 0.118$ \\
\hline $\begin{array}{l}\text { Duration (calendar days); mean (SD) / } \\
\text { median }\end{array}$ & $\begin{array}{l}27.48(44.74) / \\
11.00\end{array}$ & $\begin{array}{l}50.83(75.75) / \\
15.00\end{array}$ & $-0.62(-1.12--0.11) ; 0.017$ \\
\hline Frequency; mean (SD) / median & $2.34(1.61) / 2.00$ & $2.35(1.78) / 2.00$ & $-0.01(-0.28-0.26) ; 0.963$ \\
\hline $\begin{array}{l}\text { Time to onset of first sickness absence } \\
\text { spell (calendar days); mean (SD) }\end{array}$ & 151.38 (136.99) & $187.37(146.42)$ & $1.35(0.92-1.99) ; 0.129$ \\
\hline \multicolumn{4}{|l|}{$0-18 \dagger$} \\
\hline At least one time on sick leave; $\mathrm{N}(\%) \ddagger$ & $62(89.9)$ & $56(80.0)$ & $1.12(0.98-1.29) ; 0.154$ \\
\hline $\begin{array}{l}\text { Duration (calendar days); mean (SD) / } \\
\text { median }\end{array}$ & $\begin{array}{l}45.03(76.59) / \\
20.50\end{array}$ & $\begin{array}{l}62.57(81.89) / \\
25.50\end{array}$ & $-0.33(-0.78-0.12) ; 0.150$ \\
\hline Frequency; mean (SD) / median & $2.97(2.09) / 2.00$ & $2.93(2.17) / 2.00$ & $0.01(-0.24-0.67) ; 0.918$ \\
\hline $\begin{array}{l}\text { Time to onset of first sickness absence } \\
\text { spell (calendar days); mean (SD) }\end{array}$ & $177.87(184.21)$ & $231.19(206.57)$ & 1.34 (0.93-1.93); 0.117 \\
\hline \multicolumn{4}{|l|}{$\begin{array}{l}\text { Participants with sickness absence } \\
\text { spell > } 28 \text { calendar days; } N(\%) \ddagger\end{array}$} \\
\hline $0-12 *$ & $9(13.0)$ & $17(24.6)$ & $0.54(0.26-1.12) ; 0.127$ \\
\hline $0-18 \dagger$ & $14(20.3)$ & $22(31.4)$ & $0.65(0.36-1.16) ; 0.175$ \\
\hline
\end{tabular}

Depressive complaints according to

BDI-II, (0-63) १; Mean (SD)

\begin{tabular}{llll} 
Baseline T0 & $17.03(9.55)$ & $14.84(8.11)$ & \\
T1 & $12.77(10.48)$ & $16.30(10.08)$ & \\
T2 & $12.42(9.64)$ & $16.69(11.04)$ & \\
T1-T0 & $-4.41(7.00)$ & $0.92(7.06)$ & $-5.08(-7.91--2.25) ; 0.001$ \\
T2-T0 & $-3.79(8.45)$ & $2.09(9.51)$ & \\
\cline { 2 - 3 } Depressive complaints according to & & & \\
HAD-D, (0-21) 9; Mean (SD) & & & \\
Screening & $10.45(2.67)$ & $9.97(2.34)$ & $-1.68) ; 0.005$ \\
T1 & $7.67(4.26)$ & $8.55(3.81)$ & \\
T2 & $7.24(4.62)$ & $9.40(4.23)$ & \\
T1-Screening & $-2.91(3.67)$ & $-1.45(3.06)$ & $-1.38(-2.74--0.02) ; 0.046$ \\
T2-Screening & $-3.24(4.50)$ & $-0.38(3.91)$ & $-2.62(-4.41--0.83) ; 0.005$
\end{tabular}


Psychological distress (BSI),

(0-212) १; Mean (SD)

Baseline

$40.79(27.85) \quad 35.34(25.47)$

$\mathrm{T} 1$

32.26 (30.84)

$36.89(29.43)$

T2

32.19 (33.29)

42.19 (33.78)

T1-T0

$-9.71(18.12)$

$4.55(17.33)$

T2-TO

$-9.32(25.39)$

$6.51(15.69)$

$-13.88(-21.72--6.04) ; 0.001$

$-15.69(-25.11--6.26) ; 0.001$

* Measured from baseline until 12 months of follow-up; $†$ measured from baseline until 18 months of follow-up; $\ddagger$ dichotomous variable (no / yes) with N (\%) indicating 'yes'; § ß $(95 \% \mathrm{CI})$ in case of continuous variable, risk ratio $(95 \% \mathrm{Cl})$ in case of dichotomous variable and $\mathrm{HR}$ in case of time to onset of first sickness absence spell; १ scale range.

the intervention group and 62.6 days in the control group, a reduction of $28.0 \%$. However, this result failed to reach statistical significance $(p=0.150)$. No significant difference was found in the proportion of long-term sickness absence as well $(p=0.175)$. Regarding depressive complaints, significant differences in change scores were observed for both T1-T0 $(p=0.001)$ and T2-T0 $(p=0.005)$ between the intervention and control group, in favor of the intervention group. Depressive complaints decreased in the intervention group during follow-up and increased in the control group. The intervention group showed a significantly higher proportion of participants with a reliable change in depressive complaints ( 15 of 43 ) versus the control group ( 8 of 51) at $6(p=0.031$ ) and 12 months $(p=0.022$ ) of follow-up (proportion reliable change in the intervention group 13 of 42 , in the control group 5 of 45 ). RRR were $19.2 \%$ and $19.8 \%$, NNT were 5.2 (95\% Cl 2.7 to 55.5$)$ and $5.0(95 \% \mathrm{Cl} 2.7$ to 32.1) respectively, for 6 and 12 months of follow-up. The proportion of clinically relevant change in the intervention group was 27 of 44 versus 26 of 53 in the control group at 6 months of follow-up $(p=0.225)$. At 12 months, clinically relevant change in the intervention group was 28 of 43 and 20 of 45 in the control group ( $p=0.052)$.

\section{Effects of the intervention on secondary outcomes}

Table 2 also presents the results of the intention-to-treat analysis for the secondary outcomes. Comparable with the results on BDI-II, statistically significant differences in change scores on HAD-D were found in favor of the intervention group, between T1-T0 and T2-T0. Significant differences were found for change scores for T1-T0 and T2-T0 on BSI as well. Again, scores decreased in the intervention group and increased in the control group. No significant differences were found between T1-T0 and T2-TO between both groups on self-rated health and work characteristics (results not shown). 
Table 3 Outcomes of the per-protocol analysis, objective sickness absence measured at 12 and 18 months of follow-up and questionnaire data measured at baseline (T0), 6 (T1) and 12 months (T2) of follow-up

\begin{tabular}{|c|c|c|c|}
\hline $\begin{array}{l}\text { Variable } \\
\text { n }\end{array}$ & $\begin{array}{l}\text { Intervention group, } \\
\text { 'never started' } \\
\text { group excluded } \\
(\mathrm{n}=46)\end{array}$ & $\begin{array}{l}\text { Control group } \\
(n=70)\end{array}$ & $\begin{array}{l}\text { B, RR, HR § } \\
(95 \% \mathrm{CI}) ; p \text {-value }\end{array}$ \\
\hline \multicolumn{4}{|l|}{ Objective sickness absence } \\
\hline \multicolumn{4}{|l|}{$0-12^{*}$} \\
\hline At least one time on sick leave; $\mathrm{N}(\%) \ddagger$ & $34(73.9)$ & $48(68.6)$ & $1.08(0.85-1.36) ; 0.677$ \\
\hline $\begin{array}{l}\text { Duration (calendar days); mean (SD) / } \\
\text { median }\end{array}$ & $24.29(34.18) / 11.00$ & $50.83(75.75) / 15.00$ & $-0.74(-1.37--0.11) ; 0.021$ \\
\hline Frequency; mean (SD) / median & $2.18(1.47) / 2.00$ & 2.35 (1.78) / 2.00 & $-0.08(-0.39-0.23) ; 0.621$ \\
\hline $\begin{array}{l}\text { Time to onset of first sickness absence } \\
\text { spell (calendar days); mean (SD) }\end{array}$ & $165.67(143.77)$ & $187.37(146.42)$ & $1.18(0.76-1.82) ; 0.473$ \\
\hline \multicolumn{4}{|l|}{$0-18 \dagger$} \\
\hline At least one time on sick leave; $\mathrm{N}(\%) \ddagger$ & $39(84.8)$ & $56(80.0)$ & $1.06(0.89-1.26) ; 0.625$ \\
\hline $\begin{array}{l}\text { Duration (calendar days); mean (SD) / } \\
\text { median }\end{array}$ & 33.05 (51.18) / 15.00 & $62.57(81.89) / 25.50$ & $-0.64(-1.18--0.10) ; 0.021$ \\
\hline Frequency; mean (SD) / median & $2.69(1.89) / 2.00$ & $2.93(2.17) / 2.00$ & $-0.08(-0.37-0.20) ; 0.569$ \\
\hline $\begin{array}{l}\text { Time to onset of first sickness absence } \\
\text { spell (calendar days); mean (SD) }\end{array}$ & 200.76 (198.97) & $231.19(206.57)$ & $1.18(0.78-1.77) ; 0.441$ \\
\hline \multicolumn{4}{|l|}{$\begin{array}{l}\text { Participants with sickness absence } \\
\text { spell > } 28 \text { calendar days; } N(\%) \ddagger\end{array}$} \\
\hline $0-12 *$ & $6(13.0)$ & $17(24.3)$ & $0.54(0.23-1.26) ; 0.160$ \\
\hline $0-18 \dagger$ & $7(15.2)$ & $22(31.4)$ & 0.48 (0.23- 1.04$) ; 0.078$ \\
\hline $\begin{array}{l}\text { Depressive complaints } \\
\text { according to } \\
\text { BDI-II, (0-63) १; Mean (SD) }\end{array}$ & & & \\
\hline Baseline TO & $18.15(9.42)$ & $14.84(8.11)$ & \\
\hline $\mathrm{T} 1$ & $14.00(11.50)$ & $16.30(10.08)$ & \\
\hline $\mathrm{T} 2$ & $12.31(10.01)$ & $16.69(11.04)$ & \\
\hline T1-T0 & $-4.50(7.59)$ & $0.93(7.06)$ & $-5.02(-8.34--1.70) ; 0.004$ \\
\hline T2-T0 & $-4.66(8.76)$ & $2.09(9.51)$ & $-6.05(-10.34--1.75) ; 0.006$ \\
\hline
\end{tabular}

* Measured from baseline until 12 months of follow-up; $†$ measured from baseline until 18 months of follow-up; $\ddagger$ dichotomous variable (no/yes) with $\mathrm{N}(\%)$ indicating 'yes'; § ß $(95 \% \mathrm{Cl})$ in case of continuous variable and risk ratio $(95 \% \mathrm{Cl})$ in case of dichotomous variable and HR in case of time to onset of first sickness absence spell; ๆ scale range. 


\section{Per-protocol analysis}

Table 3 presents the results of the per-protocol analyses. Sickness absence and depressive complaints were compared between the intervention group, including those employees who received at least one session, and the control group. The intervention group demonstrated a significantly lower sickness absence duration of $52.2 \%$ after 12 months ( $p=0.021)$ and $47.1 \%$ after 18 months $(p=0.021)$ compared with the control group. The latter result of sickness absence duration at 18 months of follow-up differs with the result of the intention-to-treat analysis, in which a non-significant reduction of $28.0 \%$ was found. No significant difference was found for the proportion of sickness absence spells $>28$ days at 12 months $(p=0.160)$ and 18 months $(p=0.078)$ of follow-up. Regarding depressive complaints, similar to the results of the intention-to-treat analysis, statistically significant lower scores on BDI-II were found between T1-TO $(p=0.004)$ and T2-TO $(p=0.006)$ in the intervention group. HAD-D also showed significantly lower scores at T2-Screening in the intervention group and for BSI significant differences were found between both T1-T0 and T2-T0 (results not shown).

Table 4 Co-interventions used 6 months before completing questionnaires T1 and T2

\begin{tabular}{|c|c|c|c|}
\hline Variable & $\begin{array}{l}\text { Intervention group } \\
(\mathrm{n}=69)\end{array}$ & $\begin{array}{l}\text { Control group } \\
(n=70)\end{array}$ & $\mathrm{p}$-value \\
\hline \multicolumn{4}{|c|}{ Co-interventions *; N (\%) } \\
\hline At T1 & $35(77.8)$ & 35 (64.8) & 0.158 \\
\hline At T2 & $31(72.1)$ & $33(70.2)$ & 0.960 \\
\hline \multicolumn{4}{|c|}{$\begin{array}{l}\text { Receiving treatment for depressive } \\
\text { complaints (not our intervention); } \mathrm{N}(\%)\end{array}$} \\
\hline At T1 & $2(4.4)$ & $7(13.0)$ & 0.142 \\
\hline At T2 & $5(11.6)$ & 4 (8.5) & 0.644 \\
\hline \multicolumn{4}{|c|}{ Medication use; N (\%) } \\
\hline At T1 & $33(73.3)$ & $35(64.8)$ & 0.363 \\
\hline At T2 & $27(62.8)$ & $27(57.4)$ & 0.788 \\
\hline
\end{tabular}

* Treatment by general practitioner, occupational physician, psychologist, psychiatrist, company counselor or others.

\section{Treatment received and co-interventions}

Of the 69 employees allocated to the intervention group, 38 employees completed the intervention and returned their workbooks. The mean number of sessions was 7.5 (SD 2.5). The mean protocol adherence of the psychologists was 95\% (SD 12.6). With respect to the use of co-interventions (table 4 ) only a small number of employees received co-interventions specifically for depressive complaints. No significant and clinically relevant differences were found between the intervention and control group at baseline, 6 and 12 months of follow-up in their use of co-interventions. 


\section{Discussion}

\section{Principal findings and interpretation of outcomes}

The aim of this study was to evaluate the effect of a preventive intervention among employees identified at high risk of future long-term sickness absence and with mild to severe depressive complaints in a RCT. The preventive intervention had a substantial effect on sickness absence duration and depressive complaints in favor of the intervention group. During the years in which this study was conducted, sickness absence rates in the company under study were $3.83 \%$ (in 2007 ) and $3.77 \%$ (in 2008). Sickness absence rates were $13.93 \%$ in the control group and $7.53 \%$ in the intervention group over 12 months of follow-up, which indicates that indeed a high-risk group was selected. Although sickness absence was substantially lower in the intervention group than in the control group, the sickness absence rates of the company could not be reached during the follow-up of 18 months. The proportion of long-term sickness absence spells showed no significant difference between the intervention and control group, despite a reduction of $40 \%$ as described in the power calculation. This may be explained by the decrease in sickness absence rates in the Netherlands, from approximately $6.0 \%$ in 1999 , the year in which the power calculation was conducted, to $4.3 \%$ in 2009 . Results may have failed to reach statistical significance due to the lower prevalence of sickness absence in the population. Sickness absence frequency and time to onset of the first sickness absence spell may have been influenced by the intervention as well, but no significant differences were found between the groups, which may have been because the power was too low.

With regard to depressive complaints (BDI-II), substantial effects were found in the intention-to-treat analysis, again in favor of the intervention group. Scores on BDI-II increased over time in the control group and decreased in the intervention group. Participants in the intervention and control group did not differ in their use of co-interventions. Co-interventions were not likely to have influenced the results, but this could not be completely ruled out. Co-interventions might have reduced the contrast between the groups, and this might have led to an underestimation of the effects found.

Regarding the per-protocol analysis, sickness absence duration was significantly lower in the intervention group at 12 but also at 18 months. These results indicate that when participants received at least one session, the reduction of sickness absence duration remained approximately $50 \%$ until 18 months of follow-up. Regarding depressive complaints, similar results to the intention-to-treat analysis were found. Again, scores on BDI-II increased in the control group and decreased in the intervention group over time. Thus, these results demonstrate that even a small number of sessions seems to be effective. For the per-protocol analysis, a rather conservative approach was used, which also indicates that our results may be an underestimation.

\section{Methodological considerations}

Strengths of the study include the randomized design, the availability of a nationwide study population of employees, the objective measurement of sickness absence, the intervention for 
which a specific protocol was developed, the long follow-up period of 18 months and the monitoring of co-interventions.

Some considerations should be taken into account when interpreting the results of the study. During the pilot study conducted prior to the RCT, a smaller than expected overlap between the concepts of risk of future sickness absence and depressive complaints was found. To guarantee sufficient numbers of participants, two adjustments to the original study design were required. First, the screening questionnaire was sent to more employees than originally planned. Second, the cut-off point on the Balansmeter was adapted to a cut-off point with a higher sensitivity to improve the overlap between the concepts. The original cut-off point on the HAD-D was maintained, since this cut-off point has been used in many studies. The smaller than expected overlap between both concepts may be explained by differences in the background of the concepts. Risk of sickness absence has a multi-factorial etiology, which does not necessarily include health complaints.

The HAD-D was used to identify employees with mild to severe depressive complaints. To study the course of depressive complaints, the more widely used and more extensive questionnaire BDI-II was used. Our results indicate that many of those employees identified with mild to severe depressive complaints by HAD-D (48.2\%) scored in the no to minimal depressive complaints range of the BDI-II at baseline. The average BDI-II score was 9.77 (SD 3.35) in this category. Regardless of the relatively low score of the employees identified, scores were still in the upper part of the lowest category of BDI-II (range 0-13). So, a reliable and relevant change on BDI-II (> 7 points) could still occur.

With regard to blinding, the researcher analyzed all data anonymously using personnel numbers. Participants in the RCT were not blinded, since they were aware into which group they were randomized. However, little influence on the results was expected, since no differences in the use of co-interventions were observed. Company counselors and psychologists were partially blinded, as they were only aware of participants in the intervention group. Therefore, no influence on the contrast between the intervention and control group was expected. Incomplete blinding may have had some influence on the outcome depressive complaints and the secondary outcomes. However, sickness absence was measured objectively and blinded for the researcher and could therefore not have influenced the results.

Only 38 employees of the 69 employees who were randomized into the intervention group received the intervention according to protocol, implying $44.9 \%$ incomplete interventions. A withdrawal rate of $35.3 \%$ was found on the questionnaires at 12 months of follow-up. Drop-outs and loss-to-follow-up in the intervention group could have affected the results. However, since objective sickness absence data were available for all 139 randomized employees, there were no consequences for this outcome. For depressive complaints, no selective attrition was found, and no differences in baseline values on BDI-II were found between respondents and non-respondents on T1 and T2. The high number of employees dropping out after consultation with the company counselor may be explained by the fact that the focus of the RCT was on relatively mild depressive complaints and an increased risk for a future event. Many employees reported not to experience health complaints at the moment of completing the screening questionnaire and therefore may have refused participation. ${ }^{39}$ 


\section{Implications and future research}

This study focused on prevention of sickness absence and major depression. However prevention will never completely compensate treatment, since there will always be employees who will develop a clinical depression, who have depressive complaints but do not lose their work ability, who will go on sick leave or who do not benefit from preventive intervention. As a result, treatment remains very important.

The focus of our RCT was on employees with relatively mild depressive complaints. Within this mild level, complaints vary in severity. Our results demonstrated that 17 of the 46 employees who started participation in the intervention received fewer than seven sessions of the basic part of the intervention. From a research perspective, it was preferable to indicate a fixed number of sessions the participants should receive in the intervention, to be able to exactly define completion of the intervention. However, in practice this approach was not feasible, since it was unknown how many sessions were required for the mild level of complaints. The per-protocol analysis showed that receiving even a small number of sessions seems already effective. This may indicate that the intervention should be tailored to an employee's individual level of complaints.

The efficacy of the preventive intervention was evaluated among employees in an office work environment. Therefore, this study is not fully representative for the general working population. Nevertheless, it is believed that the intervention may be suitable for similar companies in the occupational healthcare setting, because of the occurrence of comparable kinds of complaints/ problems, characterized by stress-related (mental) disorders.

When considering implementation of the preventive strategy into daily practice, employers and policymakers should weigh the effects against the costs of the intervention. Evaluation of the costeffectiveness was not part of the study. However, based on the intention-to-treat analysis a decrease of 555 working days of sickness absence between the intervention and control group was calculated over 18 months of follow-up. The average labor costs account for $€ 200$ per working day. Thus, the profits of reduction of sickness absence amounted to $€ 111,000$. Besides benefits in terms of money due to sickness absence, it is known that depression is associated not only with absenteeism but also with presenteeism. ${ }^{40}$ The positive effect of the intervention on depressive complaints may lead to increased at-work job performance and productivity. In future research, a more extensive costbenefit analysis should be performed including all facets of the preventive strategy.

In conclusion, the results of this study showed strong preventive effects on both long-term sickness absence and major depression. In the light of the difficult return to work in employees who are already on sick leave, this study clearly demonstrated that a new preventive intervention is a more promising approach in the prevention and reduction of sickness absence and improvement of mental health among employees. 


\section{Acknowledgements}

This study was funded by the Netherlands Organization for Health Research and Development, The Netherlands (grant no. 62200024), by CAPHRI School for Public Health and Primary Care, Maastricht, the Netherlands, and by the Occupational Health Services Beter (ABN AMRO Arbo Services), Amsterdam, the Netherlands. The authors thank A. Arntz and M. Huibers (Maastricht University), T. Bouman (University Medical Center Groningen) and A. van den Hout (Academic Community Health Centre Maastricht), experts in the field of psychology, for development of the protocol and workbooks for the preventive intervention and training of the participating psychologists. The authors also thank D. de Bruyn, S. Dijkstra, H. Kalter, W. ten Haaf and P. Batting, company counselors from Occupational Health Services Beter, for performing the consultations. The authors also thank I. Pos, director of Cenzo B.V., for coordination of execution of the intervention, and J. Biemans, S. Decoz, J. Haasnoot, A. Janssen, A. Kuipers, G. Langenhuizen, M. Ooms, G. Oppewal, R. Verhoog and M. Udenhout, psychologists from Cenzo B.V., for providing the psychological intervention. The authors thank J. Slangen and P. Brouns (Maastricht University), B. Couwenberg and M. Binsbergen (Occupational Health Services Beter), for their contribution to data management. 


\section{References}

1. Bultmann U, Kant I, KasI SV, et al. Fatigue and psychological distress in the working population: psychometrics, prevalence, and correlates. J Psychosom Res 2002;52:445-52.

2. Kant IJ, Bultmann U, Schroer KA, et al. An epidemiological approach to study fatigue in the working population: the Maastricht Cohort Study. Occup Environ Med 2003;60 Suppl 1:i32-9.

3. World Health Organization. World Health Report 2000. Geneva, Switzerland: World Health Organization, 2000 .

4. Andrea H, Bultmann U, Beurskens AJ, et al. Anxiety and depression in the working population using the $H A D$ Scale--psychometrics, prevalence and relationships with psychosocial work characteristics. Soc Psychiatry Psychiatr Epidemiol 2004;39:637-46.

5. Kant I, Jansen NW, van Amelsvoort LG, et al. Structured early consultation with the occupational physician reduces sickness absence among office workers at high risk for long-term sickness absence: a randomized controlled trial. J Occup Rehabil 2008;18:79-86.

6. Laitinen-Krispijn S, Bijl RV. Mental disorders and employee sickness absence: the NEMESIS study. Netherlands Mental Health Survey and Incidence Study. Soc Psychiatry Psychiatr Epidemiol 2000;35:71-7.

7. Lexis MA, Jansen NW, van Amelsvoort LG, et al. Depressive complaints as a predictor of sickness absence among the working population. J Occup Environ Med 2009;51:887-95.

8. Stansfeld S, Feeney A, Head J, et al. Sickness absence for psychiatric illness: the Whitehall II Study. Soc Sci Med 1995;40:189-97.

9. Marmot M, Feeney A, Shipley M, et al. Sickness absence as a measure of health status and functioning: from the UK Whitehall II study. J Epidemiol Community Health 1995;49:124-30.

10. Blank L, Peters J, Pickvance S, et al. A systematic review of the factors which predict return to work for people suffering episodes of poor mental health. J Occup Rehabil 2008;18:27-34.

11. Brouwers EP, Tiemens BG, Terluin B, et al. Effectiveness of an intervention to reduce sickness absence in patients with emotional distress or minor mental disorders: a randomized controlled effectiveness trial. Gen Hosp Psychiatry 2006;28:223-29.

12. Melchior M, Ferrie JE, Alexanderson K, et al. Using sickness absence records to predict future depression in a working population: prospective findings from the GAZEL cohort. Am J Public Health 2009;99:1417-22.

13. Vahtera J, Westerlund $\mathrm{H}$, Ferrie JE, et al. All-cause and diagnosis-specific sickness absence as a predictor of sustained sub-optimal health: a 14-year follow-up in the GAZEL cohort. J Epidemiol Community Health 2010;64:311-7.

14. Kant IJ, Jansen NW, van Amelsvoort LG, et al. Screening questionnaire Balansmeter proved successful in predicting future long-term sickness absence in office workers. J Clin Epidemiol 2009;62:408-14.

15. Gloaguen V, Cottraux J, Cucherat M, et al. A meta-analysis of the effects of cognitive therapy in depressed patients. J Affect Disord 1998;49:59-72.

16. Wampold BE, Minami T, Baskin TW, et al. A meta-(re)analysis of the effects of cognitive therapy versus 'other therapies' for depression. J Affect Disord 2002;68:159-65.

17. Beck AT. The current state of cognitive therapy: a 40-year retrospective. Arch Gen Psychiatry 2005;62:953-9. 
18. Hollon SD, Stewart MO, Strunk D. Enduring effects for cognitive behavior therapy in the treatment of depression and anxiety. Annu Rev Psychol 2006;57:285-315.

19. Bell AC, D'Zurilla TJ. Problem-solving therapy for depression: a meta-analysis. Clin Psychol Rev 2009;29:34853.

20. Mynors-Wallis LM, Gath DH, Day A, et al. Randomised controlled trial of problem solving treatment, antidepressant medication, and combined treatment for major depression in primary care. BMJ 2000;320:2630.

21. McCrone P, Knapp M, Proudfoot J, et al. Cost-effectiveness of computerised cognitive-behavioural therapy for anxiety and depression in primary care: randomised controlled trial. Br J Psychiatry 2004;185:55-62.

22. Van den Hout JH, Vlaeyen JW, Heuts PH, et al. Secondary prevention of work-related disability in nonspecific low back pain: does problem-solving therapy help? A randomized clinical trial. Clin J Pain 2003;19:87-96.

23. Bouman TK. Draaiboek 'Omgaan met ziektevrees', Probleem oplossen. [Treatment manual 'coping with health anxiety', Problem solving] Unpublished internal document. Groningen: University of Groningen, Department Clinical Psychology, 2000.

24. Kole-Snijders AM, Geurts SM, van den Hout JH, et al. Probleemoplossende vaardigheidstraining: een groepsen individueel programma. Handleiding voor therapeuten en werkboek voor deelnemers. [Problem solving skills training: a group and individual program. Manual for therapists and workbook for clients] Amsterdam: Boom test publishers, 2006.

25. Nezu AM. Cognitive appraisal of problem solving effectiveness: relation to depression and depressive symptoms. J Clin Psychol 1986;42:42-8.

26. Nezu AM, Nezu CM, Friedman SH, et al. A problem solving approach. Helping cancer patients to cope. Washington, DC: American Psychological Association, 1998.

27. Snaith RP. The Hospital Anxiety and Depression Scale. Health Qual Life Outcomes 2003;1:29.

28. Zigmond AS, Snaith RP. The Hospital Anxiety and Depression Scale. Acta Psychiatr Scand 1983;67:361-70.

29. Beck AT, Steer RA, Brown GK. Beck Depression Inventory second edition - Manual. San Antonio, TX: The Psychological Corporation, 1996.

30. Arnau RC, Meagher MW, Norris MP, et al. Psychometric evaluation of the Beck Depression Inventory-II with primary care medical patients. Health Psychol 2001;20:112-9.

31. Van der Does AJ. De Nederlandse versie van de Beck Depression Inventory - second edition (BDI-II-NL): Handleiding (in Dutch) [The Dutch Version of the Beck Depression Inventory - Second Edition (BDI-II-NL): A Manual]. San Antonio, TX: The Psychological Corporation, 2002.

32. Ware JE, Sherbourne CD. The MOS 36-item short-form health survey (SF-36). I. Conceptual framework and item selection. Med Care 1992;30:473-83.

33. Aaronson NK, Muller M, Cohen PD, et al. Translation, validation, and norming of the Dutch language version of the SF-36 Health Survey in community and chronic disease populations. J Clin Epidemiol 1998;51:1055-68.

34. Derogatis LR. The Brief Symptom Inventory. Baltimore, MD: Clinical Psychometric Research, 1975.

35. Arrindell WA, Ettema JH. SCL-90: Herziene handleiding bij een multidimensionele psychopathologie-indicator. [SCL-90: Revised manual for a multidimensional indicator of psychopathology]. Lisse, The Netherlands: Swets \& Zeitlinger, 2003. 
36. Karasek RA. Job Content Questionnaire and User's Guide. Los Angeles, University of Southern California: Department of Industrial and Systems Engineering, 1985.

37. Van Veldhoven $M$, Meijmen T. Het meten van psychosociale arbeidsbelasting met een vragenlijst: de Vragenlijst Beleving en Beoordeling van de Arbeid (VBBA) [Questionnaire on the Experience and Evaluation of Work]. Amsterdam: NIA, 1994.

38. Jacobson NS, Truax P. Clinical significance: a statistical approach to defining meaningful change in psychotherapy research. J Consult Clin Psychol 1991;59:12-9.

39. Lexis MA, Jansen NW, Stevens FC, et al. Experience of health complaints and help seeking behavior in employees screened for depressive complaints and risk of future sickness absence. J Occup Rehabil 2010;20(4):537-546.

40. Lerner D, Adler DA, Rogers WH, et al. Work performance of employees with depression: the impact of work stressors. Am J Health Promot 2010;24:205-13. 


\title{
6
}

The relationship between protocol adherence and the efficacy of a preventive intervention aimed at the prevention of long-term sickness absence and major depression

\author{
Monique A.S. Lexis $^{1}$ \\ Nicole W.H. Jansen ${ }^{1}$ \\ Marcus J.H. Huibers ${ }^{2}$ \\ Ludovic G.P.M. van Amelsvoort ${ }^{1}$ \\ Ate Berkouwer ${ }^{3}$ \\ Gladys Tjin A Ton ${ }^{3}$ \\ Piet A. van den Brandt ${ }^{1}$ \\ IJmert Kant ${ }^{1}$ \\ ${ }^{1}$ CAPHRI School for Public Health and Primary Care, Department of Epidemiology, \\ Maastricht University, Maastricht, The Netherlands \\ ${ }^{2}$ Department of Clinical Psychological Science, \\ Maastricht University, Maastricht, The Netherlands \\ ${ }^{3}$ Occupational Health Services Beter, Amsterdam, The Netherlands
}




\section{Abstract}

\section{Introduction}

In a randomized controlled trial (RCT), a new preventive intervention proved effective in prevention of long-term sickness absence and reduction of depressive complaints.

The aim of the study was to examine the relationship between protocol adherence and the efficacy of the preventive intervention.

\section{Methods}

Factors related to the intervention, such as the number of sessions, were assessed by registration forms at each session $(n=38)$. Depressive complaints were assessed by self-report using the Beck Depression Inventory (BDI-II) at baseline $(n=46), 6(n=30)$ and $12(n=29)$ months of follow-up. Sickness absence was assessed objectively for 12 months of follow-up.

\section{Results}

The number of sessions was associated with depressive complaints at 6 and 12 months of follow-up. The duration of the intervention and protocol execution were not associated with the outcomes. The reduction of depressive complaints, from baseline until 12 months of follow-up, was highest among employees who completed the intervention according to the basic part of the protocol (7 sessions), a reduction of 11.3 points on $\mathrm{BDI}-\mathrm{II}$.

\section{Conclusions}

The efficacy of the preventive intervention was associated with the number of sessions and was highest in those employees who completed the intervention according to the basic part of the protocol. 


\section{Introduction}

Depressive complaints are highly prevalent in the working population. ${ }^{1-3}$ When depressive complaints become severe and persistent, they may lead to major depression and long-term sickness absence. ${ }^{2,3}$ Even milder levels of depressive complaints have been found to be associated with long-term sickness absence. ${ }^{2,4}$ Conversely, sickness absence may also lead to depressive complaints. ${ }^{5,6}$ Long-term sickness absence, depressive complaints and depression have several consequences for employees, their families, workplaces and society. ${ }^{7}$ They constitute, for example, a source of marked distress and social impairment for the employee. Moreover, long-term sickness absence is associated with high costs for employers and society. Treatment and rehabilitation of employees on sick leave due to mental health problems still remains difficult. ${ }^{8,9}$ Therefore, a preventive approach was developed which aimed at prevention of both long-term sickness absence and major depression. A randomized controlled trial (RCT) was conducted to study the efficacy of a new preventive intervention. The target population of the RCT consisted of employees who had relatively mild health complaints and who were still at work. A screening instrument was used to identify those employees at high risk of future sickness absence and with mild to severe depressive complaints. The preventive intervention was based on principles of problem solving therapy (PST) and cognitive behavioral therapy (CBT). ${ }^{10}$ The intervention had substantial and statistically significant effects on the prevention of both long-term sickness absence and reduction of depressive complaints, in favor of the intervention group. The intervention was conducted according to a protocol specifically developed for the target population of the study, and participating psychologists were trained how to use the protocol. Despite the use of the protocol, some variation in the intervention could have occurred, which may have influenced the efficacy of the intervention. Variations between individual interventions were expected in the number of sessions, duration of the intervention and/or execution of the protocol by the psychologists who provided the intervention. For further improvement of the intervention, the aim of the present study was to examine the influence of variations in the intervention on the outcomes depressive complaints and sickness absence duration.

\section{Methods}

\section{Study design, procedure and participants}

This prospective study was conducted among employees working at a large banking company in the Netherlands and was based on results from a randomized controlled trial (RCT). The RCT aimed to examine the efficacy of early intervention on the prevention of future sickness absence and major depression. Details of the study are described elsewhere. ${ }^{10}$ In short, 211 employees were identified at high risk of future sickness absence and with mild to severe depressive complaints by a screening questionnaire. Employees who reported to be on sick leave, female employees who were pregnant or on pregnancy/maternity leave and employees who received treatment from a 
psychologist/psychiatrist at the time of completing the screening or baseline questionnaire were excluded. Finally, 139 employees were randomized into the intervention $(n=69)$ and the control group $(n=70)$. Employees randomized into the intervention group received a consultation with a company counselor, who informed them that they were identified at risk and who invited them to participate in the preventive intervention. The intervention was conducted by psychologists from a company consisting of a nationwide network of registered psychologists (Cenzo B.V.). The psychologists were selected based on their background and experience with cognitive behavioral therapy (CBT) and on the geographical position of their practice. The psychologists received two days of training before the study started and a one-day booster and evaluation session during the study.

\section{Preventive intervention}

The intervention consisted of a psychological treatment based on principles of problem solving therapy (PST) and cognitive behavioral therapy (CBT). The goal and content of the intervention are briefly summarized in Box 1 . The main goal of the intervention, which had an individual oriented approach, was to enhance problem solving skills, to reduce depressive complaints, to prevent long-term sickness absence and to stimulate personal well-being. The intervention was specifically developed for the study population by four experts in the field of psychology. A treatment protocol and workbooks for practical assignments were developed for the RCT based on two main (Dutch) sources. ${ }^{11-14}$ The focus of the protocol was on employees with relatively mild (health) complaints who were still at work. The intervention was preventive because of its timing, that is, treatment before sickness absence occurs, and because of its focus on mild complaints. Therefore, the intervention consisted for the greater part of PST. CBT and PST often consist of 10 to 12 sessions. This new intervention consisted of two parts, a basic part and a specific part. The basic part contained 7 sessions of 45 minutes each, based on the major steps of problem solving therapy. Through all sessions principles of cognitive behavioral therapy were applied. Seven sessions were recommended by the protocol, since the developers estimated that 7 sessions would be sufficient. However, it was unknown whether 7 sessions would be sufficient for all participants, so the intervention could be extended with a maximum of five additional sessions. The protocol recommended a total duration of the intervention of four months. The $7^{\text {th }}$ session consisted of an evaluation session in which the psychologist and the employee decided in consultation to end treatment in case the participant had recovered, or to continue with the specific part of the protocol. Each session had a fixed structure: review of the last session, discussion of the homework assignment, introduction of a new problem solving skill, training of the new skill, a new homework assignment and preview of the next session. The last treatment session included prevention of relapse and evaluation of the intervention.

\section{Aims of the sessions}

Sessions 1 to 6 focused on the rationale and introduction of problem solving skills. Participants learned to identify problems ('what are the most important problems and why are they a problem?'), to formulate aims ('what do I want to achieve and why?'), to think of solutions ('what are possible 
solutions?'), to make decisions ('what solution fits the aim?') and finally to implement the solutions ('how do I put the solution into practice?'). During all sessions principles of CBT were applied, such as identification of emotional (for example anxiety, anger, and restlessness) and behavioral (being more quiet, complaining, eating too much) signals belonging to stress and barriers in daily living, and conversion of negative into positive thoughts.

The $7^{\text {th }}$ session consisted of an interim evaluation session whereupon three options for continuation could be made. The first option was to continue with the specific part of the protocol. If problems were still too serious to cover within five additional sessions, the second option was to refer the employee to another discipline. The third option was to end treatment in case the participant had recovered or was capable of applying the treatment principles successfully by him/her self. For sessions 8 to 11 employees could choose modules meeting their personal problems. A minimum of two sessions for each module was desirable to promote the employee to practice (doing homework, reflection and looking up information) between two sessions. Session 12, or the last session when the intervention was finished earlier, contained the final evaluation of the content and process of the intervention.

\section{Box 1 Content of the preventive intervention}

\section{GOAL OF THE PREVENTIVE INTERVENTION:}

To enhance problem solving skills in order to reduce depressive complaints STEPS IN THE INTERVENTION:

Basic part: maximum of 7 sessions

- Explanation rationale and introduction problem solving skills

- Identification of problems

- Formulation of aims

- Thinking of solutions

- Making decisions

- Implementation of solutions

- Evaluation and decision to end or continue the intervention

Specific part: maximum of 5 additional sessions to the basic part

- Individual choice of modules (such as cognitive restructuring, training of social skills)

- Evaluation and closing

\section{Measurements}

\section{Factors related to the intervention}

Three main factors were identified that could have led to variation between individual interventions. These factors were the number of sessions, the duration of the intervention and protocol execution 
by the psychologists. Information on these factors was assessed using registration forms completed by the psychologists after each session. Of the 46 employees who participated in the intervention 38 returned their workbooks including the registration forms. Their information could be included in the analyses.

\section{Number of sessions}

For each participant, the number of sessions received was counted. Based on the number of sessions, three groups were defined: 1) participants who completed the intervention according to the basic part of the protocol, including 7 sessions $(n=10), 2$ ) participants who completed the basic part of the intervention and who received one to five additional sessions of the specific part of the intervention $(n=19)$, and 3) participants who did not complete the intervention according to protocol ( $<7$ sessions $(n=17))$. For only 9 of these 17 participants workbooks were available. The latter group was not defined by the protocol, but after receiving the workbooks it became clear that a considerable number of employees received less than the recommended 7 sessions.

\section{Duration of intervention}

For each participant, the total duration of the intervention period was counted in days. A total duration of four months was recommended by the protocol, but the duration varied substantially between employees.

\section{Protocol execution}

To assess adherence to the treatment protocol, psychologists used standardized registration forms on which the steps addressed in each session and a short summary of the content of the session were registered. For each session it was determined whether the intervention was performed according to the protocol. Psychologists ticked off the steps carried out in each session and these were compared with the steps required by the protocol. The match was defined as the percentage of adherence to the protocol. For the total intervention the mean adherence to the protocol was calculated.

\section{Primary outcomes}

\section{Depressive complaints}

To assess the presence and severity of depressive complaints the Dutch version of the widely used Beck Depression Inventory-II (BDI-II) was used. ${ }^{15}$ Scores on the BDI-II can range from 0 to 63, with higher scores indicating more severe depressive symptoms. ${ }^{16}$ The BDI-II was included in the baseline questionnaire and in the 6 and 12 months follow-up questionnaires. At 6 months of follow-up, 30 employees responded and at 12 months of follow-up 29 employees responded to the BDI-II. 


\section{Sickness absence duration}

Sickness absence duration was measured objectively (in calendar days) through record linkage on an individual level with the company registers on certified sickness absence, from two months after employees received the screening questionnaire until 12 months of follow-up. This is in line with the development of the Balansmeter. ${ }^{17}$ For all 46 employees who started the intervention sickness absence data were available.

\section{Secondary outcomes}

Data on secondary outcome measures were gathered by means of self-administered questionnaires. Besides the BDI-II, the presence and severity of depressive complaints was assessed using the depression scale of the Hospital Anxiety and Depression Scale (HAD Scale), in the screening questionnaire and at 6 and 12 months of follow-up. The HAD Scale is a 14 item self-report questionnaire, that was originally developed to indicate the presence and severity of both anxiety (HAD-A) and depression (HAD-D) separately (range 0-21). ${ }^{18,19}$ The Brief Symptom Inventory (BSI), ${ }^{20}$ a short version of the Symptom Checklist 90 (SCL-90), ${ }^{21}$ was used to assess general psychological distress, with higher scores indicating a higher degree of symptoms (range 0-212). Satisfaction with the intervention was assessed with an evaluation questionnaire (range 0-10), which participants received after they completed the 12-month follow-up questionnaire.

\section{Demographic factors}

Employees provided information on gender, age and educational level (low, medium or high) through self-report in the screening questionnaire.

\section{Statistical analyses}

Univariate linear regression analyses were performed to study the associations between the number of sessions, the duration of the intervention and protocol execution by the psychologists and the outcome depressive complaints at 6 and 12 months of follow-up. Poisson regression analyses were performed to study the association between the three factors and the outcome sickness absence duration. Due to too low power in the subgroups, multivariate regression analyses could not be performed. Graphs were used to further explore the associations. 


\section{Results}

\section{Participants}

In total, 69 employees identified at high risk of future sickness absence and with depressive complaints were randomly allocated to the intervention group of the RCT. 17 of the 69 employees instantaneously refused to participate in the intervention. Of the 49 who agreed to receive treatment, 3 employees did still not start the intervention and 8 employees received one treatment session only. Reasons for these 8 employees for not continuing the intervention were: not willing to participate $(n=2)$, not seeing the usefulness of treatment $(n=4)$, leaving the company $(n=1)$, referral to different kind of treatment $(n=1) .46$ employees participated in the intervention and of them 38 returned their workbooks including the session registration forms. Table 1 presents the descriptive characteristics of the employees who participated in the intervention. Table 2 presents characteristics of the intervention.

Table 1 Descriptive characteristics of employees randomized into the intervention group who participated in the preventive intervention $(\mathrm{n}=46)$

\begin{tabular}{|c|c|}
\hline Variables & $\begin{array}{l}\text { Participants who received } \\
\text { at least one treatment } \\
\text { session }\end{array}$ \\
\hline Gender, male; N $(\%)^{*}$ & $29(63.0)$ \\
\hline Age (18-65); mean (SD) & $47.59(8.84)$ \\
\hline \multicolumn{2}{|l|}{ Highest educational level; $\mathrm{N}(\%) \dagger$} \\
\hline Low & $4(9.8)$ \\
\hline Medium & $31(75.6)$ \\
\hline High & $6(14.6) 9$ \\
\hline \multicolumn{2}{|c|}{ Depressive complaints (BDI-II), (0-63) ‡; Mean (SD), based on available questionnaires } \\
\hline Baseline (T0), (n=46) & $18.15(9.42)$ \\
\hline After 6 months (T1), $(\mathrm{n}=30)$ & $14.00(11.50)$ \\
\hline After 12 months (T2), (n=29) & $12.31(10.01)$ \\
\hline \multicolumn{2}{|c|}{ Sickness absence duration in days during follow-up; mean (SD) } \\
\hline $0-12 * *$ & $24.29(34.18)$ \\
\hline $0-18 \S$ & $33.05(51.18)$ \\
\hline
\end{tabular}

* Dichotomous variable (no / yes) with $\mathrm{N}(\%)$ indicating 'yes'; † categorical variable with $\mathrm{N}(\%)$ indicating 'yes'; ‡ scale range;

** assessed from baseline until 12 months of follow-up; § assessed from baseline until 18 months of follow-up; ๆ numbers do not add up to 46 due to missing values. 
Table 2 Characteristics of the intervention of employees who participated in the intervention and who returned their workbooks ( $\mathrm{n}=38$ )

\begin{tabular}{ll}
\hline Variables & Mean (SD) \\
\hline Total duration of intervention (days) & $130(63.85)$ \\
Protocol execution by the psychologists; \% of steps conducted according to protocol & $95.0(12.63)$ \\
Number of sessions & $7.5(2.53)$ \\
\hline
\end{tabular}

Table 3 Univariate associations between duration of the intervention, protocol execution and number of sessions and the outcomes depressive complaints and sickness absence duration

\begin{tabular}{|c|c|c|c|c|c|c|}
\hline \multirow[t]{2}{*}{ Variables } & \multicolumn{2}{|c|}{$\begin{array}{l}\text { Score on BDI-II at } 6 \\
\text { months of follow-up } \\
(n=30)\end{array}$} & \multicolumn{2}{|c|}{$\begin{array}{l}\text { Score on BDI-II at } 12 \\
\text { months of follow-up } \\
(n=29)\end{array}$} & \multicolumn{2}{|c|}{$\begin{array}{l}\text { Sickness absence } \\
\text { duration for } 12 \text { months of } \\
\text { follow-up }(n=46)\end{array}$} \\
\hline & B $(95 \% \mathrm{Cl})$ & $p$-value & B (95\% Cl) & $p$-value & B $(95 \% \mathrm{Cl})$ & $\mathrm{p}$-value \\
\hline $\begin{array}{l}\text { Duration of the } \\
\text { intervention }\end{array}$ & $\begin{array}{l}-0.03 \\
(-0.11-0.04)\end{array}$ & 0.366 & $\begin{array}{l}-0.03 \\
(-0.10-0.04)\end{array}$ & 0.429 & $\begin{array}{l}-0.00 \\
(-0.01-0.01)\end{array}$ & 0.719 \\
\hline Protocol execution & $\begin{array}{l}0.03 \\
(-0.27-0.32)\end{array}$ & 0.851 & $\begin{array}{l}0.05 \\
(-0.37-0.48)\end{array}$ & 0.802 & $\begin{array}{l}0.01 \\
(-0.03-0.04)\end{array}$ & 0.705 \\
\hline \multicolumn{7}{|l|}{ Number of sessions } \\
\hline$<7$ sessions vs. 7 sessions & $\begin{array}{l}5.17 \\
(-7.08-17.42)\end{array}$ & 0.369 & $\begin{array}{l}8.95 \\
(-4.13-22.03)\end{array}$ & 0.160 & $\begin{array}{l}0.80 \\
(-0.63-2.23)\end{array}$ & 0.275 \\
\hline$>7$ sessions vs. 7 sessions & $\begin{array}{l}11.81 \\
(3.25-20.37)\end{array}$ & 0.010 & $\begin{array}{l}9.21 \\
(1.55-16.88)\end{array}$ & 0.021 & $\begin{array}{l}0.87 \\
(-0.24-1.98)\end{array}$ & 0.126 \\
\hline
\end{tabular}

Table 3 presents univariate associations between the duration of the intervention, protocol execution by the psychologists, the number of sessions and the outcomes depressive complaints and sickness absence duration. Significant associations were only found in depressive complaints at 6 and 12 months of follow-up for employees who received more than 7 sessions compared with employees who completed the intervention according to the basic part of the protocol (7 sessions). The relationship between number of sessions and sickness absence duration showed no significant associations.

The relationship between the number of sessions was then further explored. In each of the three groups (<7, 7 or $>7$ sessions), BDI-II scores decreased between baseline, 6 and 12 months of follow-up (figure 1). Scores on BDI-II decreased mostly in the group of employees who completed the intervention according to the basic part of the protocol, a reduction of 11.3 points, compared with 3.3 points among those who did not complete the intervention ( $<7$ sessions) and 2.5 points in employees who received more than the recommended 7 sessions. The differences between the groups appeared to be of high clinical relevance. However, no significant differences in depressive complaints were found between the groups at 6 or 12 months of follow-up. The group of employees who completed the intervention according to the basic part of the protocol also showed a lower 
mean sickness absence duration compared with the other two groups at 12 months of follow-up (figure 2), but these differences were also not significant. Figures 3 and 4 present the results for the secondary outcomes depressive complaints assessed by HAD-D and psychological distress assessed by BSI. For both HAD-D and BSI, results were largely similar to those found for BDI-II. In general, scores on HAD-D and BSI decreased the most during follow-up among employees who completed the intervention according to the basic part of the protocol, but the differences between the groups, although clinically relevant, failed to reach statistical significance.

In the evaluation questionnaire, employees who completed the intervention according to the basic part of the protocol reported a mean score of satisfaction of 7.4 and they all reported that the intervention yielded what they expected. Non-completers reported a score of 6.1 and completers with additional sessions gave a mean score of 7.0. Of these two groups, 7 of the 28 employees reported that the intervention did not yield what they expected from it.

Figure 1 Mean total scores on BDI-II at baseline, 6 and 12 months of follow-up for employees who received $<7,7$ or $>7$ sessions

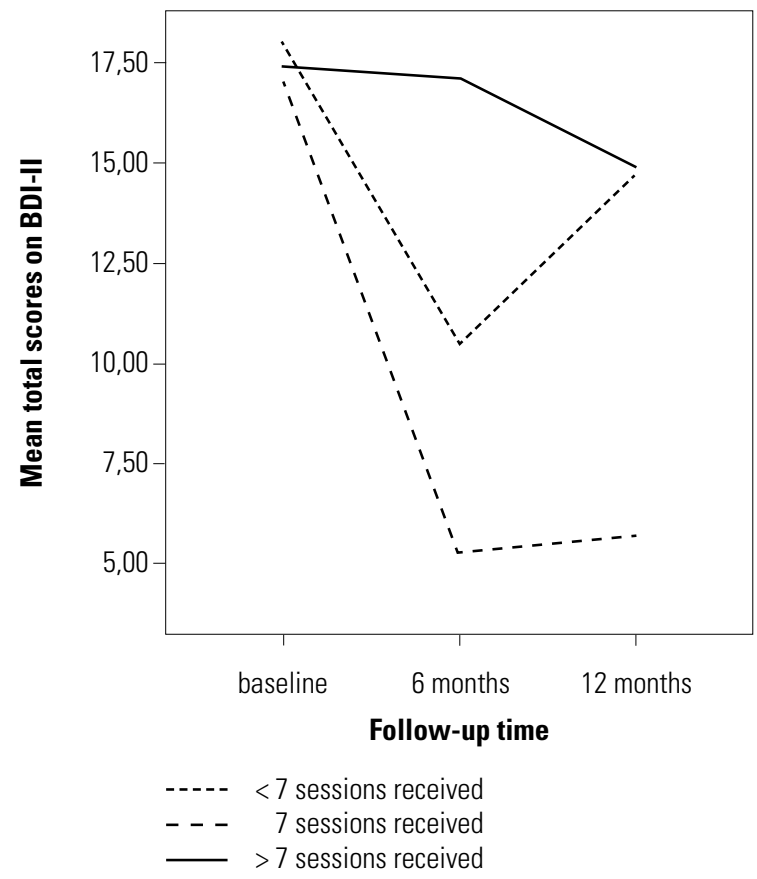

\begin{tabular}{lccc}
\hline & \multicolumn{3}{c}{ Number of sessions received } \\
\cline { 2 - 4 } $\begin{array}{l}\text { Score on } \\
\text { BDI-II; }\end{array}$ & $<7$ & 7 & $>7$ \\
mean (SD) & & & \\
\hline Baseline & 18.00 & 17.00 & 17.42 \\
& $(10.71)$ & $(10.15)$ & $(6.97)$ \\
\hline 6 months & 10.50 & 5.33 & 17.14 \\
& $(12.82)$ & $(4.13)$ & $(9.49)$ \\
\hline 12 months & 14.67 & 5.71 & 14.93 \\
& $(15.20)$ & $(4.07)$ & $(9.16)$ \\
\hline
\end{tabular}


Figure 2 Mean total sickness absence duration for employees who received $<7,7$ and $>7$ sessions for 12 months of follow-up

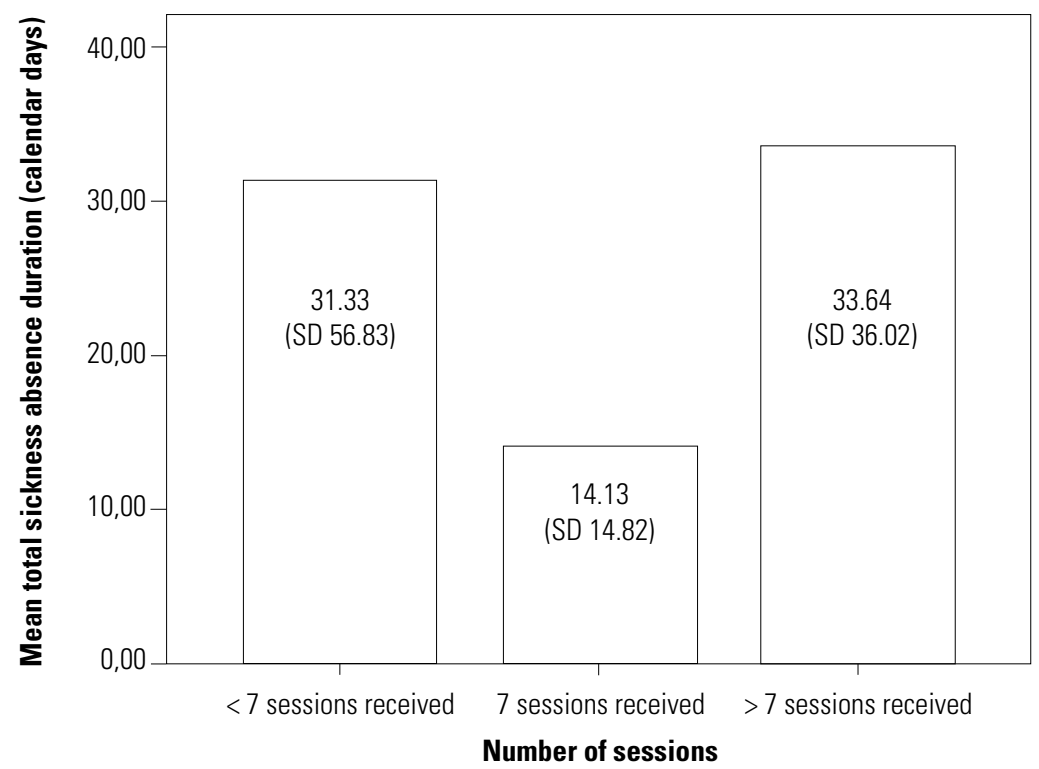

Figure 3 Mean total scores on HAD-D at baseline, 6 and 12 months of follow-up for employees who received $<7,7$ and $>$ 7 sessions

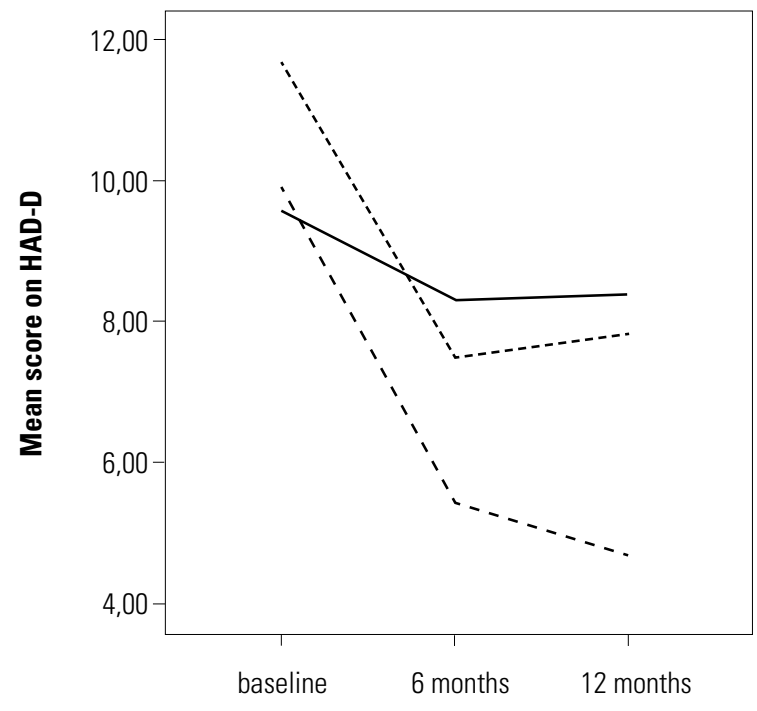

\begin{tabular}{lccc}
\hline & \multicolumn{3}{c}{ Number of sessions received } \\
\cline { 2 - 4 } Score on & $<7$ & 7 & $>7$ \\
HAD-D; & & & \\
mean (SD) & & & \\
\hline Screening & 11.67 & 9.90 & 9.58 \\
& $(2.78)$ & $(2.18)$ & $(1.68)$ \\
\hline 6 months & 7.50 & 5.43 & 8.29 \\
& $(5.21)$ & $(3.82)$ & $(3.69)$ \\
\hline 12 months & 7.83 & 4.71 & 8.83 \\
& $(5.67)$ & $(3.82)$ & $(4.23)$ \\
\hline
\end{tabular}

\section{Follow-up time}

-.-- $<7$ sessions received

- - $\quad 7$ sessions received

$\longrightarrow 7$ sessions received 
Figure 4 Mean total scores on BSI at baseline, 6 and 12 months of follow-up for employees who received $<7,7$ or $>7$ sessions

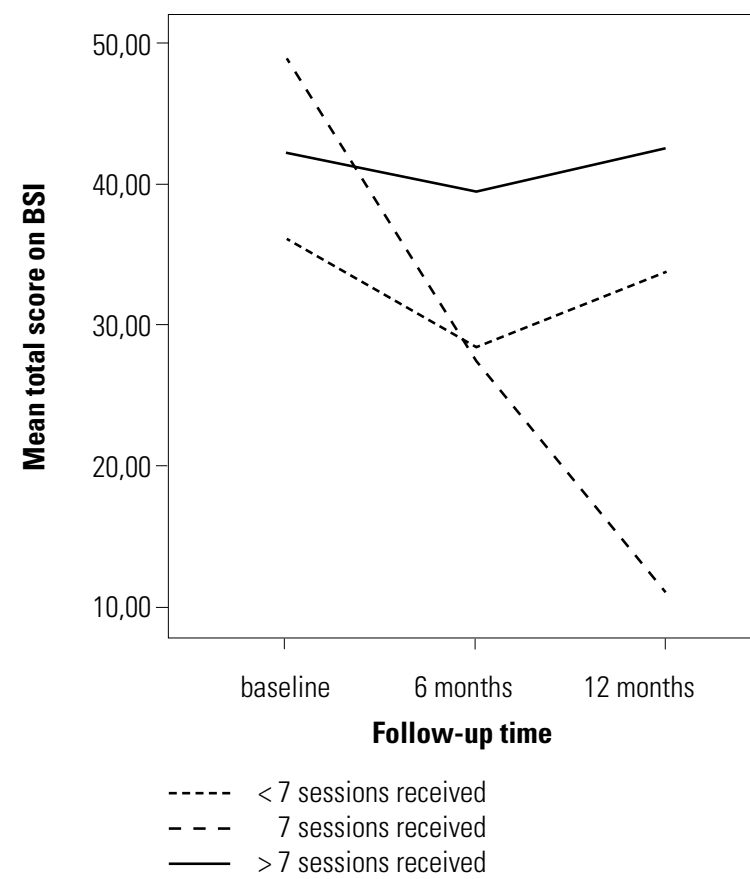

\begin{tabular}{lccc}
\hline & \multicolumn{3}{c}{ Number of sessions received } \\
\cline { 2 - 4 } $\begin{array}{l}\text { Score on } \\
\text { BSI; }\end{array}$ & $<7$ & 7 & $>7$ \\
mean (SD) & & & \\
\hline Baseline & 36.00 & 48.80 & 42.11 \\
& $(31.17)$ & $(32.45)$ & $(21.60)$ \\
\hline 6 months & 28.33 & 27.43 & 39.25 \\
& $(42.40)$ & $(27.30)$ & $(32.64)$ \\
\hline 12 months & 33.67 & 11.14 & 42.36 \\
& $(52.26)$ & $(10.29)$ & $(30.26)$ \\
\hline
\end{tabular}

\section{Discussion}

\section{Main findings and interpretation of results}

Recently, a randomized controlled trial (RCT) was conducted in which a new preventive intervention proved to be effective in the prevention of long-term sickness absence and reduction of depressive complaints. ${ }^{10}$ The intervention was conducted according to a protocol which was specifically developed for the target population of the study. The target population consisted of employees identified at high risk of future sickness absence and with mild to severe depressive complaints. Participating psychologists were trained how to use the protocol. However, some variation in the performance of the intervention could have occurred, which may have had an influence on the overall efficacy of the intervention. The aim of the present study was to examine the influence of possible variation in performance of the intervention on the outcomes depressive complaints and sickness absence duration.

The univariate regression analyses showed a statistically significant association between the number of sessions and depressive complaints at 6 and 12 months of follow-up. Figures 1 and 2 showed that the number of sessions $(<7,7$ or $>7$ ) had a substantial influence on the outcomes. Regarding depressive complaints, employees who received the basic part of 7 sessions clearly showed the 
best results after 6 and 12 months of follow-up. Employees who received more than 7 sessions had the least improvement in scores on BDI-II. Scores on BDI-II of employees who received less than 7 sessions decreased between baseline and 6 months of follow-up, but increased between 6 and 12 months of follow-up. At 12 months of follow-up, they had improved only a little more than the group of employees who received more than 7 sessions. Similar patterns were found for depressive complaints assessed by HAD-D, and for psychological distress, assessed by BSI. These results support the robustness of the results found for BDI-II, despite the small subgroups and nonsignificant findings. For sickness absence duration similar results were found as well, with employees who completed the intervention according to the basic part of the protocol having the lowest number of sickness absence days at 12 months of follow-up. However, no statistically significant differences were found between the three groups on each of the outcomes, probably due to too low power. The three groups were compared on many characteristics, such as gender and age, but no statistically significant or clinically relevant differences were found between the three groups. Moreover, the three groups were compared on work characteristics, such as job demands and social support, again no statistically significant differences were found between the groups (specific data not shown). Nevertheless, with regard to the difference in total sickness absence days between the employees who received the basic part of 7 sessions and the other two groups, the difference was approximately 15 days, which is quite a big difference considering the total mean sickness absence duration of 24.29 (SD 34.18) sickness absence days in the total intervention group.

Of the 69 employees who were randomized into the intervention group of the RCT, 46 (66.7\%) actually participated in the intervention. 38 of these 46 employees returned their workbooks (55.1\%). This compliance rate was considered acceptable taking into account the preventive approach in which employees with relatively mild (health) complaints were selected. However, only 30 and 29 employees completed the BDI-II at 6 and 12 months of follow-up respectively, which may indicate selective withdrawal of employees during follow-up. BDI-Il scores of completers and non-completers did not differ at baseline, but sufficient information on other psychological factors was not available to identify whether withdrawal was selective.

The regression analyses showed no statistically significant associations between duration of the intervention and protocol execution and depressive complaints. The duration of the intervention was expected to be associated with the outcomes. The protocol recommended a-four-month duration of the intervention. Although the mean duration of all interventions was approximately 4 months (130 (SD 63.85) days), a considerable variation was found in the duration of intervention periods. Many intervention periods took longer than four months, but shorter periods were reported as well. The main reason for a longer duration was that employees were not able to receive a session each week, due to for example too busy work schedules or holidays. Protocol execution was not found to be associated with the outcomes as well. Despite the use of a protocol, psychological treatment leaves some more space for variations in performance than for example a pharmacological treatment. However, protocol execution as reported by the psychologists was quite high in our study and the variation in protocol execution quite low (mean 95.0\% SD (12.63)). 


\section{Possible explanations for the influence of number of sessions on the outcomes}

As determined in the protocol, 7 sessions were recommended. Nevertheless, since the target population of the study had never been studied before, it was not certain if this number of sessions would be sufficient for every participant. Therefore, psychologists were asked to decide together with the employee when to end treatment. This way of decision making was rather subjective and may have influenced the results.

Employees who received more than 7 sessions showed the least decrease in depressive complaints between baseline and 6 months of follow-up. Finally, at 12 months of follow-up they had the highest score on BDI-II. These results might indicate that the complaints and/or problems of these participants were too complex to solve with this type of preventive intervention. This seems to be a likely explanation, since the treatment protocol prescribed that not being recovered at the $7^{\text {th }}$ session was a valid reason to continue treatment.

Of the 19 participants in this group, 9 participants received 8 sessions, only one more than the recommended 7 sessions. Explanations for these results might be that the specific part of the intervention did not meet their needs, or they might not have believed the specific part of the intervention would be effective in solving their problems within the remaining sessions.

Employees who did not complete the intervention according to protocol, but who received less than 7 sessions, might have ended treatment too soon and they might have had more benefit if they completed the intervention according to protocol. A reason for not completing the intervention according to protocol might be explained by the preventive nature of the study. In this study, employees were identified with relatively mild health complaints by an objective screening instrument. A previous study by Lexis et al. among the same study population showed that of the 211 employees identified at high risk of future sickness absence and depressive complaints, only 140 (69\%) reported to experience some kind of health complaints at the moment of completing the screening questionnaire. ${ }^{22}$ Of the employees who reported not to have already sought help for the complaints, only $50 \%$ reported to have the intention to seek help. These findings might suggest that employees who did not complete the intervention according to protocol were employees who did not experience health complaints or did not have the intention to accept treatment for the health complaints at the moment of participation in the intervention. Hence, the nature of the intervention might have fitted best to those employees who completed the intervention according to protocol. It is widely reported in the literature that those persons who show early response to treatment improve the most. ${ }^{23,24}$ Figure 1 showed that the higher the decrease in depressive complaints between baseline and 6 months of follow-up, the lower the final score on BDI-II at 12 months. This might suggest the effect of so-called 'sudden gains', a sudden and considerable drop in symptoms ratings in between consecutive sessions. Several studies on sudden gains in CBT for depression found that sudden improvements in symptomatology in the first sessions of treatment have significantly larger decreases in depressive symptoms over the course of treatment and are more likely to be treatment responders. ${ }^{25,26}$ This might partly explain why scores on BDI-II of employees who received the basic part of 7 sessions decreased the most between baseline and 6 months and decreased even 
more between 6 and 12 months of follow-up. However, as depressive complaints were assessed at baseline, 6 and 12 months of follow-up, no information was available on the exact course of depressive complaints over time.

Another important factor affecting the effect of the psychological intervention might have been the personal relationship between the psychologist and the employee. Differences in attitude or treatment style might have had an influence on completion of the intervention as well.

In general, psychologists are trained to work in a demand-driven way. When using a preventive strategy, a demand-driven approach was not considered appropriate. In the two-day training psychologists were pointed at this and were taught to take more initiative themselves. Employees who ended participation after one treatment session might have been influenced by the procedure used by the psychologist. However, the decision to end treatment by the employee might have been influenced by the satisfaction with treatment as well. Some employees reported that they did not see the usefulness of this type of intervention or they did not want to participate in a psychological treatment.

Psychologists with experience in delivering CBT were selected to participate in the RCT. They received a two-day training prior to the start of the RCT to learn how to use the new protocol and they received a one-day booster session during the intervention period. The training might have been too brief or some psychologists might have lacked the necessary experience to deliver the intervention in the most optimal way for all participants.

\section{Methodological considerations}

Strengths of the study included the study design (RCT), the objective assessment of sickness absence and the long period of follow-up of sickness absence and depressive complaints. A limitation of the study was the low power due to small subgroups, and for explanation of the differences found between the subgroups, more information on baseline characteristics would have been desirable. Another limitation of the study was that although we were able to assess the quantity of the steps in the intervention, we were not able to assess the quality of the intervention delivered. Registration forms instead of audiovisual recordings were used to assess the intervention, which might have led to socially desirable responses of the psychologists. Some differences between psychologists could be observed using the self-administered registration forms, such as the number of participants treated by each psychologist. This mainly depended on the geographical position of the psychologists' practices. Additional information on how well the intervention was performed might have elucidated the results. The efficacy of the preventive intervention was evaluated among employees in an office work environment. Therefore, this study is not fully representative for the general working population. It is believed that the intervention may be suitable for similar companies in the occupational health care setting, because of the occurrence of comparable (health) complaints and problems.

\section{Implications and future research}

The preventive intervention proved effective in the prevention of long-term sickness absence and reduction of depressive complaints among high risk employees, which makes the preventive 
intervention a valuable instrument for implementation in occupational health care. For implementation some issues should be considered. For the RCT, company counselors and psychologists were trained to conduct the intervention. For successful implementation of the preventive intervention in occupational health care, sufficient psychologists with experience in CBT/PST should be available, as well as supporting staff to conduct the screening procedure and company counselors for the guidance of selected employees to the intervention. All participating professionals should be trained.

The per-protocol analyses of the RCT showed that receiving only a small number of sessions was already effective. ${ }^{10}$ This study showed that those employees who completed the intervention according to the basic part of the protocol had the best results. However, it should be noted that our findings do not imply that receiving 7 sessions in itself leads to better outcomes. Therefore, future research is needed to explore whether there is a dose-response relationship between the number of sessions and the outcomes, and to determine the optimal number of sessions for each individual participant. A more tailored or stepped-care approach might be even more effective and such an approach might better meet the needs of individual employees.

\section{Acknowledgements}

This study was financially supported by the Netherlands Organization for Health Research and Development (ZonMw), grant no. 62200024, by CAPHRI School for Public Health and Primary Care, Maastricht, the Netherlands and by the Occupational Health Services Beter (ABN AMRO Arbo Services), Amsterdam, the Netherlands.

The authors thank A. Arntz and M. Huibers (Maastricht University), T. Bouman (University Medical Center Groningen) and A. van den Hout (Academic Community Health Centre Maastricht), experts in the field of psychology, for development of the protocol and workbooks for the preventive intervention and training of the participating psychologists. The authors also thank D. de Bruyn, S. Dijkstra, H. Kalter, W. ten Haaf and P. Batting, company counselors from Occupational Health Services Beter, for conducting the consultations. The authors also thank I. Pos, director of Cenzo B.V., for coordination of execution of the intervention, and J. Biemans, S. Decoz, J. Haasnoot, A. Janssen, A. Kuipers, G. Langenhuizen, M. Ooms, G. Oppewal, R. Verhoog and M. Udenhout, psychologists from Cenzo B.V., for providing the psychological intervention. The authors thank J. Slangen and P. Brouns (Maastricht University), B. Couwenberg and M. Binsbergen (Occupational Health Services Beter), for their contribution to data management. 


\section{References}

1. Andrea H, Bultmann U, Beurskens AJ, Swaen GM, van Schayck CP, Kant IJ. Anxiety and depression in the working population using the HAD Scale--psychometrics, prevalence and relationships with psychosocial work characteristics. Soc Psychiatry Psychiatr Epidemiol. 2004;39:637-46.

2. Lexis MA, Jansen NW, van Amelsvoort LG, van den Brandt PA, Kant I. Depressive complaints as a predictor of sickness absence among the working population. J Occup Environ Med. 2009;51:887-95.

3. Laitinen-Krispijn S, Bijl RV. Mental disorders and employee sickness absence: the NEMESIS study. Netherlands Mental Health Survey and Incidence Study. Soc Psychiatry Psychiatr Epidemiol. 2000;35:71-7.

4. Cuijpers P, Smit F. Subthreshold depression as a risk indicator for major depressive disorder: a systematic review of prospective studies. Acta Psychiatr Scand. 2004;109:325-31.

5. Melchior M, Ferrie JE, Alexanderson K, Goldberg M, Kivimaki M, Singh-Manoux A, et al. Using sickness absence records to predict future depression in a working population: prospective findings from the GAZEL cohort. Am J Public Health. 2009;99:1417-22.

6. Vahtera J, Westerlund H, Ferrie JE, Head J, Melchior M, Singh-Manoux A, et al. All-cause and diagnosisspecific sickness absence as a predictor of sustained sub-optimal health: a 14-year follow-up in the GAZEL cohort. J Epidemiol Community Health. 2009;64:311-7.

7. Marmot M, Feeney A, Shipley M, North F, Syme SL. Sickness absence as a measure of health status and functioning: from the UK Whitehall II study. J Epidemiol Community Health. 1995;49:124-30.

8. Brouwers EP, Tiemens BG, Terluin B, Verhaak PF. Effectiveness of an intervention to reduce sickness absence in patients with emotional distress or minor mental disorders: a randomized controlled effectiveness trial. Gen Hosp Psychiatry. 2006;28:223-9.

9. Huibers MJ, Beurskens AJ, Van Schayck CP, Bazelmans E, Metsemakers JF, Knottnerus JA, et al. Efficacy of cognitive-behavioural therapy by general practitioners for unexplained fatigue among employees: Randomised controlled trial. Br J Psychiatry. 2004;184:240-6.

10. Lexis M, Jansen N, Huibers M, Van Amelsvoort L, Berkouwer A, Tjin A Ton G, van den Brandt PA, Kant IJ, et al. Prevention of long-term sickness absence and major depression in high-risk employees: a randomised controlled trial. Occup Environ Med, in press.

11. Bouman TK. Draaiboek 'Omgaan met ziektevrees', Probleem oplossen. [Treatment manual 'coping with health anxiety', Problem solving] Unpublished internal document. Groningen: University of Groningen, Department Clinical Psychology, 2000.

12. Kole-Snijders AM, Geurts SM, van den Hout JH, et al. Probleemoplossende vaardigheidstraining: een groepsen individueel programma. Handleiding voor therapeuten en werkboek voor deelnemers. [Problem solving skills training: a group and individual program. Manual for therapists and workbook for clients] Amsterdam: Boom test publishers, 2006.

13. Nezu AM. Cognitive appraisal of problem solving effectiveness: relation to depression and depressive symptoms. J Clin Psychol. 1986;42:42-8.

14. Nezu AM, Nezu CM, Friedman SH, Faddis S, Houts PS. A problem solving approach. Helping cancer patients to cope. Washington DC: American Psychological Association; 1998. 
15. Beck AT, Steer RA, Brown GK. Beck Depression Inventory second edition - Manual. San Antonio TX:The psychological Corporation, 1996.

16. Arnau RC, Meagher MW, Norris MP, Bramson R. Psychometric evaluation of the Beck Depression Inventory-II with primary care medical patients. Health Psychol. 2001;20:112-9.

17. Kant IJ, Jansen NW, van Amelsvoort LG, Swaen GM, van Leusden R, Berkouwer A. Screening questionnaire Balansmeter proved successful in predicting future long-term sickness absence in office workers. J Clin Epidemiol. 2009;62:408-14.

18. Snaith RP. The Hospital Anxiety And Depression Scale. Health Qual Life Outcomes. 2003;1:29.

19. Zigmond AS, Snaith RP. The hospital anxiety and depression scale. Acta psychiatr Scand. 1983;67:361-70.

20. Derogatis LR. The Brief Symptom Inventory. Baltimore, MD.: Clinical Psychometric Research. 1975.

21. Arrindell WA, Ettema JHM. SCL-90: Herziene handleiding bij een multidimensionele psychopathologieindicator. [SCL-90: Revised manual for a multidimensional indicator of psychopathology]. Lisse, The Netherlands: Swets \& Zeitlinger;2003.

22. Lexis M, Jansen N, Stevens F, van Amelsvoort L, Kant I. Experience of health complaints and help seeking behavior in employees screened for depressive complaints and risk of future sickness absence. J Occup Rehabil 2010;20(4):537-546.

23. Beck AT, Ward CH, Mendelson M, Mock J, Erbaugh J. An inventory for measuring depression. Arch Gen Psychiatry 1961;4:561-71.

24. Blackburn, I.M., Bishop, S. Changes in cognition with pharmacotherapy and cognitive therapy. Br J Psychiatry 1983;143:609-17.

25. Kelly MA, Roberts JE, Ciesla JA. Sudden gains in cognitive behavioral treatment for depression: when do they occur and do they matter? Behav Res Ther. 2005;43:703-14.

26. Busch AM, Kanter JW, Landes SJ. Sudden gains and outcome: a broader temporal analysis of cognitive therapy for depression. Behav Ther 2006;37:61-68. 
General discussion 

The general aim of this thesis was to examine if a preventive strategy aimed at employees who are at high risk of future sickness absence and who have mild to severe depressive complaints is effective in the prevention of long-term sickness absence and major depression. In this general discussion, the main findings of the study are discussed and methodological considerations are described. Furthermore, considerations regarding implementation of the preventive strategy, implications for different stakeholders, suggestions for optimalization of the preventive strategy, recommendations for future research and the overall conclusion are presented.

\section{Main findings}

The basic assumption of the general aim of the study was that depressive complaints are associated with (future) sickness absence. This relationship was studied in the first chapter. The results of this study show that even mild levels of depressive complaints are associated with (future) sickness absence. Moreover, more severe depressive complaints are associated with longer sickness absence duration and shorter time to onset of the first sickness absence spell. These findings confirmed and further specified the relationship between relatively mild depressive complaints and sickness absence.

A prerequisite for the use of a preventive strategy is the ability to identify employees at high risk of future sickness absence and with mild to severe depressive complaints. The screening instrument Balansmeter proved effective in predicting future long-term sickness absence in both male and female employees with mild to severe depressive complaints. Employees with relatively mild (health) complaints were selected by the screening instrument, which is characteristic of the preventive approach. The selection of relatively healthy employees resulted in a substantial part of the employees identified at high risk by the screening instrument who reported not to experience health complaints. Since experience of health complaints is known to be the most important prerequisite for help seeking behavior, this may have been an important reason for employees to refuse participation in the preventive intervention. Although certain measures to optimize participation in the RCT were taken, such as a consultation with a company counselor before the start of the study, there were still employees who did not participate in the intervention because they indicated not to experience health complaints.

The preventive intervention proved effective in the prevention of long-term sickness absence and reduction of depressive complaints in high-risk employees. Substantial differences in total sickness absence duration and depressive complaints were found between the intervention and control group of the RCT, in favor of the intervention group. Besides the effects on sickness absence and depressive complaints, the preventive intervention also showed amongst others significantly lower psychological distress in the intervention group compared with the control group. A new protocol for the preventive intervention was developed based on two psychological treatments that have already been proven effective in the treatment of depression, and some evidence was also present on 
reduction of sickness absence. The intensity of the preventive intervention was adapted to the mild level of health complaints of the employees and the focus was mainly on work-related problems. The number of sessions employees received was found to be associated with the outcomes. The results of the study show that those employees who completed the intervention according to the basic part of the protocol (consisting of 7 sessions), as was recommended by the protocol, had the highest decrease in depressive complaints and also the lowest total number of sickness absence days at the end of the follow-up period, compared with employees who did not complete the basic part or who received more than the recommended seven sessions.

This thesis shows that the preventive strategy, consisting of the objective screening instrument, the guidance to the intervention and the preventive intervention, is effective in the prevention of longterm sickness absence and the reduction of depressive complaints in high-risk employees.

\section{Methodological considerations}

\section{Internal validity}

\section{Study design}

In general, the randomized controlled trial is the best study design to examine the effect of a new intervention in relation to the effect of an already existing intervention, care as usual or no intervention. Although problems with attrition are quite common in randomized trials of psychological interventions ${ }^{1,2}$, in the present study data on the primary outcome sickness absence were available for all employees through linkage with the company registers on certified sick leave.

Blinding of participants for the condition they were assigned to was not possible in this study. This is quite common for randomized trials concerning a psychological intervention, but it may have influenced the results. Participants in the control group were aware that they were identified at high risk. As a consequence, these employees may have sought help themselves, which is known as contamination. To study this phenomenon, treatment and medication use during follow-up were inventoried by questions included in the follow-up questionnaires. The questionnaires revealed no significant differences in treatment and medication use between the two groups, indicating that contamination was not likely to have influenced the results.

Another issue concerning psychological interventions is the possible variation in the way individual interventions are performed. Pharmacological and physical interventions can be strictly protocolled resulting in few freedom of how to perform the intervention. A psychological intervention, however, is susceptible to more variation, as it is known that amongst others the relationship between psychologist and participant can influence the results. The relationship between psychologist and participant could for example also have brought about the Hawthorne-effect. The Hawthorne-effect involves the effect that the efficacy of the preventive strategy can be attributed to the attention participants receive, instead of to the specific intervention it self. The Hawthorne-effect may have 
occurred in our study, and this effect may also occur when the preventive strategy would be applied in occupational health care practice, but since the effect would positively influence the results this would not be a problem.

\section{Selection of employees, non-response and selective withdrawal}

Sample selection can have implications for the internal validity of the study, especially if there is selection bias at the moment of screening. The response rate on the screening instrument was $38.2 \%$, which was rather low and also lower than the response rate $(50.2 \%)$ of the previous study conducted in the same company by Kant et al. ${ }^{3}$, which implies that a part of the target population will not be reached. Possible reasons for the low response rate may have been the reorganization of the company during the study or the inclusion of a questionnaire on depressive complaints, which may have put off employees from participating. A non-response analysis of the screening data revealed that respondents and non-respondents did not significantly differ in the total number of sickness absence days at 12 months of follow-up. Respondents had a mean number of 15.4 (SD 41.8) sickness absence days and non-respondents of 16.3 (SD 42.6) days ( $p=0.101$ ). Besides, almost all employees of the banking company were invited to complete the screening instrument. These findings indicate that selective non-response is not likely to have occurred.

Another possible threat for the internal validity of the study concerns the issue of selective withdrawal. Selective withdrawal could have occurred in case of the self-administered questionnaires and in case of participation in the intervention. The response rates of the questionnaires at baseline, 6 and 12 months of follow-up were $71.1 \%, 71.2 \%$ and $64.7 \%$ respectively. For 6 and 12 months of follow-up, respondents and non-respondents could be compared on sickness absence and the scores on BDIII could be compared for the baseline questionnaire to study possible selective withdrawal. With regards to sickness absence, for the intervention group selective withdrawal may have occurred on the 12 month-follow-up questionnaire, since respondents had significantly higher sickness absence duration ( $n=43$; total of 27.40 days) at 12 months of follow-up compared with the non-respondents ( $n=26 ; 13.88$ days). This might indicate that those employees who benefit the most from the intervention did not respond. For the 6 month-follow-up questionnaire no statistically significant difference between respondents and non-respondents was found in their sickness absence at 12 months of follow-up. For the control group a statistically significant difference in total sickness absence duration at 12 months of follow-up between the respondents ( $n=54 ; 42.54$ days) and nonrespondents ( $n=16 ; 8.94$ days) was found for the 6 month-follow-up questionnaire, but not for the 12-month-follow-up questionnaire. The non-respondents might have been those employees who sought help for their complaints themselves, and/or who recovered from the complaints during follow-up. With respect to baseline scores on BDI-II, no significant differences were found between the respondents and non-respondents on both the 6 and 12 month-follow-up questionnaires.

When comparing the intervention completers $(n=46)$ with the non-completers $(n=23)$, no statistically significant difference was found in sickness absence duration at 12 months of follow-up, a mean total number of 24.29 (SD 34.18) sickness absence days was found in the completers versus 31.00 
(57.07) days in the non-completers. The non-completers had about one week longer sickness absence duration compared with the completers, but this still was a much shorter total sickness absence duration than the control group. These findings might suggest that for these employees consultation with the company counselor was already beneficial, but that sickness absence duration might have been even lower if they had participated in the intervention and had completed the intervention. Scores on BDI-II at baseline were comparable between the intervention completers and non-completers, suggesting no selective withdrawal with regards to depressive complaints.

\section{Measurements}

\section{Sickness absence}

Sickness absence was assessed objectively through linkage on an individual level with the company registers on certified sick leave. For every participant in the study, sickness absence data were available. As a result, there was no loss to follow-up in case of sickness absence and the measurement of sickness absence can therefore be considered a valid and reliable method. Sickness absence was assessed from two months after employees completed the screening questionnaire until 20 months of follow-up. Initially, sickness absence was planned to be assessed for 12 months of follow-up. However, since a considerable amount of intervention periods took more than the four months that were determined by the protocol, it was required to extend the follow-up period to realize an actual follow-up period after completion of the interventions of 12 months.

Long-term sickness absence was defined as a sickness absence spell lasting for more than 28 days. In the study on the efficacy of the preventive intervention one of the outcomes was the proportion of employees who had at least one long-term sickness absence spell. Despite the difference of more than $40 \%$ ( $35 \%$ reduction would have been enough according to the power calculation) between the intervention and control group, no statistically significant difference was found. As described earlier, this may have been due to the reduction in sickness absence rates in the Netherlands. However, this may also be explained by the fact that the number of employees who had one or long-term sickness absence spells was counted instead of all long-term sickness absence spells in both groups. Recurrence of long-term sickness absence spells was only found in the control group during followup, not in the intervention group. Then, a borderline significant result was found after 12 months of follow-up ( $p=0.056)$, but still a non-significant result was found at 18 months of follow-up $(p=0.086)$. The continuous variable of total number of sickness absence days seems to better represent actual sickness absence, since many sickness absence spells did not include 28 days, but very close to 28 days, and also many spells were observed with a very long duration of much more than 28 days (up to 429 consecutive sickness absence days), but these spells are counted only once using this method. 


\section{Depressive complaints}

Employees were identified with depressive complaints using the HAD-D. In the follow-up questionnaires, the main measure to assess depressive complaints was the Dutch version of the self-administered Beck Depression Inventory-II (BDI-II). ${ }^{4}$ For the purpose of the study, the golden standard for diagnosis of depression, the SCID-1, was not a feasible option. Therefore, the BDIII was considered a well-established valid and reliable questionnaire to assess the presence and severity of depressive complaints. However, the BDI-II was too extensive and too heavy to include in the screening instrument, since the screening instrument also included the questionnaire to assess the risk of future sickness absence. The HAD-D is a much shorter questionnaire and has been demonstrated a valid questionnaire to identify depressive complaints in the working population. ${ }^{5}$ When the scores of the cases on HAD-D (HAD-D $\geq 8$, indicating mild to severe depressive complaints) were compared with the scores on BDI-II at baseline, $41.3 \%$ of the cases on HAD-D scored in the mild to severe range on BDI-II. The majority of $48.2 \%$ of the cases on HAD-D scored in the no to minimal range of the BDI-II (scores 0 to 10 ). However, the mean BDI-II score was 9.77, thus scores were mainly in the upper part of the lowest category and a reliable change ( $>7$ points) in depressive complaints could still occur. Approximately $10 \%$ of the cases on HAD-D scored in the severe range of the BDI-II, but all scores were in the lower part of the category. Based on these results, it may be concluded that a fairly good match between HAD-D and BDI-II was found and that the HAD-D was an appropriate measure for screening of depressive complaints.

One of the two main aims of the study was to prevent major depression. The concept of depression was defined as a spectrum of depressive complaints, ranging from no depressive complaints to major depression. A score above 19 points indicates mild to severe depressive complaints. A score between 29 and 63 points on BDI-II indicates severe depressive complaints, including major depression at the end of the spectrum. Employees with mild to severe complaints on HAD-D (HAD-D $\geq 8$ ) were selected. The average score on BDI-II at baseline in the intervention group was 17.03 points. The score on $\mathrm{BDI}-\mathrm{Il}$ decreased during follow-up in the intervention group to 12.42 points after 12 months of follow-up. To examine prevention of major depression in more detail, individual scores on BDI$\mathrm{II}$, and in particular high scores on BDI-II, were studied. When looking at individual scores in the intervention group, two employees scored in the severe range of the BDI-II at 12 months of followup, of which the maximum score was 44 points. The baseline score of this person was 42 points. The intervention was probably not intense enough to reduce the depressive complaints, but it is believed that this person did not develop a major depression. The control group included six persons who scored in the severe range of the BDI-II at 12 months of follow-up, of which the highest score was 50 points. Since scores in the intervention group decreased over time and increased in the control group, it is believed that major depression was prevented.

\section{Properties of the screening instrument}

Employees were selected for participation in the RCT by a screening instrument consisting of two screening questionnaires, the Balansmeter to assess the risk of future sickness absence and the 
HAD-D to assess depressive complaints. For the Balansmeter the original cut-off point was used, based on the study by Kant et al, ${ }^{6}$ which dichotomized scores into cases and non-cases. Cases were employees identified at high risk of future sickness absence and non-cases were employees who were identified not at high risk. The cut-off point on HAD-D divided scores into cases, employees with mild to severe depressive complaints, and non-cases, employees with no to mild depressive complaints. Employees who were a case on both questionnaires were selected as eligible candidates for participation in the RCT.

Before the start of the RCT, a pilot study was conducted among a sample of 1,000 employees. The results of the pilot study demonstrated that the overlap of the concepts depressive complaints and risk of future sickness absence was lower than was expected beforehand.

In the development of the Balansmeter, a one-step screening procedure including an instrument with a high specificity was preferred to ensure that the intervention was directed at those employees who actually have an increased risk and that the money would be well-spent on those employees who would benefit the most from the preventive intervention. ${ }^{6}$ Application of more-than-one-step diagnostics would also have been difficult in case of identification of mild complaints and may imply additional non-response and withdrawal. The sensitivity and specificity of HAD-D are both quite high, approximately $80 \% .^{7}$ However, due to the choice for a high specificity of the Balansmeter, this resulted in selection of a too low number of cases for the RCT. Therefore, the effect of a lower cut-off point with a higher sensitivity was studied on the predictive properties. The sensitivity improved, but the specificity decreased to $63.8 \%$ in men and $77.6 \%$ in women, indicating that a considerable number of employees will be classified as being at high risk of future sickness absence, but who will not go on sick leave. Due to the relatively low sensitivity there will also be employees classified as being not at high risk of future sickness absence, but who will actually go on sick leave. When taking into account the consequences of misclassification, no severe adverse effects were expected to occur, since the screening was directed at employees with mild health complaints. Moreover, in case employees ask for help themselves, they receive the available care provided by the occupational health services. Furthermore, an important purpose of the preventive strategy is that it should be applied on a regular basis in the organization. Employees who were classified this time as being not at high risk of future sickness absence, but who actually were at high risk, will probably be identified the next time the preventive strategy is conducted. Of course, the use of different cut-off points on the Balansmeter may be considered depending on for example the target population and the consequences of misclassification, that is, under- or overtreatment.

As mentioned above, for the selection of employees with depressive complaints, a cut-off point on HAD-D of $\geq 8$ points was used. This cut-off point implies that employees with major depressive complaints could have been included. However, in general, employees with major depression are not very likely to be at work and in most cases they are already on sick leave. ${ }^{8}$ Since employees who were on sick leave already at the time of screening were excluded from the study and the relatively low scores on BDI-II at baseline, it was not likely that employees with major depressive complaints were included in the study, but this can not be fully ruled out. 


\section{Time window and follow-up}

An important aspect with regard to the application of the preventive strategy is the time period between the identification of employees at high risk of future sickness absence and with mild depressive complaints and the actual occurrence of long-term sickness absence and development of major depression. Performance of the preventive intervention requires a certain amount of time and the intervention needs a certain follow-up period to become effective. The study proved that a followup period of 18 months was required in men, starting from baseline, to detect a statistically significant difference in long-term sickness absence between employees with depressive complaints who were at high risk versus employees who were not at high risk of future sickness absence. Besides, the results of the RCT showed that depressive complaints in the control group increased during follow-up, but the average score on BDI-II was still in the mild range of the scale. These findings demonstrate that the time period between identification and actual occurrence of long-term sickness absence is sufficient to conduct the preventive intervention. In this study, the efficacy of the preventive intervention on depressive complaints and sickness absence was studied separately. Since the intervention proved to be effective on both outcomes, it is recommended to study the temporal relationship between depressive complaints and sickness absence in more detail. The time window of the study seems to be appropriate to examine this relationship, since sickness absence data are available for a period of 18 months and depressive complaints were assessed at three moments, at baseline and at 6 and 12 months of follow-up. In the development of the preventive intervention it was assumed that the intervention would first reduce depressive complaints and subsequently prevent long-term sickness absence. However, examination of the exact pathway remains subject for future research.

\section{External validity and generalizability}

The effect of the preventive strategy was examined among employees working in a large banking company. The occupational health services performed the organization of the preventive strategy, company counselors performed the guidance to the intervention and psychologists provided the intervention. The type of the company, the type and content of the intervention, the professions included in the study, all these factors contribute to the external validity of the study. A change in one of the factors may result in different outcomes.

Sample selection and the choice of the follow-up period can have implications for the internal validity of the study, but may also have implications for the external validity. In the selection of the study sample, employees who could not speak or read the Dutch language were excluded. Validation of the English version of the Balansmeter and development of an English version of the intervention is required before it can be applied among non-Dutch workers. The time window gives an indication of the minimum amount of time between one period of screening and preventive intervention and the next. The effect of the preventive intervention on sickness absence was still present at 12 months of follow-up for depressive complaints and at 18 months of follow-up for sickness absence duration and might probably have enduring effects. After participation in the intervention, participants are 
expected to have recovered or at least have a reduced risk of sickness absence and reduced depressive complaints, and they are also expected to have better coping mechanisms the next time problems or complaints arise. The final goal of the preventive strategy will be to monitor the employees' risk status over time. However, it is for example unknown how long it takes to develop the high risk of future sickness absence and the mild to severe level of depressive complaints. Therefore, the optimal frequency of conducting the cycle of the preventive strategy in occupational health care practice should be further examined.

\section{Work setting, time period and organizational changes}

The external validity and generalizability of the study also depend on the setting and the time period in which the study was conducted. The study was conducted among employees of a nationwide banking company in the Netherlands, the ABN AMRO Banking Company. The study was conducted in one single company, thereby raising the question whether the employees of this company were representative of office workers in general. The average number of working hours in the company under study was 33.4 (SD 5.31) hours per week, including $73.6 \%$ of fulltime jobs (36 working hours or more). Results of the studies presented in this thesis showed that the population included in the study was a rather heterogeneous population with regard to for example, age, educational level, and the presence of a long-term illness. Characteristic of the company was the rather low sickness absence rates in the years of study, 3.83\% in 2007 and in 3.77\% in 2008. In general, sickness absence rates decreased over the years in the Netherlands, from approximately $5.0 \%$ in 1998 to $4.5 \%$ in 2007 and $4.3 \%$ in $2008 .{ }^{9}$ These low sickness absence rates may have influenced the results. The prevention of sickness absence in our study may have been an underestimation compared with companies that have higher sickness absence rates.

The years the study was conducted included turbulent times for the banking company. The banking company was sold, a merge with two other banking companies took place and many employees were dismissed. These developments may have created a lot of insecurity for the employees, including those employees who participated in the study. Psychological distress and job insecurity for example may have influenced the results of the study. Employees might have been afraid that participation in the study could have consequences for their jobs. However, the employer was nor aware of participation of employees in the study neither of any study results, since privacy was guaranteed by the occupational health services and the researchers. It can be imagined that in a situation of insecurity, employees may become more suspicious and more careful and decide not to participate in the study. This may probably have caused lower response rates on the screening questionnaire. The influence of these organizational changes was not examined in the study. In general, in times of organizational changes sickness absence rates are often lower, because employees are afraid to lose their jobs. ${ }^{10}$ This might indicate that the sickness absence rates of the intervention and control group may have been lower than they would have been in times of a more stabile organization. Nevertheless, no differences between the intervention and control group are expected, since these employees already agreed to participate by completing the screening and baseline questionnaire. 


\section{Quality of care as usual}

The research partner who cooperated in the study was occupational health services Beter, and the banking company under study is one of their clients. The quality of care provided by the occupational health services Beter is known to be of very good quality. All employees of ABN AMRO Bank receive a health check-up every three years, and they also receive the Balansmeter and if necessary a preventive intervention, depending on the cause(s) of the high risk of sickness absence, every three years. Both procedures aim at assessment and prevention of consequences of (work-related) risks on (mental) health. Furthermore, the occupational health services are easy accessible for employees, revealing itself in the high number of consultations per year requested by employees. Many professions are represented at the occupational health services, besides occupational physicians, amongst others company counselors, psychologists and prevention consultants are present. Of the total number of employees (January $2008 \mathrm{~N}=24,313$ ), 2,529 employees contacted the occupational health services in 2008. Of these employees, 851 employees asked help for psychological complaints, such as burnout, depression, and stress-related complaints. The control group of the RCT received care as usual, which included the high quality of care provided by the occupational health services. However, employees in the control group only received care in case of sickness absence or in case they asked for help themselves. Hence, the contrast between the intervention group and the control group may have been reduced. This may have resulted in an underestimation of the effects, when the company under study is compared with other companies who may have a lower quality of occupational health care.

\section{Considerations regarding implementation of the preventive strategy}

Characteristic of the preventive approach described in this thesis, is the fact that many employees identified at high risk did report not to experience health complaints, indicating that these employees were not aware of their status of being at risk at the moment of screening. Besides, many employees who did report to experience health complaints lacked the intention to seek help. These consequences of using a preventive approach were noticeable throughout the RCT, starting from the screening questionnaire, in which the experience of health complaints and help seeking behavior were inventoried. When employees were identified as eligible candidates for participation in the RCT and invited for the consultation with the company counselor, most employees visited the company counselor. However, a considerable number of employees refused to participate in the preventive intervention, because they declared not to recognize their status of being at high risk. This already was a point of interest in the previous study conducted at the same company in $2003 .{ }^{3}$ Therefore, the consultation with the company counselor was introduced in the present study to improve the participation rate. After the consultation with the company counselor, participants who agreed to participate were encouraged to call the psychologist themselves to make an appointment for the first treatment session. Nevertheless, this was observed to be a barrier for many employees, because many employees did not contact the psychologist themselves. Psychologists were therefore instructed to call if the employee did not call within two weeks. Before the actual start of the 
intervention, there were also employees who refused to start participation. Again, reasons such as 'I do not see the usefulness of this treatment' were mentioned. Even when employees started participation in the intervention, a considerable number of employees did not complete the basic part of the intervention as was recommended by the protocol. Besides the experience of health complaints, the nature of the early intervention, which was a psychological intervention, might have deterred employees from participation. Actual participation in early intervention is still an important challenge in research on prevention.

Summarizing the findings with respect to the internal and external validity of the study, we believe that the internal validity of the study can be considered as good. Regarding the external validity, several factors that could have influenced the external validity and generalizability of the study were presented. Several factors were mentioned related to the company the study was conducted at, such as the reorganizations within the company and the quality of the occupational health care. Moreover, aspects with regard to the choice of the study population and the design of the study were described. Many questions can still be asked about the external validity and generalizability of the preventive strategy, and consequently about implementation of the preventive strategy outside the ABN AMRO organization. Are the employees included in the present study, for example, representative of all office workers, or were these office workers more sensitive to develop depressive complaints due to for example the characteristics or the situation of the company? Since this was the first study on prevention of long-term sickness absence and major depression among high-risk employees working in an office working environment, repetition of the study within the same and other companies with office workers is recommended. Besides an office work environment, it may also be considered to conduct the study in other kinds of companies and sectors. The use of the preventive strategy in other kinds of companies, such as health care or industry, requires amongst others a different screening instrument. New modules of the Balansmeter have recently been developed for health care workers and for employees working in industry, but these modules still need to be validated. Replication of the study and further research on the external validity and generalizability will provide further insight in the efficacy of the preventive strategy and may nuance the current insights on the preventive strategy. Based on the current results and the internal validity of the present study, the next paragraphs describe implications for different stakeholders when implementation of the preventive strategy would be considered. Furthermore, recommendations for further improvement of the preventive strategy and for future research are presented. 


\section{Implications for different stakeholders}

\section{Implications for the employee}

The preventive strategy may be an appropriate method to implement in occupational health care practice. Who would exactly benefit from the preventive strategy? First of all the employees may benefit, since participation in the preventive strategy prevents long-term sickness absence, and may consequently prevent social impairment and the risk of work disability. The preventive strategy also reduces depressive complaints. Besides, the intervention may improve general well-being and the ability to better cope with problems in the future.

The lack of experience of health complaints in $31 \%$ of the employees who were identified by the screening instrument as eligible candidates for the RCT raises the question if it is appropriate and acceptable to confront employees with their high risk status. Confronting employees with the risk status as identified by the screening instrument may amongst others cause concern and labeling. Besides, not all employees who are identified at high risk will ultimately go on long-term sickness absence or develop major depression. However, given the relatively mild nature of the complaints, the consequences of under- or overtreatment and the consequences of not using the preventive approach, it was believed that the preventive approach was appropriate and acceptable. The preventive intervention resulted in prevention of long-term sickness absence and reduction of depressive complaints, improvement of general well-being and the ability to learn to deal with the complaints and problems, which can be used henceforth in daily living. Under- and overtreatment depends on the amount of misclassification of the screening instrument. As described earlier, in the paragraph about characteristics of the screening instrument, the choice of a certain cut-off point determines the amount of misclassification and under- and overtreatment of employees identified by the screening instrument. Given the relatively mild health complaints, and the intervention which was specifically developed for mild health complaints, both in case of under- and overtreatment serious adverse effects were not likely to occur.

\section{Implications for the employer}

For employers, the preventive strategy will be most beneficial in the reduction of costs associated with long-term sickness absence. The preventive strategy substantially reduces the total number of sickness absence days and the number of long-term sickness absence spells.

In this thesis, only a rough cost-benefit analysis was performed, nevertheless, to be able to weigh the exact costs of the preventive strategy against the benefits, a more in-depth cost-benefit analysis should be performed to gain more insight in the costs and benefits for all parties involved in the preventive strategy.

The complete process of the preventive strategy is a comprehensive and time-consuming process. Companies have to embrace the idea of exposing their employees to an objective screening process and to motivate their employees to participate. Furthermore, a very important aspect of the use of the preventive strategy is the privacy of the employee. Throughout the study, the privacy of all 
employees needs to be guaranteed by the employer. The employer may not be acquainted with which employees do or do not participate in the preventive intervention. The guidance to the intervention was conducted by company counselors. As a result, the occupational health services were acquainted with which employees were selected for participation in the intervention. This situation is in agreement with the situation in which employees seek help themselves, only the occupational health services are informed but not the employer. For the intervention, privacy was guaranteed as well, since the intervention was conducted by an independent organization. Besides, employees were free to choose if they wanted to receive treatment within or after working hours. Again, only the occupational health services were aware of the participants in the intervention, since they arranged the payments of the intervention in case treatment costs were higher than the costs refunded by the employees' health insurance company.

\section{Implications for occupational health care professionals}

Besides the lack of experience of health complaints by employees, withdrawal from the preventive intervention may also have been caused by the mismatch between the preventive nature of the strategy and the procedure used by health care professionals. In general, health professionals, including company counselors, are trained to work in a demand-driven way. Since many employees reported not to experience health complaints, consultation with the company counselor requires clear explanation and a certain degree of persuasion to encourage employees to participate in the intervention, instead of a demand-driven method. Furthermore, in the phase between the consultation with the company counselor and the start of the intervention, employees were asked to contact the psychologist themselves. In the light of a more proactive approach, it may have been better to let the psychologists contact the employees to make the first appointment. This could have reduced the time between the consultation and start of the intervention and it might have reduced withdrawal. During the intervention, commitment to the intervention might also have been positively influenced by a more proactive procedure of the psychologists, since reasons for ending participation were for example that employees did not see the usefulness of the intervention, or employees did not believe they needed a psychological intervention. After the preventive strategy is completed, the process should be evaluated by the psychologist and the company to make sure the process of the preventive strategy went well and to make sure that the participant benefitted from the intervention. The preventive intervention was conducted by psychologists from a company consisting of a nationwide network of registered psychologists, called Cenzo B.V. This company regularly provides psychological health care for the occupational health services Beter. Cenzo B.V. supported the idea of using the preventive strategy and cooperated throughout the study in the organization and evaluation of the preventive intervention. Psychologists were selected to participate in the study based on their education in CBT and experience with CBT treatment. The participating psychologists received a two-day training program before the start of the intervention and a one-day booster session during the intervention period. To improve participation of employees in the study, it may be considered to advance the booster session and to provide a more extensive training program to practice the use of a proactive approach. 


\section{Implications for occupational health services}

The employer will be responsible for the costs of using the preventive strategy in the organization, but in the present study the occupational health services were responsible for the organization of the preventive strategy within the company. Occupational health services should be prepared for the logistics concerning different activities during the process. The screening step includes mailing, gathering and processing of many screening questionnaires, which is time-consuming and quite expensive. When the screening instruments are processed, the occupational health services should identify the employees at high risk and these employees must be invited for a consultation with the company counselor. When employees agree to participate, the occupational health services should monitor if the employee actually starts participation in the intervention. To improve participation in the preventive intervention in the RCT presented in this thesis, a select group of company counselors was trained to conduct the guidance to the intervention. Sickness absence and work-related problems were viewed to fit best with the job responsibilities of company counselors. In addition to the practical implications, the preventive strategy may above all enable occupational health services to use a more proactive approach to prevent long-term sickness absence and major depression.

\section{Implications for health insurance companies}

In the present study, the health insurance company connected to the banking company agreed to cover the costs for the preventive intervention and if employees had health insurance from a different company, the research project paid the costs. To make the preventive strategy accessible for all employees and since depressive complaints are associated with more frequent use of health services, health insurance companies should consider coverage of the costs of the preventive intervention.

\section{Implications for society}

Besides the expected beneficial effects for amongst others employees and employers, the preventive strategy is expected to be beneficial for society as well. With the use of the preventive strategy, a relatively small number of employees will be selected as eligible candidates for participation in the preventive strategy. However, the selected employees represent the risk bearers for long-term sickness absence. By preventing long-term sickness absence the preventive strategy indirectly prevents work disability and the influx of employees into disability pensions, and with that the preventive strategy is beneficial to labor participation.

\section{Optimalization of the preventive strategy}

Although the preventive intervention proved effective, one may wonder if the preventive strategy as applied in the present study, was organized in the most optimal way. With regards to the screening step, an objective screening instrument was used to select employees. The advantage of an objective instrument is that all employees of the company could be screened and that all employees were approached uniformly. Additionally, employees are allocated to the intervention based on objective 
criteria. Disadvantages are the problem of non-response on questionnaires and withdrawal from the intervention. An advantage of employees who present themselves with health complaints at the occupational health services is that these employees do experience health complaints and seek help for these complaints. However, complaints may have become too serious already. Another option may be that employees are referred to the occupational health services by for example a supervisor, this method is very subjective and depends highly on personal appraisal and judgment, which is eliminated by the use of an objective instrument. An important finding from the present study was that $31 \%$ of the employees who were identified at high risk were not aware of health complaints and their risk status. In this case, the use of an objective screening instrument is the only option to select these employees, but as stated earlier may also result in withdrawal. Moreover, the screening instrument used in the present study consisted of paper questionnaires that were sent to the employees' home addresses. Nowadays, many questionnaires are presented electronically. Response rates might have been higher if electronic questionnaires were used, since employees can more easily decide when and where to complete the questionnaire, at home, at work or somewhere else. Recently, an electronic version of the Balansmeter has been developed. An English version was developed as well, but it needs to be validated before use. This makes it possible to include English speaking employees in the screening. However, an English version of the preventive intervention has not been developed yet.

With respect to the guidance to the intervention, the way the company counselors approached the participants proved to be of great importance for the actual participation of employees in the intervention. For further improvement of the preventive strategy it is believed that company counselors are well-equipped to perform guidance to the intervention, but that the approach should be more proactive in case employees do not see the usefulness of participation in the intervention due to not experiencing health complaints, despite being identified at high risk of future sickness absence and with mild to severe depressive complaints.

Subsequently, the focus is on reflection of the preventive intervention itself. A new protocol, based on the widely used treatments PST and CBT, which have been proven effective in the treatment of depression, was developed for the preventive intervention. Instead of the specific preventive intervention based on PST and CBT, other strategies might be effective as well, such as different types of psychotherapy, counseling, physical training or relaxation techniques. However, so far these kinds of therapy proved to be especially effective in the improvement of perceived health, reduction of symptoms and complaints and improvement of general well-being, but little evidence is available about the effects on future sickness absence and depression. ${ }^{11,12}$

The aim of a protocol is to record all aspects of how and when the intervention should be conducted, and to minimize the amount of variation between individual interventions. The psychologists were trained how to use the protocol. Despite the use of a protocol still some variations could have occurred between individual interventions. The findings of the study demonstrated that the number of sessions received by the participants had an influence on the outcomes depressive complaints and sickness absence. The protocol consisted of a basic part of seven sessions, which was determined 
as being sufficient for the target population of the study, and a specific part of a maximum of five additional sessions, in case the recommended seven sessions were not sufficient. The preventive intervention proved to be effective irrespective of the number of sessions employees received. However, when the number of sessions was examined in more detail, the group of employees who completed the intervention according to the basic part of the protocol showed the best results on the primary outcomes, depressive complaints and sickness absence duration. These findings might suggest that a tailored intervention or a more stepped-care approach may be more effective, but this needs to be further examined. The protocol for the intervention was developed by four experts in the field of psychology. The intervention might have been even more targeted to the study population, if an expert of the company was involved, someone with specific knowledge on what work factors specifically related to the type of jobs within the company can be expected to be associated with depressive complaints and long-term sickness absence and which should be handled in the intervention. The intervention was provided by psychologists from an independent organization called Cenzo B.V. Since the intervention consisted of PST and CBT, psychologists are the proper profession to deliver the intervention, however, as mentioned before, the need for a more proactive treatment style should be emphasized.

\section{Future research}

Although this thesis gives answers to the research questions as described in the introduction, the study generated many new research questions as well. Therefore, some recommendations for future research are formulated. The previous paragraph described suggestions for further improvement of the preventive strategy itself. This paragraph presents suggestions for future research when the preventive strategy is viewed in a broader perspective, such as application in different settings and populations.

With regard to the screening step of the preventive strategy, the screening instrument Balansmeter was originally developed for employees working in an office work environment. Recently, separate Balansmeter modules have been developed for two different sectors, for the industry and for health care. The predictive properties and the sensitivity and specificity of these new modules should be evaluated and externally validated in different companies.

The preventive strategy examined in the present study focused on employees both at high risk of future sickness absence and with mild to severe depressive complaints. However, the overlap between the concepts of risk of future sickness absence and mild to severe depressive complaints was found to be lower than expected. Many employees were identified either at high risk of future sickness absence or with mild to severe depressive complaints. The results of the study described in chapter 2 demonstrated that even mild to severe levels of depressive complaints were found to be associated with future long-term sickness absence. Therefore, for future research, it may be considered to aim screening at each of the three sub groups and offer them all the preventive intervention, since the intervention was found to reduce depressive complaints and to prevent longterm sickness absence as well. When the preventive intervention was developed the assumption 
was that the intervention would first reduce depressive complaints and subsequently prevent longterm sickness absence. However, the exact pathway of how the intervention acted upon depressive complaints and sickness absence and the interaction between both concepts, should be investigated in more detail. For further improvement of the intervention, it is also important to know if the intervention reduces both (the risk of) long-term sickness absence and depressive complaints, or that a reduction of depressive complaints consequently results in a reduction of (the risk of) sickness absence or vice versa. Currently, research is conducted on the ethical considerations concerning the use and implementation of the preventive strategy in occupational health care. Effects of the preventive strategy on for example labeling should be studied, since employees are identified by a screening instrument as being at high risk, but they may not experience this status themselves.

The preventive intervention proved effective as described earlier. However, an in-depth evaluation of the costs versus benefits of the preventive strategy should be conducted. Future research may also focus on for example the impact on health care use among those employees who are identified at high risk.

Depressive complaints are known to be strongly associated with other mental health complaints, such as anxiety and stress-related symptoms, ${ }^{13}$ which are prevalent conditions among employees as well. CBT and PST have been found to be effective treatments for anxiety and stress-related symptoms as well. Therefore, using the preventive strategy for a combination of depressive complaints and anxiety or stress-related symptoms might be considered.

\section{Conclusion}

This thesis shows that employees with a high risk of future sickness absence and with mild to severe depressive complaints can be selected by means of an objective screening instrument. In addition, a new preventive intervention specifically developed for the target population proved effective in the prevention of long-term sickness absence and reduction of depressive complaints. Although many questions need to be answered before considering large-scale implementation, the results of this thesis clearly showed that the preventive strategy appears to be a promising tool for use in occupational health care. 


\section{References}

1. Hewitt CE, Kumaravel B, Dumville JC, Torgerson DJ. Assessing the impact of attrition in randomized controlled trials. J Clin Epidemiol 2010;63:1264-70.

2. Kwan BM, Dimidjian S, Rizvi SL. Treatment preference, engagement, and clinical improvement in pharmacotherapy versus psychotherapy for depression. Behav Res Ther 2010;48:799-804.

3. Kant I, Jansen NW, van Amelsvoort LG, van Leusden R, Berkouwer A. Structured early consultation with the occupational physician reduces sickness absence among office workers at high risk for long-term sickness absence: a randomized controlled trial. J Occup Rehab 2008;18:79-86.

4. Beck AT, Steer RA, Brown GK. Beck Depression Inventory second edition - Manual. San Antonio TX: The psychological Corporation, 1996.

5. Andrea H, Bultmann U, Beurskens AJ, Swaen GM, van Schayck CP, Kant IJ. Anxiety and depression in the working population using the HAD Scale--psychometrics, prevalence and relationships with psychosocial work characteristics. Soc Psychiatry Psychiatr Epidemiol 2004;39:637-46.

6. Kant IJ, Jansen NW, van Amelsvoort LG, Swaen GM, van Leusden R, Berkouwer A. Screening questionnaire Balansmeter proved successful in predicting future long-term sickness absence in office workers. J Clin Epidemiol 2009;62:408-14.

7. Bjelland I, Dahl AA, Haug TT, Neckelmann D. The validity of the Hospital Anxiety and Depression Scale. An updated literature review. J Psychosom Res 2002;52:69-77.

8. Rytsala HJ, Melartin TK, Leskela US, Sokero TP, Lestela-Mielonen PS, Isometsa ET. Predictors of long-term work disability in Major Depressive Disorder: a prospective study. Acta Psychiatr Scand 2007;115:206-13.

9. Statistics Netherlands. National statistics on sick leave, frequency, period of absence. Heerlen/Voorburg, the Netherlands. Available at http://www.statline.cbs.nl. Accessed at 11 June 2008.

10. Stegeman H. De conjunctuurgevoeligheid van ziekteverzuim [Sickness absence and sensitivity to market conditions]. Den Haag: Cultureel Planbureau Den Haag, 2005.

11. Duijts SF, Kant IJ, van den Brandt PA, Swaen GM. Effectiveness of a preventive coaching intervention for employees at risk for sickness absence due to psychosocial health complaints: results of a randomized controlled trial. J Occup Environ Med 2008;50:765-76.

12. van der Klink JJ, Blonk RW, Schene AH, van Dijk FJ. The benefits of interventions for work-related stress. Am J Public Health 2001;91:270-6.

13. Kessler RC. The epidemiology of psychiatric comorbidity. In Tsaung MT, Tohen M and Zahner GEP (Eds.). Textbook in psychiatric epidemiology ( $\mathrm{p}$ 179-97).New York: Wiley; 1995. 

Summary 

Long-term sickness absence constitutes a major public health and economical problem in Western societies, as described in chapter 1 of the thesis. Long-term sickness absence has many consequences, for employees, their families, work-places and for society. Despite the reduction of sickness absence prevalence between 1994 and 2010, sickness absence still remains a major problem in terms of costs, labor participation and social consequences. Long-term sickness absence constitutes a source of marked distress and social impairment for the employee and is also associated with high costs for employers and society. In particular long-term sickness absence contributes considerably to the total days lost and it is the rather small population of employees with long-term sickness absences who account for the majority of costs.

Musculo-skeletal complaints and mental health complaints together account for over $70 \%$ of all certified sick-leave in the Netherlands. In most high-income counties mental health complaints now are the leading cause of sickness absence, since mental health complaints are associated with prolonged sickness absence spells. Depressive complaints constitute a substantial part of the mental health complaints in the working population. Especially major depression, but also depressive complaints are known to be associated with (future) long-term sickness absence. The effects of treatment and rehabilitation of employees on sick leave due to mental health complaints including depressive complaints are still limited, since a reduction of symptoms does not necessarily lead to work resumption. Therefore, it was hypothesized that a preventive strategy aimed at prevention of long-term sickness absence and major depression may be more effective.

The general aim of the thesis was to examine if a preventive strategy aimed at employees who are at high risk of future sickness absence and who have a mild level of depressive complaints is effective in the prevention of long-term sickness absence and major depression.

Based on the general aim, two research questions are formulated in this thesis. The first research question is: 'Is it possible to identify employees at high risk of future sickness absence and with mild to severe depressive complaints?' The second research question is: 'Is a preventive intervention, specifically developed for employees who are at high risk of future sickness absence and who have mild to severe depressive complaints, effective in the prevention of long-term sickness absence and major depression?' Both research questions are studied in a randomized controlled trial (RCT), conducted among employees working at a large banking company in the Netherlands, the ABN AMRO banking company.

The basic assumption underlying the questions of this thesis was that depressive complaints are associated with future long-term sickness absence. From the literature it is known that depression is associated with sickness absence, however, information on the relationship between milder levels of depressive complaints and (long-term) sickness absence is scarce. To study this relationship data from the Maastricht Cohort Study on Fatigue at Work were used. Gender stratified logistic regression analyses were used to study the cross-sectional relationship between depressive complaints and sickness absence at the time the employees completed the baseline questionnaire. Gender stratified 
Poisson regression analyses were conducted to study the longitudinal relationship between depressive complaints and sickness absence duration over ten months of follow-up. The results, as presented in chapter 2 , showed a strong cross-sectional association between mild and moderate-tosevere levels of depressive complaints and sickness absence. In the longitudinal analyses, mild and moderate-to-severe levels of depressive complaints were found to be associated with longer sickness absence duration when compared with employees scoring in the normal range of the scale. In both men and women, the risk of longer duration of sickness absence increased as the level of depressive complaints increased. In men, the mean number of sickness absence days was approximately twice as high for employees with mild depressive complaints compared with employees scoring in the normal range of the scale, and approximately 2.5 times higher for moderate-to-severe depressive complaints. Women with mild depressive complaints had approximately 2.5 times more sickness absence days and moderate-to-severe depressive complaints approximately two times more absence days compared with women scoring in the normal range. However, this latter result was not statistically significant. In both men and women, moderate-to-severe levels of depressive complaints were associated with a shorter time to onset of the first sickness absence spell. This study confirmed that even mild levels of depressive complaints are associated with future long-term sickness absence, which further underscores the importance of a preventive strategy.

A prerequisite for the preventive strategy is the ability to identify employees at high risk of future sickness absence and with mild to severe depressive complaints. In chapter 3 , the predictive properties of the screening instrument, consisting of a questionnaire to identify employees at high risk of future sickness absence, called Balansmeter (BM), and a questionnaire to identify employees with mild to severe depressive complaints, the depression scale of the Hospital Anxiety and Depression Scale, were studied. First, the predictive properties of the BM were studied in the present study population, since sickness absence prevalence decreased between the years the BM was externally validated and the present study. The predictive properties for the risk of long-term sickness absence, which was defined as sickness absence duration of more than 28 consecutive calendar days, remained intact. Second, the predictive value of having mild to severe depressive complaints compared with having no to mild depressive complaints was examined, since depressive complaints are associated with sickness absence, as described previously in chapter 2 . Male (female) employees with mild to severe depressive complaints had a 3.13 (2.45) times higher risk of long-term sickness absence compared with male (female) employees with no to mild depressive complaints. When the BM was applied among employees with depressive complaints, the predictive properties of the screening instrument increased to a relative risk of 5.23 in men and 3.87 in women. However, a rather low number of cases was selected by the BM. When the effect of a cut-off point on the BM with a higher sensitivity was studied, the predictive properties remained more or less the same, but the number of cases increased. Male (female) employees with mild to severe depressive complaints who were identified by the BM at high risk had a 4.88 (3.80) times higher risk of long-term sickness absence compared with employees without mild to severe depressive complaints who were not identified at high risk 
by the BM. Nevertheless, the use of the lower cut-off point resulted in a decrease in specificity of the $\mathrm{BM}$ to $64.9 \%$ in men and $78.2 \%$ in women. The findings of this study demonstrate that the screening instrument proved to be effective in identification of the target population of the RCT.

The preventive strategy focused on employees with relatively mild health complaints and a high risk for a future event. The selection of relatively healthy employees may involve certain difficulties. From literature it is known that a consequence of the choice of a preventive approach might be that employees identified at high risk by the screening instrument do not all report to experience health complaints at the moment of completing the screening questionnaire. This might result in employees who are not motivated to participate in the preventive intervention. The experience of health complaints is also known to be the most important factor for help seeking behavior and it was therefore expected to have an influence on actual participation in the preventive intervention. Therefore, the experience of health complaints and help seeking behavior became subject of the present study. Although certain measures were taken before the start of the study to optimize participation in the intervention, such as consultation with a company counselor before the start of the intervention, still many employees did not participate in the intervention. The experience of health complaints and the intention to seek help among the target population of the RCT was studied in chapter 4 . Of the 211 employees who were identified at high risk of future sickness absence and with mild to severe depressive complaints by the screening instrument, 140 (69\%) employees reported to experience health complaints. As a result, still $31 \%$ of the employees did not report to experience health complaints. Subsequently, 105 of the 140 employees who experienced health complaints reported that they already sought help for these complaints. This mainly included a visit to the general practitioner. Of the employees who had not sought help, only $50 \%$ reported to have the intention to seek help for the health complaints. This study quantified the experience of health complaints and help seeking behavior of the target population of the RCT. The results give more insight in the considerations of employees who are selected by a screening instrument and the decision to participate or not participate in the preventive intervention.

Chapter 5 describes the efficacy of a preventive intervention, which was specifically developed for employees who were identified at high risk of future sickness absence and with mild to severe depressive complaints, on the prevention of long-term sickness absence and major depression. Employees were identified at high risk of sickness absence and with mild to severe depressive complaints by the screening instrument $(n=211)$, of which the predictive properties were examined and described in chapter 3 . Eligible candidates for the RCT were randomized into the preventive intervention group $(n=69)$ or into the control group $(n=70)$, receiving care as usual. Employees received self-administered questionnaires at six and twelve months of follow-up. Objective sickness absence data were gathered for 12 months of follow-up. Since a substantial part of the interventions took longer than four months, as was prescribed by the protocol, the follow-up period of sickness absence was extended to 18 months of follow-up, to realize an actual follow-up period of one year. 
The primary outcomes of the RCT are long-term sickness absence and depressive complaints. The intention to treat analyses showed a substantial and statistically significant difference between the intervention (score of 12.8 points on BDI-II at 6 months and 12.4 points at 12 months) and control group (score of 16.3 points on BDI-II at 6 months and 16.7 at 12 months) in depressive complaints at 6 and 12 months of follow-up (for 6 months $p=0.001$ and for 12 months $p=0.005$ ). Intention to treat analyses also showed a substantial and statistically significant difference between the intervention (27.5 sickness absence days) and control group (50.8 sickness absence days) in total sickness absence duration after 12 months of follow-up ( $p=0.017)$. In the per-protocol analyses, employees who received at least one treatment session were included in the intervention group. Even more pronounced results were found compared with the intention to treat analyses. In these analyses a statistically significant difference between the intervention (33.1 sickness absence days) and control group (62.6 sickness absence days) was found for sickness absence duration at 18 months of followup as well $(\mathrm{p}=0.021)$. The proportion of employees with long-term sickness absence spells ( $>28$ days) showed a $40 \%$ lower number of long-term sickness absence spells in the intervention group compared with the control group, which was more than calculated in the power calculation (35\%), however, results failed to reach statistical significance, probably due to the low sickness absence rates in the company. This study shows that the preventive intervention, specifically developed for the target population was effective in the prevention of long-term sickness absence and the reduction of depressive complaints.

The preventive intervention was conducted by psychologists according to the newly developed protocol. Despite the use of the protocol, some variation between individual interventions could have occurred. Three main factors were identified that could have varied between individual interventions. The influence of these three factors, that is, the duration of the intervention period, protocol execution by the psychologists and the number of sessions employees received, on the efficacy of the preventive intervention was described in chapter 6 . The duration of the intervention period and protocol execution by the psychologists were not associated with the outcomes, whereas the number of sessions employees received was associated with the outcomes. The protocol consisted of a basic part of seven sessions, and if seven sessions were not sufficient, the protocol provided a maximum of five additional sessions, the specific part of the protocol. Seven sessions were recommended by the protocol, since the basic part of the intervention was expected to fit with the relatively mild level of health complaints. The actual number of sessions employees received was determined by the employee in consultation with the psychologist. When the study was finished, the results revealed that a considerable number of employees did not complete the basic part of the intervention $(n=17)$. Moreover, there was also a considerable number of employees who received more than the recommended seven sessions $(n=19)$. Ten employees completed the intervention according to the basic part of the protocol. The preventive intervention proved to be most effective in those employees who completed the intervention according to the basic part of the protocol. Employees who completed the intervention according to the basic part of the protocol showed the 
most improvement in depressive complaints at 6 and at 12 months of follow-up, compared with employees who did not complete the basic part of the intervention or who received more than seven sessions. Employees who completed the intervention according to the basic part of the protocol also had the lowest mean total number of sickness absence days when a follow-up period of 12 months was considered. Nevertheless, these findings do not imply that receiving seven sessions in it self leads to better outcomes. Besides, the power of this study was rather low. Future research should further examine the optimal number of sessions for each individual participant.

In the general discussion, chapter 7, the main findings of the study are described and discussed in terms of methodological considerations, considerations regarding implementation of the preventive strategy, implications for different stakeholders, suggestions for optimalization of the preventive strategy and recommendations for future research. This thesis shows that the preventive strategy is an effective method. The screening instrument is able to identify employees at high risk of future sickness absence and with mild to severe depressive complaints. The preventive intervention proved to be effective in the prevention of long-term sickness absence and reduction of depressive complaints. For the implementation of the preventive strategy in occupational health care practice, several points of interest should be kept in mind, such as that the preventive strategy is a comprehensive and timeconsuming process, in which many parties are involved. Special attention should be given to the step of guidance of selected employees to participation in the preventive intervention, since experience of health complaints and motivation to participate in the intervention may lack due to the focus on relatively mild health complaints. From the perspectives of the employee, employer, health insurance companies and society, the preventive strategy is expected to be beneficial. However, future research should amongst others examine the cost-effectiveness of the preventive strategy more thoroughly as well as the use of the preventive strategy in different settings and (target) populations. 

Samenvatting 

Langdurig ziekteverzuim vormt een groot probleem voor de volksgezondheid en is tevens een groot economisch probleem in veel Westerse landen, zoals beschreven staat in hoofdstuk 1 van dit proefschrift. Langdurig ziekteverzuim heeft vele negatieve gevolgen voor onder andere werknemers, hun families, bedrijven en ook voor de samenleving. Ondanks de afname van het ziekteverzuim tussen 1994 en heden, blijft langdurig ziekteverzuim een belangrijk probleem in termen van kosten, arbeidsparticipatie en sociale gevolgen. Voor de werknemer betekent langdurig ziekteverzuim een bron van stress en sociale beperkingen, voor de werkgever en de samenleving brengt verzuim voornamelijk hoge kosten met zich mee. Vooral langdurig ziekteverzuim draagt aanzienlijk bij aan het totaal aantal werkdagen dat verloren gaat en is het met name deze relatief kleine populatie van werknemers met langdurig ziekteverzuim die verantwoordelijk is voor de meerderheid van de kosten.

Aandoeningen van het bewegingsapparaat en mentale gezondheidsklachten zijn samen verantwoordelijk voor meer dan $70 \%$ van het geregistreerde ziekteverzuim in Nederland. Op dit moment vormen mentale gezondheidsklachten de belangrijkste oorzaak van ziekteverzuim in de meeste welvarende landen, vanwege de vaak zeer lange verzuimduur die gepaard gaat met mentale klachten. Depressieve klachten vormen een belangrijk aandeel van deze mentale gezondheidsklachten in de beroepsbevolking. Van depressie, maar ook van depressieve klachten is bekend dat zij geassocieerd zijn met (toekomstig) langdurig ziekteverzuim. De effecten van behandeling en reïntegratie van werknemers die met ziekteverzuim zijn vanwege mentale gezondheidsklachten, inclusief depressieve klachten, zijn echter nog altijd beperkt. Dit komt doordat een afname van de symptomen niet automatisch werkhervatting hoeft te betekenen. Een preventieve benadering die zich richt op preventie van langdurig ziekteverzuim en depressie wordt daarom verondersteld effectiever te zijn dan behandeling en reïntegratie van werknemers die reeds aan het verzuimen zijn.

Het doel van het proefschrift is om te bestuderen of een preventieve benadering, die zich richt op werknemers die een hoog risico hebben op toekomstig verzuim en die tevens milde tot ernstige depressieve klachten hebben, effectief is om langdurig ziekteverzuim en depressie te kunnen voorkomen.

Op basis van dit doel zijn twee onderzoeksvragen geformuleerd. De eerste onderzoeksvraag is: 'Is het mogelijk om werknemers met een hoog risico op toekomstig ziekteverzuim en met milde tot ernstige depressieve klachten, maar die wel nog aan het werk zijn, op te sporen?' De tweede onderzoeksvraag is: 'Is een preventieve interventie, die speciaal ontwikkeld is voor werknemers met een hoog risico op toekomstig verzuim en met milde tot ernstige depressieve klachten, effectief in het voorkomen van langdurig ziekteverzuim en depressie?'. Beide vragen zijn onderzocht in een zogeheten gerandomiseerd gecontroleerd onderzoek, dat uitgevoerd werd bij kantoormedewerkers van een grote bank in Nederland, namelijk ABN AMRO Bank.

De basisaanname van het onderzoek dat beschreven wordt in dit proefschrift is dat depressieve klachten verband houden met toekomstig langdurig ziekteverzuim. Uit de literatuur is bekend dat 
depressie geassocieerd is met ziekteverzuim, maar informatie over de relatie tussen mildere niveaus van depressieve klachten en (langdurig) ziekteverzuim is spaarzaam. Om deze relatie te onderzoeken is gebruik gemaakt van data van de Maastrichtse Cohort Studie over vermoeidheid in de arbeidssituatie. Logistische regressieanalyses werden uitgevoerd om de cross-sectionele relatie te bestuderen tussen depressieve klachten en ziekteverzuim ten tijde van het invullen van de screeningsvragenlijst. Poisson regressieanalyses werden uitgevoerd om de longitudinale relatie tussen depressieve klachten en ziekteverzuimduur te onderzoeken over een periode van tien maanden. De analyses werden apart uitgevoerd voor mannen en vrouwen. De resultaten van deze studie, zoals beschreven in hoofdstuk 2 , laten een sterk cross-sectioneel verband zien tussen zowel milde als matig tot ernstige depressieve klachten en ziekteverzuim. Uit de resultaten van de longitudinale analyses bleek dat matig tot ernstige depressieve klachten geassocieerd zijn met een langere ziekteverzuimduur in vergelijking met werknemers die geen tot milde klachten rapporteerden in de depressievragenlijst. Voor zowel mannen als vrouwen neemt het risico op een langere ziekteverzuimduur toe bij een toename van de klachten. Bij mannen was het gemiddelde aantal dagen verzuim ongeveer twee maal zo hoog voor werknemers met milde depressieve klachten dan voor werknemers die geen tot milde klachten scoorden op de depressie vragenlijst, en het risico was zelfs 2.5 keer zo groot voor werknemers met matig tot ernstige depressieve klachten. Vrouwen met milde depressieve klachten hadden 2.5 keer meer verzuimdagen en de werknemers met matig tot ernstige depressieve klachten hadden twee keer zoveel verzuimdagen in vergelijking met werknemers die geen tot milde klachten rapporteerden. Echter, dit laatste resultaat bleek niet statistisch significant te zijn. Voor zowel mannen en vrouwen waren matig tot ernstige depressieve klachten geassocieerd met een kortere tijd tot het begin van de eerste ziekteverzuimperiode. Deze studie heeft bevestigd dat ook milde niveaus van depressieve klachten een verband hebben met toekomstig langdurig ziekteverzuim, hetgeen het belang van de keuze voor een preventieve benadering onderstreept.

Een vereiste om de preventieve benadering toe te kunnen passen is de mogelijkheid om werknemers met een hoog risico op toekomstig verzuim en met milde tot ernstige depressieve klachten te identificeren. In hoofdstuk 3 zijn de voorspellende kwaliteiten van een screeningsinstrument om toekomstig langdurig ziekteverzuim te voorspellen onderzocht. Het screeningsinstrument bestaat uit een vragenlijst om werknemers met een hoog verzuimrisico op te sporen, de Balansmeter genaamd (BM), en tevens uit een vragenlijst om werknemers met milde tot ernstige depressieve klachten op te sporen. Ten eerste werden de voorspellende kwaliteiten van de BM bestudeerd waarbij deze werd toegepast binnen de totale populatie van het onderzoek. De prevalentie van ziekteverzuim is gedaald in de tijd tussen de externe validatie van de BM, die ook binnen ABN AMRO Bank plaatsvond, en het huidige onderzoek, en daarom werd eerst gekeken of de voorspellende kwaliteiten gelijk zijn gebleven. De voorspellende kwaliteiten van de BM voor het risico om langdurig te gaan verzuimen, waarbij langdurig verzuim gedefinieerd was als een periode van meer dan 28 dagen aaneengesloten verzuim, bleken nog altijd goed te zijn. Ten tweede werd de voorspellende waarde van het hebben van milde tot ernstige depressieve klachten bekeken in vergelijking met 
het hebben van geen tot milde depressieve klachten, omdat depressieve klachten ook geassocieerd zijn met ziekteverzuim, zoals eerder beschreven in hoofdstuk 2. Mannen (vrouwen) met milde tot ernstige depressieve klachten hebben een 3.13 (2.45) keer hoger risico op langdurig ziekteverzuim dan mannen (vrouwen) met geen tot milde depressieve klachten. Toepassing van de BM binnen een groep van werknemers met mild tot ernstige depressieve klachten leidde tot een toename van het relatieve risico tot 5.23 voor mannen en 3.87 voor vrouwen. Echter, dit resulteerde in een laag aantal werknemers dat geselecteerd werd door de BM met een hoog risico op verzuim en tevens met mild tot ernstige depressieve klachten. Daarom werd vervolgens het effect van een lager afkappunt op de BM, een afkappunt met een hogere sensitiviteit, onderzocht op de voorspellende kwaliteiten. De voorspelling van langdurig verzuim bleef min of meer gelijk, maar het aantal cases dat geselecteerd werd nam toe. Mannen (vrouwen) met milde tot ernstige depressieve klachten, die geïdentificeerd waren door de BM met een hoog risico op verzuim, hadden een 4.88 (3.80) keer groter risico op langdurig ziekteverzuim dan werknemers die geen milde tot ernstige depressive klachten en die geen hoog risico op verzuim hebben. Het toepassen van dit lagere afkappunt resulteerde wel in een afname van de specificiteit van de BM tot $64.9 \%$ voor mannen en $78.2 \%$ voor vrouwen. De bevindingen van deze studie laten zien dat het screeningsinstrument effectief is gebleken in het opsporen van de doelpopulatie van het gerandomiseerd gecontroleerd onderzoek (RCT).

De preventieve benadering is gericht op werknemers met relatief milde gezondheidsklachten en een hoog risico op het ontwikkelen van een gebeurtenis in de toekomst, namelijk het optreden van ziekteverzuim. De selectie van relatief gezonde werknemers kan bepaalde moeilijkheden met zich meebrengen. Uit eerder onderzoek is gebleken dat een gevolg van de keuze voor de preventieve benadering kan zijn dat werknemers die geselecteerd werden door het screeningsinstrument rapporteren dat zij geen gezondheidsklachten ervaren op het moment van het invullen van de vragenlijst. Het niet ervaren van gezondheidsklachten kan er onder andere toe leiden dat mensen niet gemotiveerd zijn om deel te nemen aan de preventieve interventie. Uit de literatuur is tevens bekend dat het ervaren van gezondheidsklachten de meest belangrijke factor voor hulpzoekend gedrag vormt. Dit gegeven was de aanleiding om te verwachten dat het al dan niet ervaren van gezondheidsklachten van invloed zou zijn op de deelname aan de interventie. Deze gegevens waren aanleiding om in het onderzoek extra aandacht aan te besteden aan het ervaren van gezondheidsklachten en hulpzoekend gedrag. Ondanks het feit dat er voor de start van de studie maatregelen waren genomen om deelname aan de interventie te optimaliseren, zoals een gesprek met een bedrijfsmaatschappelijk werker als voorbereiding op de interventie, waren er toch relatief veel werknemers die niet deelnamen aan de interventie. Het ervaren van gezondheidsklachten en het hulpzoekend gedrag van de doelpopulatie van het onderzoek werd bestudeerd in hoofdstuk 4. Van de 211 werknemers die geïdentificeerd waren met een hoog risico op toekomstig ziekteverzuim en met milde tot ernstige depressieve klachten, gaven 140 (69\%) werknemers aan gezondheidsklachten te ervaren, met als gevolg dat $31 \%$ van de werknemers rapporteerde geen gezondheidsklachten te ervaren. Vervolgens gaven 105 van de 140 werknemers die gezondheidsklachten rapporteerden aan 
dat zij al hulp hadden gezocht voor de gezondheidsklachten. Het merendeel van deze werknemers bezocht de huisarts. Van de werknemers die nog geen hulp gezocht hadden voor hun klachten gaf slechts $50 \%$ aan dat ze de intentie hadden om hulp te gaan zoeken. Met deze studie is getracht het ervaren van gezondheidsklachten en het hulpzoekend gedrag van de doelpopulatie van het onderzoek te kwantificeren. De resultaten geven meer inzicht in de achtergrond van de keuze van werknemers die geselecteerd zijn door middel van een objectief screeningsinstrument om al dan niet deel te nemen aan de aangeboden preventieve interventie.

Hoofdstuk 5 beschrijft de effectiviteit van de preventieve interventie, die specifiek ontwikkeld is voor werknemers met een hoog risico op toekomstig ziekteverzuim en die milde tot ernstige depressieve klachten hebben, in het voorkomen van langdurig ziekteverzuim en depressie. Werknemers $(n=211)$ werden geselecteerd door middel van een objectief screeningsinstrument, waarvan de voorspellende kwaliteiten werden onderzocht en waarvan de resultaten beschreven staan in hoofdstuk 3 . Geschikte kandidaten voor deelname aan het gerandomiseerd gecontroleerd onderzoek werden 'at random' toegewezen aan de preventieve interventiegroep $(n=69)$ of aan de controlegroep $(n=70)$, die de standaard arbozorg ontving. Werknemers ontvingen vragenlijsten na zes en twaalf maanden follow-up. Objectieve verzuimgegevens werden in eerste instantie verzameld voor een periode van twaalf maanden. Echter, een aanzienlijk aantal interventies duurde langer dan de door het protocol voorgeschreven vier maanden, daarom was het nodig om de perioden van het verzamelen van verzuimgegevens te verlengen tot 18 maanden. Op deze manier waren voor de deelnemers aan de interventie verzuimgegevens beschikbaar voor een periode van circa twaalf maanden nadat de interventie was afgerond. De primaire uitkomstmaten van het onderzoek zijn langdurig ziekteverzuim en depressieve klachten. De intention-to-treat analyses laten een aanzienlijk en statistisch significant verschil zien tussen de interventiegroep (12.8 punten op de BDI-II na 6 maanden en 12.4 punten na 12 maanden) en de controlegroep (16.3 punten op de BDI-II na 6 maanden en 16.7 punten na 12 maanden) in de ernst van de depressieve klachten, na zowel zes als twaalf maanden follow-up (voor 6 maanden $p=0.001$ en voor 12 maanden $p=0.005)$. De intention-to-treat analyses laten ook een aanzienlijk en statistisch significant verschil zien tussen de interventiegroep ( 27.5 verzuimdagen) en de controlegroep (50.8 verzuimdagen) in de totale ziekteverzuimduur bekeken over twaalf maanden follow-up ( $p=0.017$ ). Voor de per-protocol analyses werden werknemers van de interventiegroep meegenomen in de analyse wanneer zij minimaal één behandelsessie hadden ontvangen. De resultaten van deze analyse laten nog sterkere effecten zien dan de resultaten van de intentionto-treat analyse. Naast een statistisch significant verschil na 12 maanden follow-up, werd er tevens een statistisch significant verschil gevonden tussen de interventie- (33.1 verzuimdagen) en de controlegroep (62.6 verzuimdagen) in totale ziekteverzuimduur na een periode van 18 maanden follow-up ( $p=0.021$ ). De proportie van werknemers met langdurig ziekteverzuim ( $>28$ dagen) bleek $40 \%$ lager te zijn in de interventiegroep in vergelijking met de controlegroep, wat meer was dan het percentage wat berekend was voor de power-calculatie (35\%). Toch werd er geen statistisch significant verschil gevonden, waarvoor de lage prevalentie van ziekteverzuim in het bedrijf een 
verklaring zou kunnen zijn. Deze studie toont aan dat de preventieve interventie effectief is in het voorkomen van langdurig ziekteverzuim en het reduceren van de ernst van depressieve klachten.

De preventieve interventie werd uitgevoerd door psychologen aan de hand van een nieuw ontwikkeld behandelprotocol. Ondanks de toepassing van een protocol kan er toch enige variatie in de individuele interventies zijn opgetreden. Drie belangrijke factoren, die tot variaties tussen de interventies geleid kunnen hebben werden geïdentificeerd, te weten de totale duur van de interventie, de mate van handhaving van het protocol door de psychologen en het aantal sessies dat werknemers hebben ontvangen. De invloed van deze drie factoren op de effectiviteit van de preventieve interventie staat beschreven in hoofdstuk 6. De totale duur van de interventie en handhaving van het protocol door de psychologen blijken geen invloed te hebben op de uitkomsten, terwijl het aantal sessies dat werknemers hebben ontvangen wel een duidelijk verband laat zien met de uitkomsten. Het protocol bestaat uit een basisdeel van zeven sessies, en wanneer zeven sessies niet voldoende waren voorzag het protocol in maximaal vijf extra sessies, het specifieke deel van het protocol. Het volgen van het basisdeel van de interventie werd aanbevolen door het protocol, omdat het basisdeel speciaal ontwikkeld werd voor de relatieve milde gezondheidsklachten van de deelnemers. Het daadwerkelijke aantal sessies dat de werknemers ontvingen werd echter bepaald door de werknemer zelf, in overleg met de psycholoog. Nadat de studie afgerond was, bleek dat een aanzienlijk aantal deelnemers het basisdeel van de interventie niet hebben afgemaakt, dus dat zij minder dan zeven sessies hadden gevolgd $(n=17)$. Daarnaast was er ook een aanzienlijk aantal deelnemers dat meer dan de aanbevolen zeven sessies heeft ontvangen, dus ook het specifieke deel van de interventie of een deel daarvan $(n=19)$. Tien deelnemers volgden het basisdeel van de interventie, zoals voorgeschreven. De resultaten van deze studie laten zien dat de preventieve interventie het meest effectief blijkt te zijn bij werknemers die de zeven sessies van het basisdeel van de interventie ontvingen. Deze werknemers hadden de laagste score op depressieve klachten na zes en twaalf maanden follow-up, in vergelijking met werknemers die het basisdeel niet hadden afgemaakt of die meer dan zeven sessies ontvingen. De werknemers die de zeven sessies van het basisdeel ontvingen hadden ook het laagste aantal verzuimdagen na twaalf maanden follow-up. Echter, deze bevindingen willen nog niet automatisch zeggen dat het volgen van zeven sessies op zich tot een beter resultaat zal leiden. Bovendien was de power van deze studie laag, door de kleine aantallen van de subgroepen. Toekomstig onderzoek wordt aanbevolen om onderzoek te doen naar het optimale aantal sessies voor elke individuele deelnemer.

In de algemene discussie, hoofdstuk 7, worden de belangrijkste bevindingen van de voorgaande hoofdstukken beschreven en bediscussieerd in termen van methodologische kwaliteit, overwegingen voor implementatie van de preventieve strategie, implicaties voor de betrokken partijen, suggesties voor verdere verbetering van de preventieve benadering en aanbevelingen voor toekomstig onderzoek. Dit proefschrift laat zien dat de preventieve benadering een veelbelovende methode is. Het screeningsinstrument is in staat om werknemers met een hoog risico op toekomstig verzuim 
en met milde tot ernstige depressieve klachten te identificeren. De preventieve interventie blijkt effectief in het voorkomen van langdurig ziekteverzuim en het reduceren van depressieve klachten. Voor de implementatie van de preventieve benadering in de bedrijfsgezondheidszorg moeten een aantal aandachtspunten in ogenschouw worden genomen, zoals het feit dat het uitvoeren van de preventieve benadering een omvangrijk en tijdsintensief proces inhoudt, waarbij vele partijen betrokken zijn. Speciale aandacht dient besteed te worden aan het begeleiden van geselecteerde werknemers om deel te nemen aan de preventieve interventie. Uit het onderzoek is gebleken dat de motivatie om daadwerkelijk deel te gaan nemen beïnvloed kan worden door bijvoorbeeld het niet ervaren van gezondheidsklachten, hetgeen zich kan voordoen omdat de focus van het onderzoek gericht is op werknemers met relatief milde gezondheidsklachten. Voor zowel de werknemer, de werkgever, zorgverzekeringen en de samenleving is de verwachting dat zij baat zullen hebben bij het invoeren van de preventieve benadering. Desondanks blijven er nog genoeg vragen onbeantwoord. Toekomstig onderzoek zou zich onder andere kunnen richten op een gedetailleerde analyse van de kosteneffectiviteit van de preventieve benadering, als ook op de toepassing van de preventieve benadering in verschillende soorten bedrijven en sectoren en bij verschillende doelpopulaties. 


\section{Dankwoord}

Eindelijk is het dan zover, het boekje is af! In de afgelopen jaren hebben veel mensen een bijdrage geleverd aan de totstandkoming van dit proefschrift. Al deze mensen wil ik hartelijk bedanken. Daarnaast wil ik een aantal mensen in het bijzonder bedanken.

Allereerst wil ik mijn promotor en dagelijkse begeleider IJmert en mijn co-promotor en dagelijkse begeleidster Nicole van harte bedanken voor de fijne samenwerking. Ondanks dat het mijn proefschrift is geworden, heb ik altijd het gevoel gehad dat het ons project was. Bedankt dat ik altijd een beroep op jullie kon doen, hierdoor heb ik nooit het gevoel gehad er alleen voor te staan. IJmert, bedankt dat ik altijd bij je kon binnen vallen, tijdens het project hebben we voor veel keuzes en ad-hoc beslissingen gestaan die moesten worden opgelost of uitgevoerd, maar er was ook altijd tijd om te overleggen over allerlei andere onderwerpen. Bedankt ook voor je steun en de interesse in hoe het met mij persoonlijk ging. Nicole, bedankt voor je onaflatende betrokkenheid bij het project en het in de gaten houden van alle facetten van het onderzoek, je altijd tijdige en nauwkeurige beoordeling van de artikels en andere stukken, en de deur die altijd voor me open stond om te overleggen over werk maar ook over privé-aangelegenheden.

Ook wil ik mijn andere promotor Piet bedanken. Piet bedankt voor de altijd kritische vragen tijdens onze projectgroepvergaderingen, het bewaken van de tijdsplanning van het project en de kritische feedback op de artikelen. Daarnaast een woord van dank aan de twee overige leden van de Maastrichtse projectgroep, Ludovic en Marcus. Ludovic, bedankt voor je kritische feedback op de artikelen en voor de hulp bij de statistische analyses en het gebruiksklaar maken van de verzuim database. Marcus, bedankt voor je psychologische kijk op het onderzoek, de inspirerende manier waarop je de psychologen getraind hebt en mij gemotiveerd hebt om 'er iets moois van te maken'. Bedankt voor je hulp bij de opzet en het uitwerken van het artikel over 'protocol adherence' en je kritische feedback op de andere stukken.

Vervolgens wil ik Ate Berkouwer, Rudy van Leusden en Gladys Tjin A Ton van Arbodienst Beter, onze onderzoekspartner in het project, hartelijk bedanken voor de zeer plezierige samenwerking. Ate en Gladys bedankt voor het vertrouwen in het onderzoek, jullie enthousiasme tijdens onze vergaderingen, de altijd snelle beoordeling van artikelen en reacties op mijn vragen heb ik zeer gewaardeerd. Ik hoop dat de samenwerking met arbeidsepidemiologie nog lang mag blijven bestaan.

Verder wil ik nog een aantal mensen van Arbodienst Beter in het bijzonder bedanken die hebben meegewerkt om het onderzoek tot een succes te maken. Allereerst Bob Couwenberg, bedankt voor alle ondersteuning die ik mocht ontvangen, het aanleveren en gereed maken van de databestanden en voor de mutaties die jij nauwlettend in de gaten hield. Marja Binsbergen, bedankt voor alle 
administratieve ondersteuning en de doorverwijzing van de deelnemers van het onderzoek. Jolanda Doornekamp en Els Blom bedankt voor het doorpluizen van gegevens die we nodig hadden voor het onderzoek. De bedrijfsmaatschappelijk werkers, Dick de Bruijn, Susan Dijkstra, Wilmy ten Haaf, Hannelore Kalter en Pia Batting, wil ik bedanken voor hun inzet met betrekking tot de toegeleiding van de deelnemers naar de interventie. Dick, een speciaal woord van dank aan jou, voor het beantwoorden van al mijn vragen en je bijdrage tijdens de trainingsdagen voor de psychologen.

Int Pos van Cenzo B.V. wil ik bedanken voor de deelname aan het onderzoek en de fijne samenwerking. De psychologen die deelgenomen hebben aan het onderzoek, Jenny Biemans, Shanti Decoz, James Haasnoot, Annemart Janssen, Anneke Kuipers, Gerdie Langenhuizen, Michel Ooms, Gelske Oppewal, Marcella Udenhout en René Verhoog, wil ik bedanken voor het verzorgen van de interventie, hun inzet en bijdrage aan het onderzoek.

Ook wil ik de 'bouwers', Arnoud Arntz, Anja van den Hout, Theo Bouman en Marcus Huibers bedanken voor het ontwikkelen van het protocol en de werkboeken voor de interventie en tevens voor hun enthousiaste en inspirerende optreden tijdens de trainingsdagen voor de psychologen.

De medewerkers van ABN AMRO Bank die deelgenomen hebben aan het onderzoek wil ik bedanken voor het invullen van alle vragenlijsten en het meedoen aan de interventie. Zonder jullie deelname was er geen onderzoek!

Loes van Bokhoven, bedankt dat je als onafhankelijk arts wilde optreden in het onderzoek.

Fred Stevens wil ik bedanken voor zijn bijdrage aan het artikel over hulpzoekend gedrag. Het duurde enige tijd voordat we de juiste aanpak te pakken hadden; bedankt voor alle discussies en suggesties!

Jos Bruystens, het prachtige omslag van dit proefschrift heb ik aan jou te danken, als ook de layout. Daarnaast heb je ook de bijzonder mooie vragenlijsten, folders en posters voor het project ontworpen. Je hebt er iedere keer weer voor gezorgd dat alles op tijd en picobello in orde werd afgeleverd. Jos, hartstikke bedankt!

Dan een woord van dank aan mijn (oud-) collega's van Arbeidsepidemiologie. Jos, bedankt voor je veelvuldige ondersteuning bij het project, zoals het maken van de databases en het invoeren van de vragenlijsten, en ook voor alle computeraangelegenheden waarvoor ik altijd bij je terecht kon. Petra, bedankt voor je hulp bij het invoeren van alle vragenlijsten en het bijhouden van de administratie van het project. Saskia (mijn eerste kamergenote), Lore en Stephanie, jullie gingen mij voor als aio's bij Arbeid. Jullie waren al een heel eind onderweg met jullie eigen promotie-onderzoek toen ik begon. Ik was geruime tijd het 'jonkie' van onze groep en kon altijd bij jullie terecht, bedankt voor alle tips 
en adviezen. Ik denk met veel plezier terug aan onze gezellige, regelmatig terugkerende Thembidates. Karolien, mijn huidige 'roomy', bedankt voor je steun tijdens alle ups en downs behorende bij het onderzoek, voor alle gesprekken over werk maar ook over privé-zaken, maar vooral ook voor de gezelligheid en je plezierige gezelschap. De eindstreep komt ook voor jou al in zicht, succes met de laatste loodjes en ik ben benieuwd naar jouw boekje! Danielle, Nathalie, Carin en Karolina bedankt voor jullie steun, interesse en de fijne samenwerking.

Naast de collega's van Arbeid wil ik ook de overige collega's van epidemiologie bedanken voor hun collegialiteit en de gezelligheid. Nathalie en Yvonne, bedankt voor jullie hulp bij het plannen van afspraken en voor het beantwoorden van een heleboel vragen. Harry, bedankt voor je hulp bij het maken van de access-databases en de overige computergerelateerde vragen.

De (ex-)aio's van epidemiologie wil ik graag bedanken voor de interesse in mijn onderzoek en de steun, de gezellige lunches, wandelingen en (aio-)uitjes. Diegenen die nog niet gepromoveerd zijn wens ik veel succes met hun eigen promotie-onderzoek!

Familie, schoonfamilie, vrienden en bekenden bedank ik voor hun interesse in mijn werk en hun steun. Daarbij wil ik Hylke in het bijzonder bedanken, voor je onaflatende interesse in hoe het met mijn proefschrift en met mij gaat. Keer op keer heb je mijn verhaal met alle geduld van de wereld aangehoord en me van advies voorzien. Dank je wel daarvoor. Ik ben dan ook heel blij dat je als paranimf naast mij wil staan! Anneke, ooit tegelijk begonnen, maar jij bent inmiddels al ruim een jaar gepromoveerd! Bedankt voor je interesse in mijn onderzoek, de gezellige theepauzes en lunchwandelingetjes. Het zou leuk zijn als we in de toekomst nog eens kunnen samenwerken!

Lieve pap en mam, bedankt voor jullie interesse in mijn werk, jullie steun en vertrouwen. Jullie staan altijd klaar voor Chris en mij. Na vijf jaar kunnen jullie nu eindelijk zien waar ik al die tijd aan gewerkt heb. Lieve Chris, mijn grote kleine broer, naar jou kijk ik op. Ik ben er trots op dat je tijdens mijn promotie naast mij staat en ik kijk er naar uit om over een tijdje jouw proefschrift te mogen ontvangen! Lieve Luuk, bedankt voor je steun en je luisterend oor, maar vooral ook voor je relativeringsvermogen. Jij kunt alles zo heerlijk luchtig maken! Je staat bij mijn promotie dan wel niet naast mij, maar ik weet dat je er altijd voor mij bent en dat je achter mij staat.

Monique

Maastricht, maart 2011 



\section{About the author}

Monique Lexis was born on May 9, 1981 in Maastricht, the Netherlands. After completing secondary school (VWO) at the Stella Maris College in Meerssen in 1999, she studied Occupational Therapy at Hogeschool Zuyd in Heerlen and graduated in 2003. Additionally, she studied Health Sciences at Maastricht University which resulted in a master's degree in Health Sciences, specialization Movement Sciences, in 2005 (cum laude). From January 2006 until October 2010, she worked as a PhD student at the department of Epidemiology of Maastricht University (CAPHRI School for Public Health and Primary Care, Maastricht University), on a research project that aimed to examine the efficacy of a preventive strategy on the prevention of long-term sickness absence and major depression, among employees who are identified at high risk of future sickness absence and with mild to severe depressive complaints. The project was funded by the Netherlands Organization for Health Research and Development (Nederlandse organisatie voor gezondheidszorgonderzoek en zorginnovatie, ZonMw), and resulted in the present thesis. During her period as a PhD student she completed a master's degree in Epidemiology. Monique currently works at the Clinical Trial Center Maastricht (CTCM) on a project that aims to develop a prospective risk classification for clinical studies. 



\section{List of publications}

\section{Peer reviewed manuscripts}

Lexis MAS, Jansen NWH, van Amelsvoort LGPM, van den Brandt PA, Kant IJ. Depressive complaints as a predictor of sickness absence among the working population. Journal of Occupational and Environmental Medicine 2009;51:887-95.

Vluggen TPMM, Lexis MAS, Schuurman JJE, Schols JMGA. Effectiviteit van functionele oefentherapie in vergelijking met weerstandstraining op de uitvoering van adl-activiteiten en de spierkracht bij thuiswonende ouderen: een systematische literatuurstudie. Nederlands Tijdschrift voor Fysiotherapie 2009;119:122-8.

Lexis MAS, Jansen NWH, Stevens FCJ, van Amelsvoort LGPM, Kant IJ. Experience of health complaints and help seeking behavior in employees screened for depressive complaints and risk of future sickness absence. Journal of Occupational Rehabilitation 2010;20(4):537-46.

Lexis CP, Rahel BM, van Langen $\mathrm{H}$, Lexis MA, Hoffland GA, Post PJ, Meeder JG. Cardiac magnetic resonance imaging in daily practice in a peripheral medical centre: description of the first 383 patients. Netherlands Heart Journal 2010;18(11):524-30.

Lexis MAS, Jansen NWH, Huibers MJH, van Amelsvoort LGPM, Berkouwer A, Tjin A Ton G, van den Brandt PA, Kant IJ. Prevention of long-term sickness absence and major depression in high risk employees: a randomized controlled trial. Occupational and Environmental Medicine; in press.

Lexis CP, van der Horst IC, Rahel BM, Lexis MA, Kampinga MA, Gu YL, de Smet BJ, Zijlstra F. Impact of chronic total occlusions on markers of reperfusion, infarct size, and long-term mortality: A substudy from the TAPAS-trial. Catheterization and Cardiovascular Interventions; in press.

\section{Submitted manuscripts}

Lexis MAS, Jansen NWH, van Amelsvoort LGPM, Huibers MJH, Berkouwer A, Tjin A Ton G, van den Brandt $P A$, Kant IJ. Prediction of long-term sickness absence among employees with depressive complaints.

Lexis MAS, Jansen NWH, Huibers MJH, van Amelsvoort LGPM, Berkouwer A, Tjin A Ton G, van den Brandt PA, Kant IJ. The relationship between protocol adherence and the efficacy of a preventive intervention aimed at the prevention of long-term sickness absence and major depression. 


